\title{
On The Center Problem For Ordinary Differential Equations
}

\author{
Alexander Brudnyi* \\ Department of Mathematics and Statistics \\ University of Calgary, Calgary \\ Canada
}

\begin{abstract}
The classical Center-Focus problem posed by H. Poincaré in 1880's asks about the characterization of planar polynomial vector fields such that all their integral trajectories are closed curves whose interiors contain a fixed point, a center. In this paper we describe a new general approach to the Center Problem.
\end{abstract}

\section{Contents}

1. Introduction. 2

2. Monodromy in the Center Problem. 6

3. Algebraic Model for the Center Problem. 13

4. Proofs of Results of Section 2.1. 17

5. Proofs of Results of Sections 2.2 and 2.3. 28

$\begin{array}{lll}\text { 6. Proof of Theorem 1.9. } & 28\end{array}$

7. Proofs of Theorem 2.12 and Corollaries 2.10, 2.15 and 2.17. 32

8. Proofs of Theorems 2.18, 2.20 and Corollaries 2.21 and 2.22. 37

9. Proofs of Results of Section 3.1. 38

10. Proofs of Results of Section 3.2. 45

11. Proofs of Results of Section 3.3. 50

${ }^{*}$ Research supported in part by NSERC.

2000 Mathematics Subject Classification. Primary 34C07, Secondary 32E20, 58K10.

Key words and phrases. Center Problem, Lipschitz function, monodromy, iterated integrals. 


\section{Introduction.}

1.1. Let $L^{\infty}\left(S^{1}\right)$ be the Banach space of bounded complex-valued functions on the unit circle $S^{1}$. We will also identify elements of $L^{\infty}\left(S^{1}\right)$ with bounded $2 \pi$-periodic functions on $\mathbb{R}$. Let us consider the ordinary differential equation

$$
\frac{d v}{d x}=\sum_{i=1}^{\infty} a_{i}(x) v^{i+1}
$$

where all $a_{i} \in L^{\infty}\left(S^{1}\right)$. If the coefficients of (1.1) grow not very fast for a sufficiently small initial value one can solve this equation by the Picard iteration method so that the (generalized) solution is a Lipschitz function on $[0,2 \pi]$. (I. e., in this way we obtain a function $v$ for which (1.1) holds almost everywhere on $[0,2 \pi]$.) Moreover, there is a unique solution with the prescribed initial value $v(0)$. We say that equation (1.1) determines a center if for any sufficiently small initial values $v(0)$ the solution of (1.1) satisfies $v(0)=v(2 \pi)$. The Center Problem for equation (1.1) is to find conditions on the coefficients $a_{i}$ under which this equation determines a center. This problem arises naturally in the framework of the qualitative theory of ordinary differential equations created by $\mathrm{H}$. Poincaré. The main purpose of the theory can be described as follows: without explicitly solving a given differential equation, using only certain properties of its right-hand side, to try and give an as complete as possible description of the geometry of the solution curves of this equation (where they are defined). In this paper we present a new general approach to the Center Problem for equation (1.1).

1.2. The Center Problem for (1.1) is closely related to the classical Center-Focus problem first studied by Poincaré [P] and further developed by Lyapunov [L], Bendixson $[\mathrm{B}]$ and Frommer $[\mathrm{F}]$. In this case one considers the system of ODEs in the plane

$$
\frac{d x}{d t}=X(x, y), \quad \frac{d y}{d t}=Y(x, y)
$$

where $X, Y$ are real polynomials of degree $d$, or more generally, real analytic functions defined in an open neighbourhood of the origin $P:=(0,0) \in \mathbb{R}^{2}$. One assumes that $P$ is an equilibrium point, i.e. $X(0,0)=Y(0,0)=0$. The equilibrium point is called a center if there exists an open neighbourhood $U$ of $P$ that does not contain another equilibrium points such that any trajectory of the vector field (1.2) that intersects $U \backslash\{P\}$ in some point is closed. Suppose

$$
X(x, y)=a x+b y+\sum_{k=2}^{\infty} X_{k}(x, y), \quad Y(x, y)=c x+d y+\sum_{k=2}^{\infty} Y_{k}(x, y)
$$

where $X_{k}, Y_{k}$ are real homogeneous polynomials of degree $k$. In the aforementioned papers the case of a non-degenerated equilibrium point was studied, i.e. the matrix

$$
A=\left(\begin{array}{ll}
a & b \\
c & d
\end{array}\right)
$$

assumed to be invertible. It was proved by Poincaré that a necessary condition for $P$ to be a center is that $A$ has pure imaginary eigenvalues. In this case, making a 
linear change of variables in (1.2) and then a linear reparametrization of trajectories one reduces (1.2) to an equivalent system:

$$
\frac{d x}{d t}=-y+F(x, y), \quad \frac{d y}{d t}=x+G(x, y)
$$

where $F, G$ are real analytic functions in an open neighbourhood of $P$ whose Taylor expansions at $P$ do not contain constant and linear terms. For $F, G$ polynomials of a given degree, the classical Poincaré Center-Focus Problem ${ }^{1}$ asks about conditions on the coefficients of $F$ and $G$ under which all trajectories of (1.3) situated in a small open neighbourhood of the origin are closed. (A similar problem can be posed for the general case.) Poincaré proved that $P$ is a center if and only if the coefficients of $F$ and $G$ satisfy a certain infinite system of algebraic equations $E_{1}, E_{2}, \ldots$ such that the coefficients of $E_{n}$ are functions in the solutions of $E_{1}, \ldots, E_{n-1}$. Thus in order to solve $E_{n}$ one first should solve all the previous equations. In the present paper we give another completely new characterization of centers in terms of an infinite system of algebraic equations $\widetilde{E}_{1}, \widetilde{E}_{2}, \ldots$ such that each $\widetilde{E}_{n}$ can be solved independently of the others (see Theorem 2.2). This allows to establish non-existence of a center using only one successfully chosen equation $\widetilde{E}_{n}$. Unfortunately, trying to apply either this or Poincaré criterion in the converse direction, gives rise, in the general case, to almost insurmountable difficulties. Therefore, as it was emphasized by Poincaré, it is the matter of great importance to find typical situations for which all equations of the system determining centers are satisfied. As an example, Poincaré described a case related to certain symmetries for $F$ and $G$. Some results of our paper can be viewed as a further development in this direction (see Sections 2.4 and 2.5).

Another necessary and sufficient condition for $P$ to be a center in (1.3) was obtained by Lyapunov [L]. His result says that $P$ is a center if and only if system (1.3) has an independent from $t$ real analytic integral $F(x, y)=C$. Unfortunately, trying to apply the Lyapunov condition gives rise, in general, to difficulties comparable with those for Poincaré's type criterions.

It is important to observe that passing to polar coordinates in (1.3) we rewrite this in the form

$$
\frac{d r}{d \theta}=\frac{P}{1+Q} r
$$

where $P(r, \phi):=\frac{x F(x, y)+y G(x, y)}{r^{2}}, Q(r, \phi):=\frac{x G(x, y)-y F(x, y)}{r^{2}},(x=r \cos \phi, y=r \sin \phi)$. If the moduli of the coefficients of $F$ and $G$ are small enough we can expand the right-hand side of (1.4) as a series in $r$ to obtain an equation of type (1.1) whose coefficients are trigonometric polynomials. This reduces the Center Problem for (1.3) to the Center Problem for a class of equations (1.1) whose coefficients depend polynomially on the coefficients of (1.3).

1.3. Let us briefly discuss the content of the present paper.

Let $X_{i}:=L^{\infty}\left(S^{1}\right)$ be the space of all coefficients $a_{i}$ from (1.1), and $X$ be the

\footnotetext{
${ }^{1}$ This name originates from the fact that in the case of system (1.3) the point $P$ can be either a center or a focus.
} 
complex vector space of sequences $a=\left(a_{1}, a_{2}, \ldots\right) \in \prod_{i \geq 1} X_{i}$ satisfying

$$
\sup _{x \in S^{1}}\left|a_{i}(x)\right| \leq l^{i}, \quad i=1,2, \ldots,
$$

for some positive $l$ (depending on $a$ ). From Picard iteration it follows that for any $a \in X$ the corresponding equation (1.1) is locally solvable for sufficiently small initial values. Let $\mathcal{C} \subset X$ be the center set of equation (1.1), that is, the set of those $a \in X$ for which the corresponding equations (1.1) determine centers. One of our main results (see Theorem 2.1) shows that $a \in \mathcal{C}$ if and only if the monodromy of a certain linear differential equation $\frac{d F}{d x}=A F$ defined on $S^{1}$ is trivial. Here $A$ is a function on the circle with values in the associative algebra $\mathcal{A}\left(S_{1}, S_{2}\right)[[t]]$ of formal power series in $t$ whose coefficients are complex non-commutative polynomials in variables $S_{1}$ and $S_{2}$ satisfying the relation

$$
\left[S_{1}, S_{2}\right]:=S_{1} S_{2}-S_{2} S_{1}=-S_{2}^{2} .
$$

Next, let us consider the iterated integrals

$$
I_{i_{1}, \ldots, i_{k}}(a):=\int \cdots \int_{0 \leq s_{1} \leq \cdots \leq s_{k} \leq 2 \pi} a_{i_{k}}\left(s_{k}\right) \cdots a_{i_{1}}\left(s_{1}\right) d s_{k} \cdots d s_{1}
$$

defined on $X$ (for $k=0$ we assume that this equals 1 ). They can be thought of as $k$ linear holomorphic functions on $X$. By the Ree formula $[\mathrm{R}]$ (see also our Section 6.3) the linear space generated by all such functions is an algebra. A linear combination of iterated integrals of order $\leq k$ is called an iterated polynomial of degree $k$.

As an important corollary of Theorem 2.1 we obtain an explicit description of the center set $\mathcal{C}$ as a subset of $X$ determined by a system of polynomial equations $c_{n}(a)=0, n=1,2, \ldots$, where

$$
\begin{gathered}
c_{n}(a)=\sum_{i_{1}+\ldots+i_{k}=n} c_{i_{1}, \ldots, i_{k}} I_{i_{1}, \ldots, i_{k}}(a), \quad \text { and } \\
c_{i_{1}, \ldots, i_{k}}=\left(n-i_{1}+1\right) \cdot\left(n-i_{1}-i_{2}+1\right) \cdot\left(n-i_{1}-i_{2}-i_{3}+1\right) \cdots 1 .
\end{gathered}
$$

On the other hand, we present a natural "parametrization" of the center set (see Theorem 2.4). This makes it possible to construct elements from $\mathcal{C}$ explicitly.

We say that equation (1.1) corresponding to $a \in X$ determines a universal center, if $I_{i_{1}, \ldots, i_{k}}(a)=0$ for all positive integers $i_{1}, \ldots, i_{k}$ and $k \geq 1$. The set $\mathcal{U}$ of universal centers is, in a sense, a stable part of the center set $\mathcal{C}$. In our paper we describe some classes of equations (1.1) which determine universal centers. Some of our results reveal a connection with the so-called composition condition whose role and importance for the Center Problem was studied in [Y], [BFY1], [BFY2], [AL] for the special case of Abel differential equations (cf. Corollary 2.17 below).

Next, we focus our attention on the most important algebraic aspects of the Center Problem that were not previously known in this area.

To this end we introduce a natural multiplication $*: X \times X \rightarrow X$ of elements of $X$ similar to the multiplication of continuous paths in the Homotopy Theory (see Section 3.1). We say that $a, b \in X$ are equivalent (written, $a \sim b$ ) if $a * b^{-1} \in \mathcal{U}$. In 
fact, we will show then that $\sim$ is an equivalence relation, that is $X$ partitions into mutually disjoint equivalence classes. Throughout this paper, the set of all these classes will be denoted by $G(X)$. An important observation is that the multiplication * induces a similar multiplication $\cdot: G(X) \times G(X) \rightarrow G(X)$ such that the pair $(G(X), \cdot)$ is a group. Moreover, we will prove that the iterated integrals are constant on any equivalence class. Thus without loss of generality they may be considered as functions on $G(X)$. Also, it could be extracted from the construction of $G(X)$ that these functions separate points on $G(X)$.

Further, let us provide $G(X)$ with the weakest topology $\tau$ in which all iterated integrals (considered as functions on $G(X)$ ) are continuous. One of the central results of Section 3.1 says that $(G(X), \cdot, \tau)$ is a separable topological group satisfying the following properties

(1) $(G(X), \tau)$ is contractible to a point, arcwise connected, locally arcwise and simply connected;

(2) $G(X)$ is residually torsion free nilpotent (that is, the set of all finite-dimensional unipotent representations separates elements of $G(X))$.

To formulate our next result we introduce the set $G_{c}[[r]]$ of complex power series of the form $f(r)=r+\sum_{i=1}^{\infty} d_{i} r^{i+1}$ each convergent in some open neighbourhood of $0 \in \mathbb{C}$. Consider the functions $d_{i}: G_{c}[[r]] \rightarrow \mathbb{C}$ such that $d_{i}(f)$ equals the $i+1$-st coefficient of the Taylor expansion of $f$ at 0 . Let $\tau^{\prime}$ be the weakest topology on $G_{c}[[r]]$ in which all the functions $d_{i}$ are continuous. Also, we consider the multiplication on $G_{c}[[r]]$ defined by the composition of series. Then, as in the case of $G(X)$, we will prove that $\left(G_{c}[[r]], \circ, \tau^{\prime}\right)$ is a separable topological group satisfying the properties

(3) $\left(G_{c}[[r]], \tau^{\prime}\right)$ is contractible to a point, arcwise connected, locally arcwise and simply connected;

(4) $G_{c}[[r]]$ is residually torsion free nilpotent.

Now, for any $a \in X$ by $v(x ; r ; a), x \in[0,2 \pi]$, we denote the Lipschitz solution of equation (1.1) corresponding to $a$ with initial value $v(0 ; r ; a)=r$. It is clear that for every $x$ we have $v(x ; r ; a) \in G_{c}[[r]]$. The function $P(a)(r):=v(2 \pi ; r ; a)$ is called the first return map of equation (1.1). In Section 3.2 we will show that

$$
P(a * b)=P(b) \circ P(a) .
$$

This fundamental property together with the fact that $P(a)(r) \equiv r$ for any $a \in \mathcal{U}$ imply that there exists a map $\widehat{P}: G(X) \rightarrow G_{c}[[r]]$ such that $\widehat{P}([a]):=P(a)$ where $[a]$ denotes the equivalence class containing $a \in X$. Then we will prove that

(5) $\widehat{P}$ is a surjective homomorphism of topological groups;

(6) The kernel $\widehat{\mathcal{C}} \subset G(X)$ of $\widehat{P}$ coincides with the image of the center set $\mathcal{C} \subset X$ in $G(X)$; 
(7) $(\widehat{\mathcal{C}}, \tau)$ is contractible to a point, arcwise connected, locally arcwise and simply connected.

Let us consider the quotient group $Q(X)=G(X) / \widehat{\mathcal{C}}$. By $\pi: G(X) \rightarrow Q(X)$ we denote the quotient homomorphism. It follows from the preceding discussion that every function $c_{i}$ in the definition of the center set $\mathcal{C}$ satisfies $c_{i}(a)=c_{i}(b)$ for $a \sim b$. Therefore every $c_{i}$ can be considered as a continuous function on $G(X)$. In fact, we will show that these functions are constant on any fibre of the map $\pi$ and hence they determine functions $\bar{c}_{i}: Q(X) \rightarrow \mathbb{C}$. Let $\tau^{\prime \prime}$ be the weakest topology on $Q(X)$ in which all the functions $\bar{c}_{i}$ are continuous. Then we will prove that

(8) $\left(Q(X), \tau^{\prime \prime}\right)$ is a topological group.

(9) The homomorphism $\widehat{P}$ determines an isomorphism $\bar{P}: Q(X) \rightarrow G_{c}[[r]]$ of topological groups defined by $\widehat{P}=\bar{P} \circ \pi$.

Also, we will show that there is a continuous map $T: G_{c}[[r]] \rightarrow G(X)$ such that $\widehat{P} \circ T=i d$. In particular, we obtain that

(10) The map $\widetilde{T}: G_{c}[[r]] \times \widehat{\mathcal{C}} \rightarrow G(X), \widetilde{T}(f, g):=T(f) \cdot g$, is a homeomorphism.

Finally, in Section 3.3 we study some algebraic properties of the group $G(X)$ and its specific subgroups defined over certain subfields of the filed $\mathbb{C}$.

Acknowledgment. I would like to thank Professor Y. Yomdin for very fruitful discussions during his visit to Calgary. I also would like to thank Professors L. Bos and Y. Brudnyi for the valuable help and the support of this work.

The next two sections contain a more leisurely description of the main results of the paper.

\section{Monodromy in the Center Problem.}

2.1. Our first result shows that the Center Problem for (1.1) is equivalent to the triviality of the monodromy of a certain linear system of ODEs.

In order to formulate this result one introduces the associative algebra $\mathcal{A}\left(S_{1}, S_{2}\right)$ with unit $I$ of non-commutative polynomials with complex coefficients in variables $S_{1}$ and $S_{2}$ satisfying the relation

$$
\left[S_{1}, S_{2}\right]:=S_{1} S_{2}-S_{2} S_{1}=-S_{2}^{2} .
$$

Then $\mathcal{A}\left(S_{1}, S_{2}\right)[[t]]$ stands for the associative algebra of formal power series in $t$ whose coefficients are elements from $\mathcal{A}\left(S_{1}, S_{2}\right)$. For an element $a=\left(a_{1}, a_{2}, \ldots\right) \in X$ (see Section 1.3) let us consider the equation on $S^{1}$

$$
\frac{d F}{d x}=\left(\sum_{i=1}^{\infty} a_{i}(x) t^{i} S_{1} S_{2}^{i-1}\right) F .
$$

This can be solved locally by the Picard iteration method to obtain a local solution $F(x)$ as a function in $x$ with values in the group $G\left(S_{1}, S_{2}\right)[[t]]$ of invertible elements 
of $\mathcal{A}\left(S_{1}, S_{2}\right)[[t]]$ whose coefficients are Lipschitz. As usual, the monodromy of $(2.2)$ is a homomorphism $\rho: \mathbb{Z} \rightarrow G\left(S_{1}, S_{2}\right)[[t]]$ where $\mathbb{Z}$ is the fundamental group of the unit circle $S^{1}$. It is the only obstruction to extending local solutions of (2.2) to global ones.

Theorem 2.1 Equation (1.1) determines a center if and only if the monodromy of the corresponding equation (2.2) is trivial.

From this result we obtain the following characterization of centers for (1.1). As before, let $X$ be the complex vector space of sequences $a=\left(a_{1}, a_{2}, \ldots\right)$ satisfying (1.5). The functions of the form

$$
I_{i_{1}, \ldots, i_{k}}(a):=\int \cdots \int_{0 \leq s_{1} \leq \cdots \leq s_{k} \leq 2 \pi} a_{i_{k}}\left(s_{k}\right) \cdots a_{i_{1}}\left(s_{1}\right) d s_{k} \cdots d s_{1}
$$

will be called the basic iterated integrals on $X$.

Now, for a sufficiently small $r$, let $v(x ; r ; a), x \in[0,2 \pi]$, be the Lipschitz solution of equation (1.1) corresponding to $a \in X$ with initial value $v(0 ; r ; a)=r$. Then $P(a)(r):=v(2 \pi ; r ; a)$ is the first return map of this equation.

Theorem 2.2 For sufficiently small initial values $r$ the first return map $P(a)$ is an absolutely convergent power series $P(a)(r)=r+\sum_{n=1}^{\infty} c_{n}(a) r^{n+1}$, where

$$
\begin{gathered}
c_{n}(a)=\sum_{i_{1}+\ldots+i_{k}=n} c_{i_{1}, \ldots, i_{k}} I_{i_{1}, \ldots, i_{k}}(a), \quad \text { and } \\
c_{i_{1}, \ldots, i_{k}}=\left(n-i_{1}+1\right) \cdot\left(n-i_{1}-i_{2}+1\right) \cdot\left(n-i_{1}-i_{2}-i_{3}+1\right) \cdots 1 .
\end{gathered}
$$

The center set $\mathcal{C} \subset X$ of equation (1.1) is determined by the system of polynomial equations $c_{n}(a)=0, n=1,2, \ldots$.

Corollary 2.3 (a) $\quad c_{n}(a)=I_{n}(a)+f_{n}(a)$ where $I_{n}(a):=\int_{0}^{2 \pi} a_{n}(s)$ ds and $f_{n}$ is an iterated polynomial of degree $n$ in $a_{1}, \ldots, a_{n-1}$;

(b) The set

$$
\mathcal{C}_{n}=\left\{a \in X: c_{1}(a)=c_{2}(a)=\ldots=c_{n}(a)=0\right\}
$$

is a closed complex submanifold of $X$ of codimension $n$ containing $0 \in X$;

(c) The tangent space to $\mathcal{C}_{n}$ at 0 is determined by equations $I_{1}(a)=\ldots=I_{n}(a)=0$.

Another characterization of centers of (1.1) is given by the following theorem.

Theorem 2.4 An element $a=\left(a_{1}, a_{2}, \ldots\right) \in X$ belongs to the center set $\mathcal{C}$ if and only if there is a sequence $u_{1}, u_{2}, \ldots$ of $2 \pi$-periodic Lipschitz functions such that $u_{i}(0)=0$ for any $i$ and

$$
\sum_{i=1}^{\infty} a_{i}(x) t^{i+1}=-\frac{\sum_{k=1}^{\infty} u_{k}^{\prime}(x) t^{k+1}}{1+\sum_{k=1}^{\infty}(k+1) u_{k}(x) t^{k}}
$$

as formal power series in $t$. 
This result gives a "parametrization" of the center set $\mathcal{C} \subset X$ but leaves open the question on existence of such a sequence $u_{1}, u_{2} \ldots$ for a specific $a \in X$.

2.2. Based on Theorem 2.1 we split the Center Problem for (1.1) into two parts.

To this end we introduce the associative algebra $\mathcal{A}\left(X_{1}, X_{2}\right)$ with unit $I$ of noncommutative polynomials with complex coefficients in free non-commutative variables $X_{1}$ and $X_{2}$. By $\mathcal{A}\left(X_{1}, X_{2}\right)[[t]]$ we denote the associative algebra of formal power series in $t$ whose coefficients are elements from $\mathcal{A}\left(X_{1}, X_{2}\right)$. Then there is a natural homomorphism $\phi: \mathcal{A}\left(X_{1}, X_{2}\right)[[t]] \rightarrow \mathcal{A}\left(S_{1}, S_{2}\right)[[t]]$ uniquely defined by the relations $\phi\left(X_{1}\right)=S_{1}$ and $\phi\left(X_{2}\right)=S_{2}$. Next, for $a=\left(a_{1}, a_{2}, \ldots\right) \in X$ as in (2.2) let us consider the equation on the circle $S^{1}$

$$
\frac{d F}{d x}=\left(\sum_{i=1}^{\infty} a_{i}(x) t^{i} X_{1} X_{2}^{i-1}\right) F .
$$

Again one can solve this equation locally by Picard iteration. Then the monodromy $\widetilde{\rho}: \mathbb{Z} \rightarrow G\left(X_{1}, X_{2}\right)[[t]]$ of $(2.3)$ is the homomorphism of the fundamental group of $S^{1}$ into the group $G\left(X_{1}, X_{2}\right)[[t]]$ of invertible elements of $\mathcal{A}\left(X_{1}, X_{2}\right)[[t]]$. It is clear that

$$
\rho=\phi \circ \widetilde{\rho}
$$

Then from Theorem 2.1 follows

Corollary 2.5 If the monodromy $\widetilde{\rho}$ is trivial, then the corresponding equation (1.1) determines a center.

We will say that equation (1.1) determines a universal center, if the monodromy of the corresponding equation (2.3) is trivial. The set $\mathcal{U}$ of universal centers is, in a sense, a stable part of the center set $\mathcal{C}$. As we will see, $\mathcal{U} \neq \mathcal{C}$, in general. The next two sections describe some classes of equations (1.1) which determine universal centers.

2.3. Let $\omega(x):=\sum_{i=1}^{\infty} a_{i}(x) t^{i} X_{1} X_{2}^{i-1}$. As before we identify functions on the circle with $2 \pi$-periodic functions on $\mathbb{R}$. Recall that the fundamental solution of (2.3) is a $\operatorname{map} F: \mathbb{R} \rightarrow G\left(X_{1}, X_{2}\right)[[t]]$ such that $F(0)=I$ and $F^{\prime}(x)=\omega(x) \cdot F(x)$. It can be presented by Picard iteration (cf. $[\mathrm{Na}])$ in the form

$$
F(x):=I+\sum_{k=1}^{\infty} \int \cdots \int_{0 \leq s_{1} \leq \cdots \leq s_{k} \leq x} \omega\left(s_{k}\right) \cdots \omega\left(s_{1}\right) d s_{k} \cdots d s_{1}
$$

Also, it is easy to see that

$$
F(x)=\sum_{i=0}^{\infty} f_{i}\left(x ; X_{1}, X_{2}\right) t^{i}
$$

where $f_{0}=I$ and the other $f_{i}$ are homogeneous polynomials of degree $i$ in $X_{1}$ and $X_{2}$ whose coefficients are locally Lipschitz functions in $x \in \mathbb{R}$. Then the monodromy of $(2.3)$ is defined as $\widetilde{\rho}(n):=F(2 \pi n)=F(2 \pi)^{n}, n \in \mathbb{Z}$. 
Proposition 2.6 The monodromy of (2.3) is trivial, i.e. $F(2 \pi)=I$, if and only if for all positive integers $i_{1}, \ldots, i_{k}$ and $k \geq 1$

$$
\int \cdots \int_{0 \leq s_{1} \leq \cdots \leq s_{k} \leq 2 \pi} a_{i_{k}}\left(s_{k}\right) \cdots a_{i_{1}}\left(s_{1}\right) d s_{k} \cdots d s_{1}=0
$$

In particular then,

$$
\widetilde{a}_{i}(x):=\int_{0}^{x} a_{i}(s) d s, \quad i=1,2, \ldots,
$$

are $2 \pi$-periodic Lipschitz functions.

Next, let us consider equations

$$
F^{\prime}(x)=\omega_{n}(x) \cdot F(x) \quad \text { with } \quad \omega_{n}:=\sum_{i=1}^{n} a_{i}(x) t^{i} X_{1} X_{2}^{i-1}
$$

By $\widetilde{\rho}_{n}: \mathbb{Z} \rightarrow G\left(X_{1}, X_{2}\right)[[t]]$ we denote the monodromy of $(2.8)$.

Proposition 2.7 The monodromy of (2.3) is trivial if and only if all $\widetilde{\rho}_{n}$ are trivial. Moreover, the triviality of $\tilde{\rho}_{n}$ is equivalent to the fulfilment of equations (2.7) for any integers $1 \leq i_{1}, \ldots, i_{k} \leq n$, and any $k$.

2.4. Let us introduce the Lipschitz map $A_{n}: S^{1} \rightarrow \mathbb{C}^{n}, A_{n}(x)=\left(\widetilde{a}_{1}(x), \ldots, \widetilde{a}_{n}(x)\right)$, and set $\Gamma_{n}:=A_{n}\left(S_{1}\right)$. We require

Definition 2.8 The polynomially convex hull $\widehat{K}$ of a compact set $K \subset \mathbb{C}^{n}$ is the set of points $z \in \mathbb{C}^{n}$ such that if $p$ is any holomorphic polynomial in $n$ variables

$$
|p(z)| \leq \max _{x \in K}|p(x)|
$$

It is well known (see e.g. $[\mathrm{AW}]$ ) that $\widehat{K}$ is compact, and if $K$ is connected then $\widehat{K}$ is connected.

Theorem 2.9 If the monodromy $\widetilde{\rho}_{n}$ is trivial then for any domain $U$ containing $\widehat{\Gamma}_{n}$ the path $A_{n}: S^{1} \rightarrow U$ is contractible in $U$ to a point.

Since $A_{n}$ is Lipschitz, $\Gamma_{n}$ is of a finite linear measure. Then according to the remarkable result of Alexander $[\mathrm{A}], \widehat{\Gamma}_{n} \backslash \Gamma_{n}$ is a (possibly empty) pure one-dimensional analytic subset of $\mathbb{C}^{n} \backslash \Gamma_{n}$. In particular, since the covering dimension of $\Gamma_{n}$ is 1 , the covering dimension of $\widehat{\Gamma}_{n}$ is 2 . However, in general we do not know how to use this to get from Theorem 2.9 more information about $\Gamma_{n}$. Thus we restrict our presentation to several special cases.

Corollary 2.10 Suppose $\Gamma_{n}$ is triangulable and $\widehat{\Gamma}_{n}=\Gamma_{n}$. If the monodromy $\widetilde{\rho}_{n}$ is trivial then the path $A_{n}: S^{1} \rightarrow \Gamma_{n}$ is contractible inside $\Gamma_{n}$ to a point. Moreover, the contractibility of $A_{n}$ is equivalent to the factorization $A_{n}=A_{1 n} \circ A_{2 n}$ where $A_{2 n}: S^{1} \rightarrow G_{n}$ is a continuous map into a finite tree $G_{n} \subset \mathbb{R}^{N_{n}}$, and $A_{1 n}: G_{n} \rightarrow \Gamma_{n}$ is a finite continuous map. 
The converse result requires a much stronger than just triangulability condition on $\Gamma_{n}$ that is described by

Definition 2.11 A compact curve $C \subset \mathbb{R}^{N}$ is called Lipschitz triangulable if

(a) $C=\cup_{j=1}^{s} C_{i}$ and for $i \neq j$ the intersection $C_{i} \cap C_{j}$ consists of at most one point;

(b) There are Lipschitz embeddings $f_{i}:[0,1] \rightarrow \mathbb{R}^{N}$ such that $f_{i}([0,1])=C_{i}$;

(c) The inverse maps $f_{i}^{-1}: C_{i} \rightarrow \mathbb{R}$ are locally Lipschitz on $C_{i} \backslash\left(f_{i}(0) \cup f_{i}(1)\right)$.

Theorem 2.12 Suppose $\Gamma_{n}$ is Lipschitz triangulable, $\widehat{\Gamma}_{n}=\Gamma_{n}$, and $A_{n}: S^{1} \rightarrow \Gamma_{n}$ is contractible inside $\Gamma_{n}$ to a point. Suppose also that $A_{n}^{-1}(x)$ is countable for any $x \in \Gamma_{n}$. Then the corresponding monodromy $\widetilde{\rho}_{n}$ is trivial.

Remark 2.13 We will see from the proof that under the hypotheses of Theorem 2.12 the map $A_{2 n}: S^{1} \rightarrow \mathbb{R}^{N_{n}}$ is Lipschitz, $G_{n}$ is Lipschitz triangulable, and $A_{1 n}$ : $G_{n} \rightarrow \Gamma_{n}$ is locally Lipschitz outside a finite set.

Example 2.14 (1) If $A_{n}: S^{1} \rightarrow \mathbb{C}^{n}$ is non-constant analytic, then $\Gamma_{n}$ is Lipschitz triangulable, and $A_{n}^{-1}(x)$ is finite for any $x \in \Gamma_{n}$;

(2) To satisfy the second hypothesis of Corollary 2.10, one can suppose, e.g., that $\Gamma_{n}$ belongs to a compact $K_{n}$ in a $C^{1}$-smooth manifold $M_{n}$ with no complex tangents such that $\widehat{K}_{n}=K_{n}$. For instance, one can take any compact $K_{n}$ in $M_{n}=\mathbb{R}^{n}$ (for the proof see e.g. [AW, Th.17.1]).

Next, we consider maps into one-dimensional complex spaces. Suppose that one of the following two conditions is satisfied:

(1) $\Gamma_{n} \subset X$ where $X$ is a closed one-dimensional complex analytic subset of a domain $U \subset \mathbb{C}^{n}$ such that $U=\cup_{j} K_{j}$ with $K_{j} \subset \subset K_{j+1}$, and $\widehat{K}_{j}=K_{j}$ for any $j$;

(2) $\Gamma_{n} \subset X$ where $X \subset \mathbb{C}^{n}$ is a connected one-dimensional complex space with $\operatorname{dim}_{\mathbb{C}} H^{1}(X, \mathbb{C})<\infty$, and for each $\delta \in H^{1}(X, \mathbb{C})$ there is a holomorphic 1-form with polynomial coefficients $\omega_{\delta}$ such that $\delta(c)=\int_{c} \omega_{\delta}$ for any $c \in H_{1}(X, \mathbb{C})$.

Corollary 2.15 Let $\Gamma_{n} \subset X$ where $X$ satisfies either condition (1) or (2). Suppose that there is a continuous map $\widetilde{A}_{n}: R \rightarrow X$ of an open neighbourhood $R \subset \mathbb{C}$ of $S^{1}$ such that $\left.\widetilde{A}_{n}\right|_{S^{1}}=A_{n}$, and $\widetilde{A}_{n}^{-1}(x)$ is finite for any $x \in \widetilde{A}_{n}(R)$. Then the monodromy $\widetilde{\rho}_{n}$ is trivial if and only if $A_{n}=A_{1 n} \circ A_{2 n}$ where $A_{2 n}: S^{1} \rightarrow \mathbb{D}$ is a continuous map into the unit disk $\mathbb{D} \subset \mathbb{C}$, locally Lipschitz outside a finite set, and $A_{1 n}: \mathbb{D} \rightarrow \mathbb{C}^{n}$ is a finite holomorphic map.

Remark 2.16 (1) In the proof of Corollary 2.15 we give separate arguments for assumptions (1) and (2). However, it is possible to show that condition (1) can be reduced to the case of condition $(2)$.

(2) We will also show that if $\widetilde{A}_{n}$ is holomorphic then $A_{2 n}$ can be extended to a holomorphic map of an open neighbourhood of $S^{1}$ into $\mathbb{D}$.

(3) Note that the hypotheses of the corollary are valid for $X$ a complex algebraic curve.

(4) In the case when $A_{n}: S^{1} \rightarrow X$ is a Lipschitz embedding and $\Gamma_{n}=A_{n}\left(S^{1}\right)$ lies outside singularities of $X$, one can obtain under the hypotheses of Corollary 2.15 that $A_{n}=A_{1 n} \circ A_{2 n}$ where $A_{2 n}: S^{1} \rightarrow \mathbb{D}$ is a Lipschitz embedding and $A_{1 n}: \mathbb{D} \rightarrow \mathbb{C}^{n}$ is a holomorphic map, one-to-one outside a finite set. 
Corollary 2.17 Suppose that the coefficients $a_{1}, \ldots, a_{n}$ in (2.8) are trigonometric polynomials. Then the monodromy $\widetilde{\rho}_{n}$ is trivial if and only if there is a trigonometric polynomial $q$ and polynomials $p_{1}, \ldots, p_{n} \in \mathbb{C}[z]$ such that

$$
\widetilde{a}_{i}(x)=p_{i}(q(x)), \quad x \in S^{1}, \quad 1 \leq i \leq n .
$$

2.5. Finally, we formulate two finiteness results showing that in certain cases the triviality of some $\widetilde{\rho}_{N}$ implies that of $\widetilde{\rho}$.

Theorem 2.18 Suppose that the coefficients $a_{1}, a_{2}, \ldots$, in equation (2.3) are continuous functions such that each $\widetilde{a}_{i}:=\int_{0}^{x} a_{i}(s) d s$ is $2 \pi$-periodic. Let $A_{k}: S^{1} \rightarrow \mathbb{C}^{k}$, $A_{k}(x):=\left(\widetilde{a}_{1}(x), \ldots, \widetilde{a}_{k}(x)\right)$, and $\Gamma_{k}=A_{k}\left(S^{1}\right)$. Suppose that there is an integer $n$ such that $\Gamma_{n}$ is a piecewise smooth curve, the number of critical values of $A_{n}$ is finite, and $A_{n}^{-1}(x)$ is finite for any $x \in \Gamma_{n}$. If also $\widehat{\Gamma}_{k}=\Gamma_{k}$ for any $k$, then there is an integer $N \geq 1$ such that the triviality of $\widetilde{\rho}_{N}$ implies the triviality of $\widetilde{\rho}$.

Remark 2.19 The hypotheses of the theorem are fulfilled if, e.g., all $a_{i}$ are real continuous functions and some $\widetilde{a}_{n}$ is a non-zero analytic function.

Theorem 2.20 Suppose that there are $2 \pi$-periodic Lipschitz functions $b_{1}, \ldots, b_{k}$ such that each coefficient $a_{i}$ in equation (2.3) is the uniform limit of functions of the form $\sum_{j=1}^{k} p_{j i}\left(b_{1}, \ldots, b_{k}\right) \cdot b_{j}^{\prime}$, where $p_{j i} \in \mathbb{C}\left[z_{1}, \ldots, z_{k}\right]$ are holomorphic polynomials and $b_{j}^{\prime}$ is the derivative of $b_{j}$. Then the triviality of the monodromy of the equation $F^{\prime}(x)=\left(\sum_{i=1}^{k} b_{i}^{\prime}(x) t^{i} X_{1} X_{2}^{i-1}\right) \cdot F(x)$ implies that the monodromy $\widetilde{\rho}$ for (2.3) is trivial.

Corollary 2.21 Let now $H(x, y) \in \mathbb{C}[x, y]$ be a homogeneous polynomial. For any holomorphic functions $P_{1}, P_{2}$ defined in an open neighbourhood of $0 \in \mathbb{C}$ we define $A(x, y):=P_{1}(H(x, y))$, and $B(x, y):=P_{2}(H(x, y))$. Then the vector field

$$
\left\{\begin{array}{l}
\dot{x}=-y-x y \frac{\partial A(x, y)}{\partial x}+x^{2} \frac{\partial A(x, y)}{\partial y}-y B(x, y) \\
\dot{y}=x-y^{2} \frac{\partial A(x, y)}{\partial x}+x y \frac{\partial A(x, y)}{\partial y}+x B(x, y)
\end{array}\right.
$$

determines a center.

The next result deals with symmetries of vector fields. Since the center problem is invariant under rotations, it suffices to consider a particular case of the symmetry. This result first was obtained by Poincaré $[\mathrm{P}]$ by a different argument.

Corollary 2.22 Let $F(x, y), G(x, y)$ be analytic functions defined in an open neighbourhood of $0 \in \mathbb{R}^{2}$ such that their Taylor expansions at 0 do not contain constant and linear terms. Suppose $F(x,-y)=-F(x, y)$ and $G(x,-y)=G(x, y)$. Then the vector field $\dot{x}=-y+F(x, y), \dot{y}=x+G(x, y)$ determines a center. 
Example 2.23 The field (1.2) with homogeneous $F(x, y)$ and $G(x, y)$ of degree 2 can be written in the Dulac-Kapteyn form with 5 parameters instead of 6 by using an appropriate rotation of the plane:

$$
\left\{\begin{array}{l}
\dot{x}=-y-\lambda_{3} x^{2}+\left(2 \lambda_{2}+\lambda_{5}\right) x y+\lambda_{6} y^{2} \\
\dot{y}=x+\lambda_{2} x^{2}+\left(2 \lambda_{3}+\lambda_{4}\right) x y-\lambda_{2} y^{2}
\end{array}\right.
$$

It has been established by H. Dulac that the center set for (2.9) consists of 4 components described as follows (cf. [Si]).

(1) Lotka-Volterra component: $\lambda_{3}=\lambda_{6}$;

(2) Symmetric component: $\lambda_{2}=\lambda_{5}=0$;

(3) Hamiltonian component: $\lambda_{4}=\lambda_{5}=0$;

(4) Darboux component: $\lambda_{5}=\lambda_{4}+5 \lambda_{3}-5 \lambda_{6}=\lambda_{3} \lambda_{6}-2 \lambda_{6}^{2}-\lambda_{2}^{2}=0$.

Next, passing to polar coordinates in (2.9) we obtain

$$
\frac{d r}{d \phi}=\frac{f(\phi) r^{2}}{1+g(\phi) r}
$$

where $f(\phi):=\frac{x F(x, y)+y G(x, y)}{r^{3}}, g(\phi):=\frac{x G(x, y)-y F(x, y)}{r^{3}}, x=r \cos \phi, y=r \sin \phi$. Let us investigate the universal center conditions for equation (2.10). If (2.10) determines a universal center then the first integrals $I(f)$ and $I(f g)$ of $f$ and $f g$ satisfy the relations of Corollary 2.17. This easily implies that $I(f)$ and $g$ satisfy the same relations. Going back to the functions $F$ and $G$ we obtain a planar polynomial vector field of the form of Corollary 2.21. Now $H$ is a linear homogeneous polynomial, and $P_{1}, P_{2}$ are linear polynomials without constant terms. A simple computation shows that in this case for the equation (2.9) we have

(A) $\lambda_{3}-\lambda_{6}=4 \lambda_{2}+\lambda_{5}=4 \lambda_{3}+\lambda_{4}=0$ (a linear two-dimensional subspace in the Lotka-Volterra component);

(B) $\lambda_{2}=\lambda_{5}$ (Symmetric component).

Remark 2.24 We can further simplify equation (2.10) by the application of the Cherkas transformation [Che], $r(\phi)=\frac{\rho(\phi)}{1-g(\phi) \rho(\phi)}$. Then we get the Abel differential equation

$$
\frac{d \rho}{d \phi}=p(\phi) \rho^{2}+q(\phi) \rho^{3}
$$

where $p(\phi)=f(\phi)+g^{\prime}(\phi)$ and $q(\phi)=-f(\phi) g(\phi)$. Moreover, since the Cherkas transformation and its inverse are regular at $r=\rho=0$, the center sets of $(2.10)$ and (2.11) coincide. Let $P(\phi)=\int_{0}^{\phi} p(s) d s$ and $Q(\phi)=\int_{0}^{\phi} q(s) d s$. In case equation (2.11) determines a center, $P$ and $Q$ are trigonometric polynomials. It was shown by Blinov $[\mathrm{Bl}]$ that the Hamiltonian and the Symmetric components after performing the Cherkas transformation determine universal centers for (2.11) and the other two components do not. Here for the Hamiltonian component we have $Q=-\frac{3}{16} P^{2}+\frac{\lambda_{2}}{2} P$, and for the Symmetric component $P$ and $Q$ are polynomials in $\sin (\phi)$. Then the required result follows from Corollary 2.17.

Thus the Hamiltonian component (which is a universal center for (2.11)) can be obtained as a result of an additional non-linear change of variables in (2.10).

The results of Section 2 will be proved in Sections 4-8. 


\section{Algebraic Model for the Center Problem.}

3.1. Now we consider the Center Problem for (1.1) from an algebraic point of view.

Let us introduce a multiplication $*: X \times X \rightarrow X$ as follows.

Given $a=\left(a_{1}, a_{2}, \ldots\right)$ and $b=\left(b_{1}, b_{2}, \ldots\right)$ from $X$ we define

$$
a * b=\left(a_{1} * b_{1}, a_{2} * b_{2}, \ldots\right) \in X \quad \text { and } \quad a^{-1}=\left(a_{1}^{-1}, a_{2}^{-1}, \ldots\right) \in X
$$

where for any $i \in \mathbb{N}$,

$$
\left(a_{i} * b_{i}\right)(t)=\left\{\begin{array}{ccc}
2 a_{i}(2 t) & \text { if } & 0<t \leq \pi \\
2 b_{i}(2 t-2 \pi) & \text { if } & \pi<t \leq 2 \pi
\end{array}\right.
$$

and

$$
a_{i}^{-1}(t)=-a_{i}(2 \pi-t), \quad 0<t \leq 2 \pi .
$$

From Proposition 2.6 it follows that equation (1.1) corresponding to $a \in X$ determines a universal center, if all non-constant basic iterated integrals vanish at $a$, i.e., $I_{i_{1}, \ldots, i_{k}}(a)=0$ for all possible $i_{1}, \ldots, i_{k}, k \in \mathbb{N}$.

We say that $a, b \in X$ are equivalent (written, $a \sim b$ ) if $a * b^{-1} \in \mathcal{U}$.

Proposition 3.1 The relation $\sim$ is reflexive, symmetric and transitive so that $X$ partitions into mutually disjoint equivalence classes.

By $G(X)$ we denote the set of all the equivalence classes, and by $[a]$ the class containing $a \in X$. The element $a$ will be called a representative of the class $[a]$.

Proposition 3.2 The following relations hold:

(1) if $a \sim a^{\prime}$ and $b \sim b^{\prime}$ then $a * b \sim a^{\prime} * b^{\prime}$;

(2) $(a * b) * c \sim a *(b * c)$;

(3) $a * 0 \sim 0 * a$;

(4) $a * a^{-1} \sim 0 \sim a^{-1} * a$.

From (1) it follows that the class $[a * b]$ depends only on classes $[a]$ and $[b]$. Therefore the formula

$$
[a] \cdot[b]=[a * b]
$$

determines a multiplication in $G(X)$. Also, from properties (2), (3) and (4) we obtain that $G(X)$ is a group with respect to this multiplication. The class $[0]=e$ is the unit of the group $G(X)$ and the class $\left[a^{-1}\right]$ is the inverse to the class $[a]$.

Proposition 3.3 If $a, b \in X$ are such that $a \sim b$, then $I(a)=I(b)$ for any iterated integral $I$. 
Thus every such $I$ determines a function $\widehat{I}: G(X) \rightarrow \mathbb{C}, \widehat{I}([a]):=I(a)$, which also will be referred to as an iterated integral on $G(X)$. The algebra of iterated integrals on $G(X)$ will be denoted by $\mathcal{I}(G(X))$.

Next we introduce a topology $\tau$ on $G(X)$ as follows.

Given $g \in G(X)$ the base of open neighbourhoods at $g$ consists of all possible sets of the form

$$
\left\{h \in G(X):\left|\widehat{I}_{1}(h)-\widehat{I}_{1}(g)\right|<\epsilon, \ldots,\left|\widehat{I}_{k}(h)-\widehat{I}_{k}(g)\right|<\epsilon\right\}
$$

where $I_{1}, \ldots, I_{k}$ are basic iterated integrals and $\epsilon$ is a positive number. This is the weakest topology in which all functions $\widehat{I}$ are continuous.

We say that a group $G$ is residually torsion free nilpotent if the set of finitedimensional unipotent representations separates elements of $G$. (For instance, a free group with at most countable number of generators satisfies this property.)

The following result describes certain properties of $G(X)$.

Theorem 3.4 (1) The family $\mathcal{I}(G(X))$ separates points on $G(X)$.

(2) $(G(X), \cdot, \tau)$ is a separable topological group.

(3) $G(X)$ is metrizable and is the union of an increasing sequence of compact subsets.

(4) $G(X)$ is contractible to a point, arcwise connected, locally simply and arcwise connected.

(5) $G(X)$ is residually torsion free nilpotent.

3.2. Let $G_{c}[[r]]$ be the set of complex power series of the form $f(r)=r+\sum_{k=1}^{\infty} d_{k} r^{k+1}$ each convergent in some open neighbourhood of $0 \in \mathbb{C}$. It is easy to see that $G_{c}[[r]]$ is a group with the multiplication defined by the composition of series. Consider the functions $d_{k}: G_{c}[[r]] \rightarrow \mathbb{C}$ such that $d_{k}(f)$ equals the $k+1$-st coefficient of the Taylor expansion of $f$ at 0 . Let $\tau^{\prime}$ be the weakest topology on $G_{c}[[r]]$ in which all functions $d_{k}$ are continuous. Then we prove

Proposition $3.5\left(G_{c}[[r]], \circ, \tau^{\prime}\right)$ is a separable topological group. The family of functions $d_{k}$ separates points on $G_{c}[[r]]$. Moreover, $G_{c}[[r]]$ is contractible to a point, arcwise connected, locally simply and arcwise connected, and residually torsion free nilpotent.

Next we will confine our attention to the first return map $P(a)$ of equation (1.1) corresponding to $a \in X$. It is clear that $P(a) \in G_{c}[[r]]$. The following property of the map $P$ is the core of our construction.

Theorem 3.6 The identity

$$
P(a * b)=P(b) \circ P(a)
$$

holds for any $a, b \in X$. 
From here and the fact that $P(a)(r) \equiv r$ for any $a \in \mathcal{U}$ we obtain that there exists a map $\widehat{P}: G(X) \rightarrow G_{c}[[r]]$ such that $\widehat{P}([a]):=P(a)$. Then we will prove

Theorem 3.7 The map $\widehat{P}: G(X) \rightarrow G_{c}[[r]]$ is a surjective homomorphism of topological groups. The kernel $\widehat{\mathcal{C}} \subset G(X)$ of $\widehat{P}$ corresponds to the image of the center set $\mathcal{C} \subset X$ in $G(X)$. Moreover, $\widehat{\mathcal{C}}$ is a non-trivial, normal, closed, contractible to a point, arcwise connected, locally simply and arcwise connected subgroup of $G(X)$.

Let us consider the quotient group $Q(X):=G(X) / \widehat{\mathcal{C}}$. By $\pi: G(X) \rightarrow Q(X)$ we denote the quotient homomorphism. According to Proposition 3.3, the Taylor coefficients $c_{n}$ of the first return map $P$ (see Theorem 2.2) satisfy $c_{n}(a)=c_{n}(b)$ for $a \sim b$. Therefore they can be viewed as functions on $G(X)$ (written, $\widehat{c}_{n}$ ).

Proposition 3.8 Every function $\widehat{c}_{n}$ is constant on fibres of the map $\pi$ and therefore determines a function $\bar{c}_{n}: Q(X) \rightarrow \mathbb{C}$ such that $\bar{c}_{n} \circ \pi=\widehat{c}_{n}$.

Let $\tau^{\prime \prime}$ be the weakest topology on $Q(X)$ in which all functions $\bar{c}_{n}$ are continuous.

Theorem 3.9 $Q(X)$ equipped with $\tau^{\prime \prime}$ is a topological group. Moreover, the homomorphism $\widehat{P}$ determines an isomorphism $\bar{P}: Q(X) \rightarrow G_{c}[[r]]$ defined by $\widehat{P}=\bar{P} \circ \pi$.

Now, given $f(r)=r+\sum_{k=1}^{\infty} d_{k} r^{k+1} \in G_{c}[[r]]$ we define $a(f)=\left(a_{1}, a_{2}, \ldots\right) \in X$ from the identity of formal power series

$$
\sum_{i=1}^{\infty} a_{i}(x) t^{i+1}=\frac{\sum_{k=1}^{\infty}\left(d_{k} / 2 \pi\right) t^{k+1}}{1+\sum_{k=1}^{\infty}(k+1) d_{k}(1-x / 2 \pi) t^{k}} \quad \text { for } \quad x \in(0,2 \pi]
$$

and further extended by periodicity. Let $[a(f)] \in G(X)$ be the image of $a(f)$. By $T$ we denote the map $f \mapsto[a(f)]$. Then we have

Theorem 3.10 The map $T: G_{c}[[r]] \rightarrow G(X)$ is a continuous embedding such that $\widehat{P} \circ T=i d$. Moreover, the map $\widetilde{T}: G_{c}[[r]] \times \widehat{\mathcal{C}} \rightarrow G(X)$ defined by $\widetilde{T}(f, g):=T(f) \cdot g$ is a homeomorphism.

3.3. In this part we study some naturally defined subgroups of $G(X)$.

Let us consider the subspace $X_{s} \subset X$ consisting of $a=\left(a_{1}, a_{2}, \ldots\right) \in X$ such that every $a_{i}$ is a piecewise continuous complex-valued function on $S^{1}$. Clearly, for any $a, b \in X_{s}$ we have $a * b \in X_{s}$ and $a^{-1} \in X_{s}$. Let $G_{s}(X)$ be the image of $X_{s}$ in $G(X)$. Then $G_{s}(X)$ is a subgroup of $G(X)$. Similarly, let $X_{a} \subset X$ be the subspace of elements $a=\left(a_{1}, a_{2}, \ldots\right) \in X$ such that every $a_{i}$ is a piecewise analytic complexvalued function on $S^{1}$. Then the image $G_{a}(X)$ in $G(X)$ is a subgroup, as well. Also, $G_{a}(X) \subset G_{s}(X)$. In what follows we consider these groups with relative topologies induced by $\tau$. We will prove

Proposition $3.11 G_{s}(X)$ and $G_{a}(X)$ satisfy properties similar to (1),(2),(4),(5) of Theorem 3.4.

Let $\widehat{\mathcal{C}}_{s}:=\widehat{\mathcal{C}} \cap G_{s}(X)$ and $\widehat{\mathcal{C}}_{a}:=\widehat{\mathcal{C}} \cap G_{a}(X)$. Then $\widehat{\mathcal{C}}_{s}$ and $\widehat{\mathcal{C}}_{a}$ are closed normal subgroups of the corresponding groups. 
Theorem 3.12 (1) $\left.\widehat{P}\right|_{G_{s}(X)}: G_{s}(X) \rightarrow G_{c}[[r]]$ and $\left.\widehat{P}\right|_{G_{a}(X)}: G_{a}(X) \rightarrow G_{c}[[r]]$ are surjective homomorphisms of the topological groups whose kernels are $\widehat{\mathcal{C}}_{s}$ and $\widehat{\mathcal{C}}_{a}$, respectively.

(2) The quotient groups $G_{s}(X) / \widehat{\mathcal{C}}_{s}$ and $G_{a}(X) / \widehat{\mathcal{C}}_{a}$ are isomorphic to $Q(X)$.

(3) The range of the map $T$ from Theorem 3.10 is a subset of $G_{a}(X)$, thus, maps $\widetilde{T}_{s}: G_{c}[[r]] \times \widehat{\mathcal{C}}_{s} \rightarrow G_{s}(X), \widetilde{T}_{s}(f, g):=T(f) \cdot g$, and $\widetilde{T}_{a}: G_{c}[[r]] \times \widehat{\mathcal{C}}_{a} \rightarrow G_{a}(X)$, $\widetilde{T}_{a}(f, g):=T(f) \cdot g$, are homeomorphisms.

We say that a subset $S \subset G(X)$ is defined over a field $\mathbb{F}, \mathbb{Q} \subseteq \mathbb{F} \subseteq \mathbb{C}$, if $\widehat{I}(s) \in \mathbb{F}$ for every $s \in S$ and any basic iterated integral $\widehat{I}$ on $G(X)$.

Let $X_{\mathbb{R}} \subset X$ be the subspace of elements $a=\left(a_{1}, a_{2}, \ldots\right) \in X$ such that every $a_{i}$ is a real-valued function. Then the corresponding subgroup $G_{\mathbb{R}}(X) \subset G(X)$ is defined over $\mathbb{R}$. Also, we consider subspaces $X_{s, \mathbb{R}}:=X_{\mathbb{R}} \cap X_{s}$ and $X_{a, \mathbb{R}}:=$ $X_{\mathbb{R}} \cap X_{a}$ of real-valued piecewise continuous and piecewise analytic sequences of functions, respectively. By $G_{s, \mathbb{R}}(X)$ and $G_{a, \mathbb{R}}(X)$ we denote the subgroups of $G_{\mathbb{R}}(X)$ corresponding to $X_{s, \mathbb{R}}$ and $X_{a, \mathbb{R}}$. By definition, they are defined over $\mathbb{R}$. In what follows we consider these groups with topologies induced by $\tau$. Let $G_{c, \mathbb{R}}[[r]] \subset G_{c}[[r]]$ be the subgroup of convergent power series with real Taylor coefficients. We consider $G_{c, \mathbb{R}}[[r]]$ with the topology induced by $\tau^{\prime}$. Also, we set $\widehat{\mathcal{C}}_{\mathbb{R}}:=\widehat{\mathcal{C}} \cap G_{\mathbb{R}}(X), \widehat{\mathcal{C}}_{s, \mathbb{R}}:=$ $\widehat{\mathcal{C}}_{s} \cap G_{\mathbb{R}}(X), \widehat{\mathcal{C}}_{a, \mathbb{R}}:=\widehat{\mathcal{C}}_{a} \cap G_{\mathbb{R}}(X), Q_{\mathbb{R}}(X):=G_{\mathbb{R}}(X) / \widehat{\mathcal{C}}_{\mathbb{R}}$. All the above groups defined over $\mathbb{R}$ will be called the real analogs of the corresponding groups defined over $\mathbb{C}$.

Theorem 3.13 The results similar to those of Proposition 3.11 and Theorem 3.12 are valid for the real analogs of the corresponding groups.

Let us consider another construction of subgroups of $G(X)$ defined over various number fields that reveals certain arithmetic properties of the center set.

Given a sequence $b:=\left\{b_{k}\right\}_{k \geq 1} \subset L^{\infty}\left(S^{1}\right)$ such that $\sup _{k, x \in S^{1}}\left|b_{k}(x)\right| \leq 1$, let $\hat{b}=\left(\hat{b}_{1}, \hat{b}_{2}, \ldots\right) \in X$ be the set of all possible monomials $b_{i_{1}}^{\alpha_{1}} \ldots b_{i_{n}}^{\alpha_{n}}, \alpha_{1}, \ldots, \alpha_{n} \in \mathbb{Z}_{+}$, $i_{1}, \ldots, i_{n}, n \in \mathbb{N}$, arranged in a sequence. Suppose $[\hat{b}] \in G(X)$ is defined over a field $\mathbb{F}$. Given a number field $\mathbb{F}^{\prime} \supseteq \mathbb{F}$, let $\mathcal{A}_{\mathbb{F}^{\prime}}(b)$ be the algebra over $\mathbb{F}^{\prime}$ generated by $b$, that is $f \in \mathcal{A}_{\mathbb{F}^{\prime}}(b)$ if there exist $\hat{b}_{i_{1}}, \ldots, \hat{b}_{i_{k}}$ such that $f=l\left(\hat{b}_{i_{1}}, \ldots, \hat{b}_{i_{k}}\right)$, where $l$ is a linear function in $\hat{b}_{i_{1}}, \ldots, \hat{b}_{i_{k}}$ with coefficients from $\mathbb{F}^{\prime}$. Let $X_{\mathbb{F}^{\prime}}(b) \subset X$ be the subset of sequences $a=\left(a_{1}, a_{2}, \ldots\right)$ such that every $a_{i} \in \mathcal{A}_{\mathbb{F}^{\prime}}(b)$. By $G_{\mathbb{F}^{\prime}}(b) \subset G(X)$ we denote the subgroup generated by images of all possible elements from $X_{\mathbb{F}^{\prime}}(b)$.

Proposition 3.14 The subgroup $G_{\mathbb{F}^{\prime}}(b)$ is defined over the field $\mathbb{F}^{\prime}$.

Let $\widehat{\mathcal{C}}_{\mathbb{F}^{\prime}}(b):=\widehat{\mathcal{C}} \cap G_{\mathbb{F}^{\prime}}(b)$. Suppose $\sigma: \mathbb{F}^{\prime} \rightarrow \mathbb{F}^{\prime}$ is a field automorphism over $\mathbb{F}$.

Proposition 3.15 The automorphism $\sigma$ generates a group automorphism $\widetilde{\sigma}: G_{\mathbb{F}^{\prime}}(b) \rightarrow G_{\mathbb{F}^{\prime}}(b)$ continuous in the discrete topology of $G_{\mathbb{F}^{\prime}}(b)$ such that $\widetilde{\sigma}\left(\widehat{\mathcal{C}}_{\mathbb{F}^{\prime}}(b)\right)=\widehat{\mathcal{C}}_{\mathbb{F}^{\prime}}(b)$. 
We say that $P:[0,1] \rightarrow X$ is an $X_{\mathbb{F}^{\prime}}(b)$-polynomial map if $P(t) \in X$ for any $t \in[0,1]$, and $P(t)=c_{1}^{\epsilon_{1}}(t) * \cdots * c_{k}^{\epsilon_{k}}(t)$ where every $c_{i}(t)=\left(c_{1 i}(t), c_{2 i}(t), \ldots\right)$ with $c_{k i}(t)$ polynomials in $t$ whose coefficients are elements from $\mathcal{A}_{\mathbb{F}^{\prime}}(b)$ and every $\epsilon_{l}, 1 \leq l \leq k$, equals either -1 or 1 . The induced map $[P]:[0,1] \rightarrow G(X)$, $[P](t):=[P(t)]$, will be called an $X_{\mathbb{F}^{\prime}}(b)$-polynomial arc.

Clearly, $[P](t) \in G_{\mathbb{F}^{\prime}}(b)$ for any $t \in \mathbb{F}^{\prime} \cap[0,1]$. In the sequel we will show that $[P]:[0,1] \rightarrow G(X)$ is a continuous map (for $G_{\mathbb{F}^{\prime}}(b)$ considered in the relative topology induced by $\tau$ ).

Theorem 3.16 Suppose $[P](0) \notin \widehat{\mathcal{C}}$ and $[P](\xi) \in \widehat{\mathcal{C}}$ for some $\xi \in(0,1]$. Then $\xi$ is algebraic over $\mathbb{F}^{\prime}$.

Example 3.17 (1) Suppose $b=\left\{b_{k}\right\}_{k \geq 1} \subset L^{\infty}\left(S^{1}\right)$ where $b_{2 k-1}(x)=\sin k x$ and $b_{2 k}(x)=\cos k x, k=1,2, \ldots$ Then $[\hat{b}] \in G(X)$ is defined over the field $\mathbb{Q}(\pi)$.

(2) Suppose $b=\left\{b_{k}\right\}_{k \geq 1} \subset L^{\infty}\left(S^{1}\right)$ where every $b_{k}(x):=\chi_{[0,1]}(x) \cdot x^{k-1}$ for $x \in(0,2 \pi]$ and then extended by periodicity. Here $\chi_{[0,1]}$ is the characteristic function of $[0,1]$. Then $[\hat{b}] \in G(X)$ is defined over $\mathbb{Q}$.

Remark 3.18 (1) Let $X_{n}$ be the set of elements $a=\left(a_{1}, \ldots, a_{n}, 0,0, \ldots\right) \in X$. The image of $X_{n}$ in $G(X)$ forms a subgroup $G\left(X_{n}\right)$ with properties similar to those of Theorem 3.4. The projection $p_{n}: X \rightarrow X_{n}, p_{n}\left(\left(a_{1}, a_{2}, \ldots\right)\right):=\left(a_{1}, \ldots, a_{n}, 0,0, \ldots\right)$, induces a surjective homomorphism $p_{n *}: G(X) \rightarrow G\left(X_{n}\right)$. In particular, $G\left(X_{n}\right)$ is isomorphic (in the category of topological groups) to the semidirect product of $G\left(X_{n}\right)$ and the normal subgroup $\operatorname{Ker}\left(p_{n *}\right) \subset G(X)$. Set $\widehat{\mathcal{C}}_{n}:=\widehat{\mathcal{C}} \cap G\left(X_{n}\right)$. From the results of Blinov [Bl] (see Remark 2.24) it follows, e.g., that $\widehat{\mathcal{C}}_{n}$ is a non-trivial subgroup of $G\left(X_{n}\right)$. However, it is a very delicate and difficult problem to describe explicitly the elements of $\widehat{\mathcal{C}}_{n}$.

(2) As before, let $X_{i}$ be the space of all coefficients $a_{i}$ from (1.1). We set $X_{u}:=$ $\prod_{i \geq 1} X_{i}$. Then clearly, $X \subset X_{u}$. The vector space $X_{u}$ parameterizes all equations of type (1.1) with right-hand sides being formal power series in $v$. Also, one can determine a multiplication $*$ on $X_{u}$ similarly to that of on $X$. Considering an equivalence relation on $X_{u}$ similar to that of on $X$, as a quotient set we get a topological group $G\left(X_{u}\right)$ such that $G(X) \subset G\left(X_{u}\right)$. One can show that $G\left(X_{u}\right)$ shares most of the properties similar to those of the group $G(X)$ (see Sections 3.1 and 3.2). In particular, the center set $\widehat{\mathcal{C}}_{u} \subset G\left(X_{u}\right)$ is the kernel of the natural homomorphism $\widehat{P}_{u}: G\left(X_{u}\right) \rightarrow G[[r]]$ corresponding to formal solutions of (1.1). Here $G[[r]]$ is the group of formal complex power series of the form $x+\sum_{i=1}^{\infty} d_{i} r^{i+1}$. Sometimes to get more information about equation (1.1) it is easier to work with $G\left(X_{u}\right)$ instead of $G(X)$, in particular, because we don't require any special estimates there.

The results of Section 3 will be proved in Sections 9-11.

\section{Proofs of Results of Section 2.1.}

In this section we prove Theorems 2.1, 2.2, 2.4 and Corollary 2.3.

4.1. We use the following definitions (see e.g. [Ru, Ch. 7] for possible references). 
Definition 4.1 Let $I \subset \mathbb{R}$ be a closed interval. A map $f: I \rightarrow \mathbb{R}^{n}$ is called Lipschitz if $\operatorname{dist}(f(x), f(y)) \leq C|x-y|$ for any $x, y \in I$. Here dist $(\cdot, \cdot)$ denotes the Euclidean distance on $\mathbb{R}^{n}$. A map $f: J \rightarrow \mathbb{R}^{n}$ defined on an open interval $J \subset \mathbb{R}$ is called locally Lipschitz if it is Lipschitz on each closed subinterval of J.

Let mes $(V)$ denote the Lebesgue measure of $V \subset \mathbb{R}$. We say that $f: I \rightarrow \mathbb{R}$ is absolutely continuous if mes $(f(E))=0$ provided that mes $(E)=0$.

Any Lipschitz $f: I \rightarrow \mathbb{R}$ is absolutely continuous. Also, such $f$ is differentiable almost everywhere (Lebesgue) and its derivative belongs to $L^{\infty}(I)$. The first integral of a function from $L^{\infty}(I)$ is a Lipschitz function. Now, if $f$ is absolutely continuous and $f^{\prime}$ is zero almost everywhere, then $f$ is constant. Therefore derivative of antiderivative of any function from $L^{\infty}(I)$ almost everywhere coincides with this function. Moreover, any Lipschitz $f$ is an antiderivative of $f^{\prime}$. Therefore the Newton-Leibnitz formula $\int_{a}^{b} f^{\prime}(t) d t=f(b)-f(a)$ holds. Similar results are valid for complex-valued Lipschitz functions. These facts are basic when we solve systems of linear differential equations with $L^{\infty}$-coefficients by Picard iteration.

4.2. Let us consider equation (1.1). To make sure that it has a Lipschitz solution defined on $[0,2 \pi]$ we assume that there is a constant $l<\infty$, such that for any $i$

$$
\sup _{x \in[0,2 \pi]}\left|a_{i}(x)\right| \leq l^{i}
$$

First observe that the substitution $r(x)=t \cdot v(x)$ transforms (1.1) to

$$
v^{\prime}=\sum_{i=1}^{\infty} a_{i}(x) t^{i} v^{i+1}
$$

and that equation (1.1) determines a center if and only if (4.2) determines a center for any sufficiently small $t$. Multiplying (4.2) by $v^{k-1}$ we obtain

$$
\left(v^{k}\right)^{\prime}=\sum_{i=1}^{\infty}\left(k a_{i}(x) t^{i}\right) v^{i+k}
$$

Let $V$ be the linear space spanned by vectors $e_{i}=(0, \ldots, 0,1,0, \ldots)$ with 1 at the $i^{\text {th }}$ place. We set $y_{i}=v^{i} e_{i}$ and $Y=\left(y_{1}, y_{2}, \ldots\right)$. Combining equations (4.3) for all $k$ we obtain a formal linear system

$$
Y^{\prime}=\left(\sum_{i=1}^{\infty} A_{i} a_{i}(x) t^{i}\right) Y
$$

where $A_{i}: V \rightarrow V$ are linear operators. Let $\mathbb{C}[[z]]$ be the algebra of formal power series with complex coefficients

$$
f(z)=\sum_{k=0}^{\infty} c_{k} z^{k}
$$

We identify $V$ with $\mathbb{C}[[z]]$ so that $e_{n}$ coincides with $z^{n-1}$. Let $D: \mathbb{C}[[z]] \rightarrow \mathbb{C}[[z]]$ be the differentiation operator

$$
(D f)(z):=\sum_{k=0}^{\infty}(k+1) c_{k+1} z^{k}
$$


and $L: \mathbb{C}[[z]] \rightarrow \mathbb{C}[[z]]$ be the left translation operator

$$
(L f)(z):=\sum_{k=0}^{\infty} c_{k+1} z^{k} .
$$

Then we have

\section{Proposition 4.2}

$$
A_{i}=D L^{i-1} .
$$

Thus (4.4) can be written as

$$
Y^{\prime}=\left(\sum_{i=1}^{\infty} a_{i}(x) t^{i} D L^{i-1}\right) Y .
$$

Proof. Applying $D L^{i-1}$ to the function $z^{k}$ we have 0 if $k \leq i-1$ and otherwise

$$
D L^{i-1}\left(z^{k}\right)=D\left(z^{k-i+1}\right)=(k-i+1) z^{k-i} .
$$

Now, by definition, $A_{i}=\left(a_{k, l}^{i}\right)$ is an infinite matrix such that $a_{s, i+s}^{i}=s$ for any $s \in \mathbb{Z}_{+}$and $a_{k, l}^{i}=0$ otherwise. Thus we have

$$
A_{i}\left(e_{k+1}\right)=\sum a_{j, k+1}^{i} e_{j}=(k-i+1) e_{k-i+1} .
$$

Further, by $\mathcal{A}(D, L)[[t]]$ we denote the associative algebra of formal power series

$$
R(t)=\sum_{k=0}^{\infty} p_{k}(D, L, I) t^{k}
$$

where $p_{i}(D, L, I)$ are polynomials with complex coefficients in variables $D, L$ and $I$, where $I: \mathbb{C}[[z]] \rightarrow \mathbb{C}[[z]]$ is the identity operator. Let $G(D, L)[[t]]$ be the group of invertible elements of $\mathcal{A}(D, L)[[t]]$, and $\rho^{\prime}: \mathbb{Z} \rightarrow G(D, L)[[t]]$ be the monodromy of (4.5). We prove that

Lemma 4.3 There is an isomorphism $\Phi: \mathcal{A}(D, L)[[t]] \rightarrow \mathcal{A}\left(S_{1}, S_{2}\right)[[t]]$ uniquely defined by $\Phi(D)=S_{1}$ and $\Phi(L)=S_{2}$. In particular, for the monodromy $\rho$ from Theorem 2.1 we have $\rho=\Phi \circ \rho^{\prime}$.

Proof. Let $\mathcal{A}(D, L)$ be the associative algebra of polynomials with complex coefficients in variables $D, L$ and $I$. By $\mathcal{A}_{0}(D, L)$ we denote the subalgebra of $\mathcal{A}(D, L)$ generated by $D$ and $L$ only. Let $\mathbb{C}(I) \subset \mathcal{A}(D, L)$ be the one-dimensional central subalgebra generated by $I$. Then $\mathcal{A}(D, L)=\mathcal{A}_{0}(D, L) \oplus \mathbb{C}(I)$. First, we prove the following basic relation for $D$ and $L$.

\section{Proposition 4.4}

$$
[D, L]:=D L-L D=-L^{2} .
$$


Proof. It suffices to check the identity for elements $z^{k}$ with $k \geq 2$. Then we have

$$
D L\left(z^{k}\right)=D\left(z^{k-1}\right)=(k-1) z^{k-2}, \quad L D\left(z^{k}\right)=L\left(k z^{k-1}\right)=k z^{k-2} .
$$

Therefore

$$
[D, L]\left(z^{k}\right)=(k-1) z^{k-2}-k z^{k-2}=-z^{k-2}=-L^{2}\left(z^{k}\right) .
$$

Let $\mathcal{A}\left(X_{1}, X_{2}\right)$ be the algebra of polynomials with complex coefficients in free non-commutative variables $X_{1}, X_{2}$. Then there exists a canonical homomorphism $\phi: \mathcal{A}\left(X_{1}, X_{2}\right) \rightarrow \mathcal{A}_{0}(D, L)$ uniquely defined by the relations $\phi\left(X_{1}\right)=D, \phi\left(X_{2}\right)=L$. From Proposition 4.4 it follows that $\left[X_{1}, X_{2}\right]+X_{2}^{2} \in \operatorname{Ker}(\phi)$. Let $J \subset \mathcal{A}\left(X_{1}, X_{2}\right)$ be the two-sided ideal generated by $\left[X_{1}, X_{2}\right]+X_{2}^{2}$. Let $\mathcal{A}\left(S_{1}, S_{2}\right):=\mathcal{A}\left(X_{1}, X_{2}\right) / J$ be the quotient algebra and $\gamma: \mathcal{A}\left(X_{1}, X_{2}\right) \rightarrow \mathcal{A}\left(S_{1}, S_{2}\right)$ be the quotient homomorphism uniquely defined by $\gamma\left(X_{1}\right)=S_{1}$ and $\gamma\left(X_{2}\right)=S_{2}$. Clearly, there is a homomorphism $\widetilde{\phi}: \mathcal{A}\left(S_{1}, S_{2}\right) \rightarrow \mathcal{A}_{0}(D, L)$ such that $\phi=\widetilde{\phi} \circ \gamma$.

Proposition $4.5 \tilde{\phi}: \mathcal{A}\left(S_{1}, S_{2}\right) \rightarrow \mathcal{A}_{0}(D, L)$ is an isomorphism.

Proof. Obviously, any polynomial in $S_{1}$ and $S_{2}$ can be transformed with the help of the basic relation to a polynomial in the canonical form: $P\left(S_{1}, S_{2}\right)=$ $\sum_{1 \leq i, j \leq n} a_{i j} S_{1}^{i} S_{2}^{j}, a_{i j} \in \mathbb{C}$. Suppose, to the contrary, that $\operatorname{Ker}(\widetilde{\phi}) \neq 0$. This means that there is a $P\left(S_{1}, S_{2}\right)$ whose coefficients in the canonical form not all are zeros such that $P(D, L)=0$. Let us write $P\left(S_{1}, S_{2}\right)=\sum_{k=1}^{n} F_{k}\left(S_{1}, S_{2}\right)$, where $F_{k}\left(S_{1}, S_{2}\right)=\sum_{i=0}^{k} a_{i k-i} S_{1}^{i} S_{2}^{k-i}$ is the homogeneous component of degree $k$. Using the identity

$$
D^{i} L^{j}\left(z^{s}\right)=D^{i}\left(z^{s-j}\right)=(s-j)(s-j-1) \cdots(s-j-i+1) z^{s-i-j}
$$

we obtain for $s \geq n$

$$
0=P(D, L)\left(z^{s}\right)=\sum_{k=1}^{n}\left[a_{0 k}+\sum_{j=0}^{k-1} a_{k-j j}(s-j) \cdots(s-k+1)\right] z^{s-k} .
$$

In particular, for any $1 \leq k \leq n$ and any sufficiently big positive integers $s$ we have

$$
a_{0 k}+\sum_{j=0}^{k-1} a_{k-j j}(s-j) \cdots(s-k+1)=0 .
$$

But then the same identity is valid for any $s \in \mathbb{R}$. Substituting in this identity $s=k-1$ we get $a_{0 k}=0$. Then dividing this identity by $(s-k+1)$ and substituting $s=k-2$ we obtain $a_{1 k-1}=0$. Proceeding similarly we finally get $a_{i j}=0$ for all $i, j$. This means that $P\left(S_{1}, S_{2}\right)=0$, the contradiction which proves the proposition.

Now, in order to finish the proof of the lemma it remains to determine

$$
\Phi\left(\sum_{k=0}^{\infty} p_{k}(D, L, I) t^{k}\right)=\sum_{k=0}^{\infty} p_{k}\left(S_{1}, S_{2}, I\right) t^{k}, \quad \sum_{k=0}^{\infty} p_{k}(D, L, I) t^{k} \in \mathcal{A}(D, L)[[t]] .
$$


Then Proposition 4.5 implies that $\Phi$ is an algebraic isomorphism. The factorization $\rho=\Phi \circ \rho^{\prime}$ then easily follows from Picard's iteration formula for solutions of (4.5) and $(2.2)$.

The proof of Lemma 4.3 is complete.

From this lemma it follows that it suffices to prove Theorem 2.1 for equation (4.5) and its monodromy $\rho^{\prime}$.

4.3. Let $\omega(x):=\sum_{i=1}^{\infty} a_{i}(x) t^{i} D L^{i-1}$. The fundamental solution $Y$ of (4.5) can be obtained by the Picard method of successive approximations

$$
Y(x)=I+\sum_{n=1}^{\infty} \int \cdots \int_{0 \leq s_{1} \leq \cdots \leq s_{n} \leq x} \omega\left(s_{n}\right) \cdots \omega\left(s_{1}\right) d s_{n} \cdots d s_{1} .
$$

Let us define the functions

$$
I_{i_{1}, \ldots, i_{k}}(x):=\int \cdots \int_{0 \leq s_{1} \leq \cdots \leq s_{k} \leq x} a_{i_{k}}\left(s_{k}\right) \cdots a_{i_{1}}\left(s_{1}\right) d s_{k} \cdots d s_{1} .
$$

Then it is easy to see that

$$
Y(x)=\sum_{i=0}^{\infty} p_{i}(x ; D, L) t^{i}
$$

where $p_{0}=I$ and $p_{i}(x ; D, L)=\sum_{i_{1}+\ldots+i_{k}=i} I_{i_{1}, \ldots, i_{k}}(x)\left(D L^{i_{k}-1}\right) \cdots\left(D L^{i_{1}-1}\right)$ is a homogeneous polynomial of degree $i$ in $D, L$ whose coefficients are locally Lipschitz functions in $x \in \mathbb{R}$.

Now, the solution of (4.5) with initial value $Y(0)=\sum_{i=0}^{\infty} y_{i} z^{i} \in \mathbb{C}[[z]]$ is defined by

$$
Y(x ; z, t, Y(0)):=Y(x) \cdot Y(0) .
$$

From (4.8) it follows that

$$
Y(x ; z, t, Y(0))=\sum_{i=0}^{\infty} y_{i}(x ; z) t^{i}
$$

where each $y_{i}(x ; z)$ is a formal power series in $z$ whose coefficients are locally Lipschitz functions in $x \in \mathbb{R}$. Also, it follows from the results of Section 2.1 that the solution of (4.5) of this form with the initial value $Y(0)$ is unique.

Further we study the question when $Y(x ; z, t, Y(0))$ is a continuous function in $x, z$ and $t$ for $|z|,|t|$ small enough.

Theorem 4.6 Let $Y(0)=\sum_{i=0}^{\infty} y_{i} z^{i}$ be such that $\left|y_{i}\right|<r^{i+1}$ and $r<\frac{e^{-2 \pi}}{2 \tilde{l}}$ where $\tilde{l}:=\max \{1, l\}$. Then the solution $Y(x ; z, t, Y(0))$ of $(4.5)$ is a function continuous in $x \in[0,2 \pi]$ and holomorphic in $(t, z) \in \mathbb{D}_{1 / 2} \times \mathbb{D}_{1 / 2}$. Here $\mathbb{D}_{R}:=\{z \in \mathbb{C}:|z|<R\}$.

Proof. First, let us introduce some notation. Let $\mathbb{R}[[z]]$ be the algebra of formal power series with real coefficients, and $C_{+} \subset \mathbb{R}[[z]$ be the cone of series with nonnegative coefficients. For $y_{1}, y_{2} \in \mathbb{R}[[z]]$ we write $y_{1} \leq y_{2}$ if $y_{2}-y_{1} \in C_{+}$. Further, let $\mathcal{A}_{\mathbb{R}}(D, L)[[t]] \subset \mathcal{A}(D, L)[[t]]$ be the subalgebra of elements $R(t)=\sum_{k=0}^{\infty} p_{k}(D, L, I) t^{k}$ with $p_{k}(x, y, z)$ real polynomials. Let $R_{i}(t)=\sum_{k=0}^{\infty} p_{i k}(D, L, I) t^{k} \in \mathcal{A}_{\mathbb{R}}(D, L)[[t]]$, 
$i=1,2$. We write $R_{1} \leq R_{2}$ if $p_{1 i}(D, L, I)(f) \leq p_{2 i}(D, L, I)(f)$ for any $f \in$ $C_{+}$. Finally, for any $R(t)=\sum_{k=0}^{\infty} p_{k}(D, L, I) t^{k} \in \mathcal{A}(D, L)[[t]]$ we define $|R|(t)=$ $\sum_{k=0}^{\infty} \tilde{p}_{k}(D, L, I) t^{k} \in A_{\mathbb{R}}(D, L)[[t]]$ such that each coefficient of $\tilde{p}_{k}$ is the modulus of the corresponding coefficient of $p_{k}$. Below we use also that $D, L$ and $I$ map $C_{+}$to itself.

Let $\omega(x)$ be the form in (4.5). Then

$$
|\omega(x)|=\sum_{i=1}^{\infty}\left|a_{i}(x)\right| D L^{i-1} t^{i} .
$$

Further, according to (4.1),

$$
|\omega(x)| \leq \widetilde{\omega}:=\sum_{i=1}^{\infty} l^{i} \cdot D L^{i-1} t^{i}
$$

Let us consider the equation

$$
Y^{\prime}=\widetilde{\omega} \cdot Y
$$

By $\tilde{Y}(x ; z, t)=\sum_{i=0}^{\infty} \widetilde{y}_{i}(x ; z) t^{i}$ we denote the solution of $(4.9)$ with the initial value $\tilde{Y}(0)=\sum_{i=0}^{\infty} r^{i+1} z^{i}$. Let $Y(x ; z, t, Y(0))=\sum_{i=0}^{\infty} y_{i}(x ; z) t^{i}$ be the solution of $(4.5)$ with the initial value $Y(0)$ satisfying the hypothesis of the theorem. Suppose $y_{i}(x ; z)=\sum_{k=0}^{\infty} w_{k i}(x) z^{k}$ and $\widetilde{y}_{i}(x ; z)=\sum_{k=0}^{\infty} \widetilde{w}_{k i}(x) z^{k}$. Then from the estimate $|\omega(x)| \leq \widetilde{\omega}$ and from the formula for the fundamental solution (4.8) we obtain that

$$
\left|w_{k i}(x)\right| \leq \widetilde{w}_{k i}(x)
$$

Thus in order to prove the theorem it suffices to produce good estimates of each $\widetilde{w}_{k i}$.

Notice that (4.9) can be obtained from the equation

$$
v^{\prime}=\sum_{i=1}^{\infty} l^{i} t^{i} v^{i+1}
$$

similarly as we derived equation (4.5) from (4.2). Then if $v(x ; t)$ is the solution of (4.10) with initial value $v(0 ; t)=r$, the solution of (4.9) with the initial value $\tilde{Y}(0)=\sum_{i=0}^{\infty} r^{i+1} z^{i}$ is $\tilde{Y}(x ; z, t)=\sum_{i=0}^{\infty} v(x ; t)^{i+1} z^{i}$. Now from the Gronwall-type inequality we obtain that

$$
|v(x ; t)| \leq r e^{x}, \quad x \in[0,2 \pi],
$$

provided that $|t| \leq 1$ and $|v(x ; t)| \leq \frac{1}{2 \hat{l}}$. In particular, this is true if $r \leq \frac{e^{-2 \pi}}{2 \hat{l}}$. Also, for this estimate of $r$ from Picard iteration we get that $v(x, t)$ is a holomorphic function in $t \in \mathbb{D}_{1}$ for any fixed $x \in[0,2 \pi]$. Let $v(x ; t)^{i+1}=\sum_{k=0}^{\infty} c_{k i}(x) t^{k}$. Then from the Cauchy estimates for derivatives of $v(x ; t)$ we have $\left|c_{k i}(x)\right| \leq \frac{1}{(2 \tilde{\imath})^{i+1}}, x \in[0,2 \pi]$. Also, from above it follows that $\widetilde{y}_{k}(x ; z)=\sum_{i=0}^{\infty} c_{k i}(x) z^{i}$, and so, $c_{k i}(x)=\widetilde{w}_{k i}(x) \in$ $\mathbb{R}_{+}$. Finally we obtain that

$$
\left|w_{k i}(x)\right| \leq \widetilde{w}_{k i}(x) \leq \frac{1}{(2 \tilde{l})^{i+1}} \quad \text { for any } \quad x \in[0,2 \pi]
$$


This immediately implies that for any $(t, z) \in \mathbb{D}_{1 / 2} \times \mathbb{D}_{1 / 2}$ and $x \in[0,2 \pi]$ the series determining $Y(x ; z, t, Y(0))$ converges absolutely and uniformly. Thus $Y(x ; z, t, Y(0))$ is continuous in $x$ and holomorphic in $(t, z) \in \mathbb{D}_{1 / 2} \times \mathbb{D}_{1 / 2}$.

The proof of the theorem is complete.

4.4. Proof of Theorem 2.1. Let $I_{k} \subset \mathbb{C}[[t]]$ be the ideal generated by series $f(t)=\sum_{j=k+1}^{\infty} f_{j} t^{j}$. Let $C_{k}:=\mathbb{C}[[t]] / I_{k}$ be the quotient algebra and $\pi_{k}: \mathbb{C}[[t]] \rightarrow C_{k}$ the quotient homomorphism. We identify elements of $C_{k}$ with polynomials $q(\hat{t})=$ $\sum_{i=0}^{k} q_{i} \hat{t}^{i}, \hat{t}:=\pi_{k}(t)$. Let $J_{k} \subset \mathcal{A}(D, L)[[t]$ be the two-sided ideal generated by elements $R(t)=\sum_{j=k+1}^{\infty} r_{j}(D, L, I) t^{j}$ where $r_{j}$ are polynomials in $D, L$ and $I$ with complex coefficients. Let $A_{k}:=\mathcal{A}(D, L)[[t]] / J_{k}$ be the quotient algebra and $\rho_{k}$ : $\mathcal{A}(D, L)[[t]] \rightarrow A_{k}$ the quotient homomorphism. Algebra $A_{k}$ is naturally isomorphic to the algebra $\mathcal{A}(D, L) \otimes_{\mathbb{C}} C_{k}$. Thus we can identify elements of $A_{k}$ with polynomials $Q(\hat{t})=\sum_{j=0}^{k} q_{j}(D, L, I) \hat{t}^{j}$. In particular, for any $R \in A_{k}$ and $f \in \mathbb{C}[[z]]$ the element $R \cdot f$ belongs to $\mathbb{C}[[z]] \otimes_{\mathbb{C}} C_{k}$.

For the fundamental solution $Y(x)$ of (4.5) given by formula (4.8) let us determine $Y_{k}(x):=\rho_{k}(Y(x ; \hat{t}, D, L))=\sum_{i=0}^{k} p_{i}(x ; D, L) \hat{t}^{i}$. Clearly we have

Lemma $4.7 Y(2 \pi) \equiv I$ if and only if $Y_{k}(2 \pi) \equiv I$ for any $k$.

Let

$$
\omega_{k}(x):=\rho_{k}(\omega(x))=\sum_{i=1}^{k} a_{i}(x) \hat{t}^{i} D L^{i-1}
$$

be the image of the form $\omega$ from (4.5). Here $\omega_{k}(x) \in A_{k}$ for any fixed $x$. Let us consider the equation

$$
Y^{\prime}=\omega_{k} \cdot Y
$$

From Picard iteration it follows that the fundamental solution of (4.11) is $Y_{k}(x)$. Then $Y(x ; z, \hat{t}, Y(0))=Y_{k}(x) \cdot Y(0)=\sum_{i=0}^{k} y_{i}(x ; z) \hat{t}^{i}$ is the solution of (4.11) with initial value $Y(0) \in \mathbb{C}[[z]]$. Here $Y(x ; z, \hat{t}, Y(0)) \in \mathbb{C}[[z]] \otimes_{\mathbb{C}} C_{k}$ for any fixed $x$. Let $G\left(A_{k}\right)$ be the group of invertible elements of $A_{k}$. Then monodromy $\rho_{k}: \mathbb{Z} \rightarrow G\left(A_{k}\right)$ of $(4.11)$ is defined by $\rho_{k}(n):=Y_{k}(2 \pi n)$. Our purpose is to prove

Proposition 4.8 Suppose that equation (4.2) determines a center. Then for any $k$ the monodromy $\rho_{k}$ of (4.11) is trivial.

Proof. Let $\mathcal{P}_{s} \subset \mathbb{C}[[z]]$ be the space of complex polynomials of degree at most $s$. Clearly, $D, L: \mathcal{P}_{s} \rightarrow \mathcal{P}_{s-1}$. Below we denote $D_{s}=\left.D\right|_{\mathcal{P}_{s}}, L_{s}=\left.L\right|_{\mathcal{P}_{s}}, I_{s}=\left.I\right|_{\mathcal{P}_{s}}$, and $Y_{k, s}(x):=\left.Y_{k}(x)\right|_{\mathcal{P}_{s}}$. Then

$$
Y_{k, s}(x)=\sum_{i=0}^{k} p_{i}\left(x ; D_{s}, L_{s}\right) \hat{t}^{i} .
$$

Note that $Y_{k, s}(x)$ is the fundamental solution of the equation

$$
Y^{\prime}=\left(\sum_{i=1}^{k} a_{i}(x) \hat{t}^{i} D_{s} L_{s}^{i-1}\right) \cdot Y
$$


The form in this equation is an element of $\mathcal{A}\left(D_{s}, L_{s}\right) \otimes_{\mathbb{C}} C_{k}$. Then the solution of (4.12) with an initial value $Y(0) \in \mathcal{P}_{s}$ is $Y_{k, s}(x) \cdot Y(0)=\sum_{i=0}^{k} y_{i}(x ; z) \hat{t}^{i}$. For any fixed $x$ this solution belongs to $\mathcal{P}_{s} \otimes_{\mathbb{C}} C_{k}$.

Next, recall that the solution $Y(x ; z, t, r)$ of $(4.5)$ with initial value $Y(0)=$ $\sum_{i=0}^{\infty} r^{i+1} z^{i}$ is given by

$$
Y(x ; z, t, r)=Y(x) \cdot Y(0)=\sum_{i=0}^{\infty} y(x ; t, r)^{i+1} z^{i}
$$

where $y(x ; t, r)$ is the solution of (4.2) with initial value $y(0 ; t, r)=r$. Moreover, $y(x ; t, r)=\sum_{i=1}^{\infty} y_{i}(x) t^{i-1} r^{i}$, and the series converges absolutely and uniformly for $x, t \in[0,2 \pi]$ provided that $|r|$ is small enough. In particular, $y(x ; t, r)$ is Lipschitz in $x \in[0,2 \pi]$ and analytic in $t$ and $r$ for $|t|,|r|$ small enough. If equation (4.2) determines a center then $y(2 \pi ; t, r)=r$ for any sufficiently small $t$ and $r$. This implies that $Y(2 \pi ; z, t, r)=Y(0 ; t, z, r)=Y(0)$ as formal power series in $t, r$ and $z$. Let us apply to $Y(x ; z, t, r)$ the homomorphism $\pi_{k}: \mathbb{C}[[t]] \rightarrow C_{k}$. Then we have

$$
\begin{gathered}
Y_{k}(x ; z, \hat{t}, r):=\pi_{k}(Y(x ; z, t, r))=\pi_{k}(Y(x) \cdot Y(0))= \\
\sum_{i=0}^{k} p_{i}(x ; D, L)(Y(0)) \cdot \hat{t}^{i}=\rho_{k}(Y(x)) \cdot Y(0)=Y_{k}(x) \cdot Y(0) .
\end{gathered}
$$

Therefore $Y_{k}(x ; z, \hat{t}, r)=\sum_{i=0}^{\infty}\left(\pi_{k}(y(x ; t, r))\right)^{i+1} z^{i}$ is the solution of (4.11) satisfying $Y_{k}(0 ; z, \hat{t}, r)=Y(0)$. From the center condition we also have $Y_{k}(2 \pi ; z, \hat{t}, r)=Y(0)$. But from the series expansion of $y(x ; t, r)$ it follows that

$$
Y_{k}(x ; z, \hat{t}, r)=\sum_{j=1}^{k+1} \widetilde{y}_{j}(x ; \hat{t}, z) r^{j}
$$

where $\widetilde{y}_{j}(x ; \hat{t}, z) \in C_{k}$ for any fixed $x, z$. In particular, $\widetilde{y}_{j}(2 \pi ; \hat{t}, z)=\widetilde{y}_{j}(0 ; \hat{t}, z)$ for any $j$. Let us now apply to $Y_{k}(x ; z, \hat{t}, r)$ the homomorphism $\pi_{s+1}: \mathbb{C}[[r]] \rightarrow C_{s+1}$. Then we have

$$
\begin{gathered}
Y_{k, s}(x ; z, \hat{t}, \hat{r}):=\pi_{s+1}\left(Y_{k}(x ; z, \hat{t}, r)\right)=\pi_{s+1}\left(Y_{k}(x) \cdot Y(0)\right)= \\
Y_{k}(x) \cdot \pi_{s+1}(Y(0))=Y_{k}(x)\left(\sum_{i=0}^{s} \hat{r}^{i+1} z^{i}\right)=Y_{k, s}(x)\left(\sum_{i=0}^{s} \hat{r}^{i+1} z^{i}\right) .
\end{gathered}
$$

Here $\hat{r}:=\pi_{s+1}(r) \in C_{s+1}$. This means that $Y_{k, s}(x ; z, \hat{t}, \hat{r})$ is the solution of equation (4.12) with the initial value $Y_{s}(0):=\sum_{i=0}^{s} \hat{r}^{i+1} z^{i}$. Moreover, from the center condition we also have $Y_{k, s}(2 \pi ; z, \hat{t}, \hat{r})=Y_{s}(0)$.

Let us identify the element $z^{i-1}$ with the vector $e_{i}=(0, \ldots, 0,1,0, \ldots, 0)$ with 1 at the $i^{\text {th }}$ place $(1 \leq i \leq s+1)$. Then $D_{s}, L_{s}$ can be thought of as nilpotent matrices $A, B$ acting on $\mathbb{C}^{s+1}$. Now we can write (4.12) as

$$
Y^{\prime}=\left(\sum_{i=1}^{k} a_{i}(x) \hat{t}^{i} A B^{i-1}\right) \cdot Y .
$$


Notice that in this identification $Y_{s}(0)$ coincides with the vector $v(\hat{r})=\sum_{i=1}^{s+1} \hat{r}^{i} e_{i}$, the fundamental solution $Y_{k, s}(x)$ of (4.12) coincides with the fundamental solution $Y_{k, s}^{\prime}(x)$ of (4.13), and $Y_{k, s}(x ; z, \hat{t}, \hat{r})$ coincides with the solution $Y_{k, s}(x ; \hat{t}, v(\hat{r}))$ of $(4.13)$ with initial value $v(\hat{r})$. In particular, from the center condition we obtain that

$$
Y_{k, s}(2 \pi ; \hat{t}, v(\hat{r}))=v(\hat{r})
$$

Here we can think of $v(\hat{r})$ as a complex-valued function in variable $\hat{r}$. Let $r_{1}, \ldots, r_{s+1}$ be pairwise distinct complex numbers. By $V\left(r_{1}, \ldots, r_{s+1}\right)$ we denote the matrix whose columns are $v\left(r_{1}\right), \ldots, v\left(r_{s+1}\right)$. Note that $V\left(r_{1}, \ldots, r_{s+1}\right)$ is invertible, because the determinant of this matrix is the Vandermonde determinant. Now from (4.14) we have

$$
V\left(r_{1}, \ldots, r_{s+1}\right)=Y_{k, s}^{\prime}(2 \pi) \cdot V\left(r_{1}, \ldots, r_{s+1}\right) .
$$

This implies that $Y_{k, s}^{\prime}(2 \pi)=Y_{k, s}(2 \pi)=I_{s}$. Thus the monodromy of (4.12) is trivial.

Now, for any $f(z)=\sum_{i=0}^{\infty} c_{i} z^{i} \in \mathbb{C}[[z]]$ we write $f(z)=f_{s}(z)+r_{s}(z)$ where $f_{s}(z)=\sum_{i=0}^{s} c_{i} z^{i}$. Let $Y(x ; \hat{t}, f(z))$ be the solution of (4.11) with initial value $f(z)$. Then we have

$$
\begin{gathered}
Y(2 \pi ; \hat{t}, f(z))-f(z)=\left(Y_{k}(2 \pi)-I\right)(f(z))= \\
\left(Y_{k}(2 \pi)-I\right)\left(f_{s}(z)\right)+\left(Y_{k}(2 \pi)-I\right)\left(r_{s}(z)\right)= \\
\left(Y_{k, s}(2 \pi)-I_{s}\right)\left(f_{s}(z)\right)+\left(Y_{k}(2 \pi)-I\right)\left(r_{s}(z)\right)= \\
\left(Y_{k}(2 \pi)-I\right)\left(r_{s}(z)\right)=\sum_{i=1}^{k} p_{i}(2 \pi ; D, L)\left(r_{s}(z)\right) \cdot \hat{t}^{i} .
\end{gathered}
$$

Since each $p_{i}$ is a homogeneous polynomial of degree $i$ in $D$ and $L$, for $s$ big enough we have

$$
p_{i}(2 \pi ; D, L)\left(r_{s}(z)\right)=\sum_{j=s-k}^{\infty} c_{i j}(f) \cdot z^{j} .
$$

Here $c_{i j}(f) \in \mathbb{C}$ depends on $f$ and $p_{i}$ only. As before, below $I_{l} \subset \mathbb{C}[[z]]$ denotes the ideal generated by elements $f(z)=\sum_{j=l+1}^{\infty} f_{j} z^{j}$. Now the above arguments imply that

$$
Y(2 \pi ; \hat{t}, f(z))-f(z) \in I_{s-k-1} \otimes_{\mathbb{C}} C_{k} \text { for any } s .
$$

Finally, using the fact that $\cap_{j=1}^{\infty} I_{j}=0$ we obtain that $Y(2 \pi ; \hat{t}, f(z))=f(z)$ for any $f(z) \in \mathbb{C}[[z]]$. This implies that $p_{i}(2 \pi ; D, L)(f(z))=0$ for any $f(z) \in \mathbb{C}[[z]]$ and any $1 \leq i \leq k$. Then from the arguments used in the proof of Proposition 4.5 it follows that $p_{i}(2 \pi ; D, L)=0$ as an element of $\mathcal{A}_{0}(D, L)$. In particular, $Y_{k}(2 \pi)=I$.

This completes the proof of the proposition.

From Proposition 4.8 and Lemma 4.7 we conclude that if the original equation (4.2) determines a center then the monodromy $\rho^{\prime}$ of (4.5) is trivial.

Conversely, suppose that the monodromy $\rho^{\prime}$ of equation (4.5) is trivial. Let us consider the solution of this equation with the initial value $Y(0)=\sum_{i=0}^{\infty} r^{i+1} z^{i}$. Then this solution is $Y(x) \cdot Y(0)=\sum_{i=0}^{\infty} y(x ; t, r)^{i+1} z^{i}$ where $Y(x)$ is the fundamental 
solution of $(4.5)$ and $y(x ; t, r)$ is the solution of $(4.2)$ with $y(0 ; t, r)=r$. Also, for $|r|<\frac{e^{-2 \pi}}{2 \tilde{l}}$ and $|t|<\frac{1}{2}$ from Theorem 4.6 it follows that $y(x ; t, r)$ is a continuous function in $x$ analytic in $t$. Now, from the identity for formal power series $Y(2 \pi)=I$ we obtain that $y(2 \pi ; t, r)=r$ for any $r$ and $t$ small enough.

This completes the proof of the theorem.

4.5. Proof of Theorem 2.2. Recall that according to formula (4.8) the fundamental solution of $(4.5)$ is $Y(x):=\sum_{n=0}^{\infty} p_{n}(x ; D, L) t^{n}$, where $p_{0}=I$ and $p_{n}(x ; D, L)=$ $\sum_{i_{1}+\ldots+i_{k}=n} I_{i_{1}, \ldots, i_{k}}(x)\left(D L^{i_{k}-1}\right) \cdots\left(D L^{i_{1}-1}\right)$. Now, the solution of (4.5) with the initial value $\sum_{i=0}^{\infty} r^{i+1} z^{i}$ is $Y(x) \cdot\left(\sum_{i=0}^{\infty} r^{i+1} z^{i}\right)=\sum_{i=0}^{\infty} y(x ; t, r)^{i+1} z^{i}$ where $y(x ; t, r)$ is the solution of $(4.2)$ with $y(0 ; t, r)=r$. Also, the triviality of the monodromy $\rho$ is equivalent to the equation $y(2 \pi ; t, r)-r=0$. This means that in order to find equations determining the center set for $(1.1)$ we should apply each $p_{n}(2 \pi ; D, L)$ to $\sum_{i=0}^{\infty} r^{i+1} z^{i}$, then substitute $z=0$ and equate the latter to 0 . Now, it is clear that we obtain the same equations if we apply $p_{n}(2 \pi ; D, L)$ to $z^{n}$. From here and (4.8) we obtain for $i_{1}+\ldots+i_{k}=n$,

$$
c_{i_{1}, \ldots, i_{k}}:=\left(D L^{i_{k}-1}\right) \cdots\left(D L^{i_{1}-1}\right)\left(z^{n}\right)=\left(n-i_{1}+1\right)\left(n-i_{1}-i_{2}+1\right) \cdots 1 .
$$

Also, the expression $P(r):=y(2 \pi ; 1, r)$ is the first return map for equation (1.1). From above it follows that

$$
P(r)=r+\sum_{n=1}^{\infty} c_{n} r^{n+1} \quad \text { where } \quad c_{n}=\sum_{i_{1}+\ldots+i_{k}=n} c_{i_{1}, \ldots, i_{k}} I_{i_{1}, \ldots, i_{k}} .
$$

This proves the required result.

4.6. Proof of Corollary 2.3. The proof of part (a) follows directly from the above formula for the first return map. Let us prove part (b).

By $\pi_{k}: X \rightarrow \prod_{j=1}^{k} X_{j}$ we denote the natural projection to the first $k$ coordinates. According to part (a) the set $\mathcal{C}_{n}$ is given by equations $I_{k}(a)=-f_{k}(a), 1 \leq k \leq n$, where $I_{k}$ depends on $a_{k}$ and $f_{k}$ depends on $a_{1}, \ldots, a_{k-1}$. Let $\widetilde{\mathcal{C}}_{n}$ be the subset of $\prod_{j=1}^{n} X_{j}$ defined by these equations. Then clearly $\mathcal{C}_{n}=\pi_{n}^{-1}\left(\widetilde{\mathcal{C}}_{n}\right)$. Thus in order to prove the corollary it suffices to prove that $\widetilde{\mathcal{C}}_{n} \subset \prod_{j=1}^{n} X_{j}$ is a closed complex submanifold of codimension $n$ containing 0 . We prove this by induction on $n$.

If $n=1$, then $\widetilde{\mathcal{C}}_{1}$ is determined by the equation $I_{1}(a):=\int_{0}^{2 \pi} a_{1}(s) d s=0$. Since $I_{1}$ is a continuous linear functional, $\widetilde{\mathcal{C}}_{1}$ coincides with the complex hyperplane $\operatorname{Ker}\left(I_{1}\right) \subset X_{1}$. Thus the required statement holds. Suppose now that the statement is proved for $k-1$, let us prove it for $k$. It is easy to see that $\widetilde{\mathcal{C}}_{k}$ is a subset of $\widetilde{\mathcal{C}}_{k-1} \times X_{k}$ determined by the equation $I_{k}(a)=-f_{k}(a)$. Since $\left.I_{k}\right|_{X_{k}}$ is a continuous linear functional, we can decompose $X_{k}=E_{k} \oplus l_{k}$, where $E_{k}=\operatorname{Ker}\left(\left.I_{k}\right|_{X_{k}}\right)$ and $l_{k}$ is the one-dimensional vector space generated by a vector $e_{k}$ such that $I_{k}\left(e_{k}\right)=1$. Further, let us consider the set $R_{k}:=\widetilde{\mathcal{C}}_{k-1} \times E_{k}$. It is clear that $R_{k} \subset \operatorname{Ker}\left(I_{k}\right)$ and $\widetilde{\mathcal{C}}_{k-1} \times X_{k}=R_{k} \oplus l_{k}$. In particular, for any $a \in \widetilde{\mathcal{C}}_{k-1} \times X_{k}$ we have $a=w+v e_{k}$ for some $w \in R_{k}, v \in \mathbb{C}$. Thus in the definition of $\widetilde{\mathcal{C}}_{k}$ we have

$$
v=I_{k}(a)=-f_{k}(a)=-f_{k}(w) .
$$

This shows that $\widetilde{\mathcal{C}_{k}}$ is the graph of the function $-f_{k}: R_{k} \rightarrow \mathbb{C}$. But according to the induction hypothesis, $R_{k} \subset\left(\prod_{j=1}^{k-1} X_{j}\right) \times E_{k}$ is a closed complex submanifold 
of codimension $(k-1)$ containing 0 . From here it follows that $\widetilde{\mathcal{C}}_{k} \subset \widetilde{\mathcal{C}}_{k-1} \times X_{k}$ is a closed complex submanifold of codimension 1 containing 0 . This implies the required result and completes the proof of part (b).

Let us prove part (c).

It follows from the definition that the linear term of the Taylor expansion of $c_{n}$ at $0 \in X$ equals $I_{n}$. It remains to note that the tangent space to $\mathcal{C}_{n}$ at $0 \in X$ is determined as the set of common zeros of the linear terms of $c_{1}, \ldots, c_{n}$, that is, it is given by equations $I_{1}(a)=\ldots=I_{n}(a)=0$.

4.7. Proof of Theorem 2.4. Let $v(x ; r ; a), x \in[0,2 \pi]$, be the Lipschitz solution of equation (1.1) corresponding to $a=\left(a_{1}, a_{2}, \ldots\right) \in X$ with initial value $v(0 ; r ; a)=r$. From the Picard method of successive approximations it follows that

$$
v(x ; r ; a)=r+\sum_{i=1}^{\infty} v_{i}(x ; a) r^{i+1}
$$

where each $v_{i}(x ; a)$ is a Lipschitz function on $[0,2 \pi]$ and the series converges uniformly in the domain $0 \leq x \leq 2 \pi,|r| \leq \widetilde{r}$ for a sufficiently small positive $\tilde{r}$. Assuming that $a \in \mathcal{C}$ we get $v_{i}(0 ; a)=v_{i}(2 \pi ; a)=0$ for any $i$. Now the inverse function theorem implies that there is a function

$$
u(x ; \rho ; a):=\rho+\sum_{i=1}^{\infty} u_{i}(x ; a) \rho^{i+1}
$$

where each $u_{i}(x ; a)$ is a $2 \pi$-periodic Lipschitz function, $u_{i}(0 ; a)=0$, and the series converges uniformly in the domain $-\infty<x<\infty,|\rho| \leq \widetilde{\rho}$ for a sufficiently small positive $\widetilde{\rho}$, such that

$$
u(x ; v(x ; r ; a) ; a) \equiv r
$$

for all sufficiently small $r$. Differentiating this equation in $x$ and then expressing $v^{\prime}(x ; r ; a)$ we obtain

$$
v^{\prime}(x ; r ; a)=-\frac{\sum_{k=1}^{\infty} u_{k}^{\prime}(x ; a) v(x ; r ; a)^{k+1}}{1+\sum_{k=1}^{\infty}(k+1) u_{k}(x ; a) v(x ; r ; a)^{k}} .
$$

This and the equality

$$
v^{\prime}(x ; r ; a)=\sum_{i=1}^{\infty} a_{i}(x) v(x ; r ; a)^{i+1}
$$

imply the required identity of power series.

Conversely, from the identity of Theorem 2.4 arguing in the reverse order we can find a formal solution of (1.1) corresponding to $a \in X$ of the form (4.15) where $v_{i}$ are polynomials in $u_{1}, \ldots, u_{i}, v_{1}, \ldots, v_{i-1}$ without constant terms. In particular, each $v_{i}$ is a $2 \pi$-periodic Lipschitz function equals 0 at 0 . But since $a \in X$, there is also a usual solution of the corresponding equation (1.1) given by (4.15). Hence these two solutions must coincide. This implies the identity $v(0 ; r ; a)=v(2 \pi ; r ; a)=r$ for any sufficiently small $r$.

The proof of the theorem is complete. 


\section{Proofs of Results of Sections 2.2 and 2.3.}

In this section we prove Corollary 2.5 and Propositions 2.6 and 2.7.

Proof of Corollary 2.5. The proof follows directly from the factorization $\rho=\phi \circ \widetilde{\rho}$.

Proof of Proposition 2.6. We set $R_{i}:=t^{i} X_{1} X_{2}^{i-1}$. Let $\mathcal{A}$ be the algebra of polynomials in $X_{1}, X_{2}, \ldots$ with complex coefficients. The main point of the proof is the following

Lemma 5.1 Suppose $P\left(R_{1}, \ldots, R_{k}\right)=\sum_{1 \leq k \leq n} a_{i_{1}, \ldots i_{k}} R_{i_{1}} \cdots R_{i_{k}} \in \mathcal{A}$ is zero. Then all $a_{i_{1}, \ldots i_{k}}$ are zeros.

Proof. The maximal integer $l=\sum_{s=1}^{k} i_{s}$ for indices in the expansion of $P$ will be called the degree of $P$. We prove the lemma by induction on $l$.

For $l=1$ we have $P=c_{1} R_{1}$ and the statement is obvious. Suppose that we already proved the lemma for $l \leq s$, let us prove it for $l=s+1$. We can write $P=P_{1}+\ldots+P_{k}$ where $P_{i}$ contains all terms of $P$ with $R_{i}$ on the right. Since $X_{1}, X_{2}$ are free non-commutative variables, we have $P_{1}=\ldots=P_{k}=0$. Now $P_{i}=Q_{i} \cdot R_{i}$ where $Q_{i}$ is a polynomial of degree $\leq s$ satisfying the hypothesis of the lemma. Thus by the induction hypothesis all coefficients of every $P_{i}$ are zeros.

Further, it follows from formula (2.5) for the fundamental solution $F(x)$ of equation (2.3) that $F(2 \pi)=\sum_{i=0}^{\infty} q_{i}\left(2 \pi, X_{1}, X_{2}\right)$ where $q_{0}=I$ and others $q_{i}$ are polynomials in $R_{1}, R_{2}, \ldots$ of degree $i$ (as in Lemma 5.1). Moreover, every iterated integral

$$
\int \cdots \int_{0 \leq s_{1} \leq \cdots \leq s_{k} \leq 2 \pi} a_{i_{k}}\left(s_{k}\right) \cdots a_{i_{1}}\left(s_{1}\right) d s_{k} \cdots d s_{1}, \quad \sum_{s=1}^{k} i_{s}=i,
$$

is a coefficient before one of monomials of $q_{i}$. Now, the triviality of the monodromy is equivalent to the fact that all $q_{i} \in \mathcal{A}, i \geq 1$, are zeros. According to Lemma 5.1 this, in turn, is equivalent to the fact that all coefficients of all $q_{i}, i \geq 1$, are zeros.

This completes the proof of the proposition.

Proof of Proposition 2.7. The proof of the proposition repeats word-for-word the proof of Proposition 2.6. We leave the details to the reader.

\section{Proof of Theorem 2.9.}

6.1. We refer to the book of Hirzebruch [Hi] for an exposition about fibre bundles.

Let $U \subset \mathbb{C}^{n}$ be a domain containing $\widehat{\Gamma}_{n}$. Since $\widehat{\Gamma}_{n}$ is polynomially convex, for any domain $V \supset \widehat{\Gamma}_{n}$ there is a Stein domain $V^{\prime}$ such that $\widehat{\Gamma}_{n} \subset V^{\prime} \subset V$. Thus without loss of generality we may assume that $U$ is Stein. Let $\pi_{1}(U)$ be the fundamental group of $U$. The universal covering $p: \widetilde{U} \rightarrow U$ is a discrete bundle on $U$ with the fibre $\pi_{1}(U)$. It is defined on an open acyclic cover $\mathcal{U}=\left(U_{i}\right)$ of $U$ by a locally constant cocycle $\left\{c_{i j}\right\} \in Z^{1}\left(\mathcal{U}, \pi_{1}(U)\right)$. By definition, $c_{i j}: U_{i} \cap U_{j} \rightarrow \pi_{1}(U)$ is a constant map such that $c_{i j} c_{j k}=c_{i k}$ on $U_{i} \cap U_{j} \cap U_{k}$. Then $\widetilde{U}$ is biholomorphic to the quotient space of $\sqcup_{j} U_{j} \times \pi_{1}(U)$ by the equivalence relation:

$$
U_{j} \times \pi_{1}(U) \ni z \times g \sim z \times g \cdot c_{i j}^{-1} \in U_{i} \times \pi_{1}(U) .
$$


Let $l_{U}:=l_{2}\left(\pi_{1}(U)\right)$ be the Hilbert space of complex-valued sequences on $\pi_{1}(U)$ with the $l_{2}$-norm. By $L\left(l_{U}\right)$ we denote the Banach space of bounded complex linear operators $l_{U} \rightarrow l_{U}$, and by $G L\left(l_{U}\right)$ the group of invertible operators from $L\left(l_{U}\right)$. Let us define the homomorphism $\xi: \pi_{1}(U) \rightarrow I s o\left(l_{U}\right)$ by

$$
\xi(g)(v)(x):=v\left(x \cdot g^{-1}\right), \quad g, x \in \pi_{1}(U), v \in l_{U} .
$$

Here $I s o\left(l_{U}\right) \subset G L\left(l_{U}\right)$ is the subgroup of unitary operators. It is clear that $\xi$ is a faithful representation, that is, $\operatorname{Ker}(\xi)=\{1\}$.

Next, let us construct the holomorphic Banach vector bundle $E_{\xi}$ on $U$ with fibre $l_{U}$ associated with $\xi$. It is defined as the quotient of $\sqcup_{j} U_{j} \times l_{U}$ by the equivalence relation

$$
U_{j} \times l_{U} \ni x \times w \sim x \times \xi\left(c_{i j}\right)(w) \in U_{i} \times l_{U} .
$$

Since $l_{U}$ is an infinite dimensional Hilbert space, the group $G L\left(l_{U}\right)$ is contractible (see $[\mathrm{K}]$ ). In particular, $E_{\xi}$ is a topologically trivial Banach vector bundle. Now according to the result of Bungart [B] applied to the Stein manifold $U$ we obtain that $E_{\xi}$ is a holomorphically trivial Banach vector bundle on $U$. This means that there is a family of holomorphic functions $F_{i}: U_{i} \rightarrow G L\left(l_{U}\right)$ satisfying

$$
F_{i}^{-1}(z) \cdot F_{j}(z)=\xi\left(c_{i j}\right) \quad \text { on } \quad U_{i} \cap U_{j} .
$$

Note that cocycle $\left\{\xi\left(c_{i j}\right)\right\}$ is locally constant, i.e. $d\left(\xi\left(c_{i j}\right)\right)=0$ for any $i, j$. Then we define the global holomorphic 1 -form $\omega$ on $X$ with values in $L\left(l_{U}\right)$ by the formula

$$
\left.\omega\right|_{U_{i}}:=d F_{i} \cdot F_{i}^{-1} .
$$

Clearly $\omega$ satisfies the Frobenius condition $d \omega-\omega \wedge \omega=0$, which is equivalent to the fact that equation

$$
d F=\omega \cdot F
$$

is locally solvable on $U$. Let $p^{*} \omega$ be the pullback of $\omega$ to $\widetilde{U}$. Consider the pullback to $\widetilde{U}$ of $(6.1)$

$$
d F=p^{*} \omega \cdot F .
$$

Then from simply connectedness of $\widetilde{U}$ it follows that (6.2) has a global holomorphic solution $F: \widetilde{U} \rightarrow G L\left(l_{U}\right)$. On the connected component $U_{i} \times\{g\}$ of the open set $p^{-1}\left(U_{i}\right):=\sqcup_{g \in \pi_{1}(U)} U_{i} \times\{g\} \subset \widetilde{U}$ the solution $F$ equals $p^{*} F_{i} \cdot \xi\left(g^{-1}\right)$.

Another way to obtain a solution of (6.2) is by using Picard iteration. To this end, we fix a point $z_{0} \in \widetilde{U}$ and for any piecewise smooth path $\gamma$ joining $z_{0}$ with a point $z$ we set

$$
S(z)=I+\sum_{i=1}^{\infty} \int \cdots \int_{\gamma} \omega \cdots \omega .
$$

The $k$-th term of this series is the $k$-iterated integral of $\omega$ over $\gamma$ defined similarly to (2.5). It is well known (cf. [Na]) that the series converges uniformly on compacts in $\widetilde{U}$ to a $G L\left(l_{U}\right)$-valued function $S$ satisfying (6.2), and $S\left(z_{0}\right)=I$. Therefore 
$S(z)=F(z) \cdot F^{-1}\left(z_{0}\right)$ where $F$ is the solution of (6.2) constructed above. Moreover, by the definition of $F$,

$$
S(g z)=S(z) \cdot \xi\left(g^{-1}\right) \quad \text { for any } \quad g \in \pi_{1}(U), z \in \widetilde{U} .
$$

In particular, for $z=z_{0}$ we have $S\left(g z_{0}\right)=\xi\left(g^{-1}\right)$.

Let $\gamma_{g} \subset U$ be a loop based at $p\left(z_{0}\right)$ which represents an element $g \in \pi_{1}\left(U, p\left(z_{0}\right)\right)$. Then the path on $\widetilde{U}$ with the origin at $z_{0}$ and the endpoint at $g z_{0}$ represents the lifting of $\gamma_{g}$ to $\widetilde{U}$. Now the correspondence $\gamma_{g} \mapsto \xi\left(g^{-1}\right)$ determines a homomorphism $\widetilde{\xi}: \pi_{1}\left(U, p\left(z_{0}\right)\right) \rightarrow \operatorname{Aut}\left(G L\left(l_{U}\right)\right)$ defined by

$$
\widetilde{\xi}(g)(H)=H \cdot \xi\left(g^{-1}\right), \quad g \in \pi_{1}\left(U, p\left(z_{0}\right)\right), H \in G L\left(l_{U}\right) .
$$

By the definition of $\xi$ we obtain that $\operatorname{Ker}(\widetilde{\xi})=\{1\}$.

6.2. For basic facts of complex analysis in domains of holomorphy see, e.g. the book of Henkin and Leiterer [HL].

Let us write the form $\omega$ in (6.1) as

$$
\omega(z)=\sum_{j=1}^{n} R_{j}(z) d z_{j}
$$

where $z_{1}, \ldots, z_{n}$ are standard coordinates on $\mathbb{C}^{n}$, and $R_{1}, \ldots, R_{n}$ are holomorphic $L\left(l_{U}\right)$-valued functions on $U$. Let $V \subset \subset U$ be a domain containing $\widehat{\Gamma}$. Then there is a Weil polynomial polyhedron

$$
D=\left\{z \in U:\left|P_{k}(z)\right|<1, P_{k} \in \mathbb{C}\left[z_{1}, \ldots, z_{n}\right], k=1, \ldots, N\right\} \subset V
$$

containing $\widehat{\Gamma}_{n}$. Note that for $L\left(l_{U}\right)$-valued holomorphic functions defined in an open neighbourhood of $V$ we have exactly the same integral representations as for scalar holomorphic functions. In particular, any such function $f$ inside $D$ can be represented as a finite sum of holomorphic $L\left(l_{U}\right)$-valued functions $f_{J}$ such that each

$$
f_{J}(z)=\int_{\sigma_{J}} f(\xi) K_{J}(\xi, z) \eta(\xi)
$$

where $\eta(\xi)=d \xi_{1} \wedge \ldots d \xi_{n}, K_{J}(\xi, z)$ is a rational holomorphic function in $z$ and $\xi$, and $\sigma_{J}$ is an $n$-dimensional part of the Silov boundary of $D$. Moreover, one can uniformly approximate on compacts in $D$ each $K_{J}(\xi, z)$ by holomorphic functions $K_{J i}(\xi, z), i=1, \ldots$, which are holomorphic polynomials in $z$. In particular, we obtain the Oka-Weil approximation result.

Proposition 6.1 Any $L\left(l_{U}\right)$-valued function holomorphic in an open neighbourhood $V \subset \subset U$ of $\widehat{\Gamma}_{n}$ can be uniformly approximated on $\widehat{\Gamma}_{n}$ by $L\left(l_{U}\right)$-valued holomorphic polynomials.

From this proposition it follows that there is a sequence of polynomial 1-forms

$$
\omega_{i}(z)=\sum_{j=1}^{n} R_{j i}(z) d z_{j}
$$


such that $R_{j i}(z)=\sum_{0 \leq|\alpha| \leq d_{j i}} R_{j i, \alpha} z^{\alpha}, R_{j i, \alpha} \in L\left(l_{U}\right)$, and

$$
\lim _{i \rightarrow \infty} \sup _{z \in \widehat{\Gamma}}\left\|R_{j}(z)-R_{j i}(z)\right\|=0 .
$$

Here $z^{\alpha}:=z^{\alpha_{1}} \cdots z^{\alpha_{n}}$ for any $\alpha:=\left(\alpha_{1}, \ldots, \alpha_{n}\right) \in\left(\mathbb{Z}_{+}\right)^{n},|\alpha|:=\sum_{i=1}^{n} \alpha_{i}$, and $\|\cdot\|$ denotes the Banach norm on $L\left(l_{U}\right)$.

6.3. Next, we recall the Ree formula $[\mathrm{R}]$ for the product of iterated integrals.

A permutation $\sigma$ of $\{1,2, \ldots, r+s\}$ is a shuffle of type $(r, s)$ if

$$
\sigma^{-1}(r+1)<\sigma^{-1}(2)<\cdots<\sigma^{-1}(r)
$$

and

$$
\sigma^{-1}(r+1)<\sigma^{-1}(r+2)<\cdots<\sigma^{-1}(r+s) .
$$

Let $f_{1}, f_{2}, \ldots, f_{r+s} \in L^{\infty}([0, t])$. As before, we set

$$
I_{i_{1}, \ldots, i_{k}}(x):=\int \cdots \int_{0 \leq s_{1} \leq \cdots \leq s_{k} \leq x} f_{i_{k}}\left(s_{k}\right) \cdots f_{i_{1}}\left(s_{1}\right) d s_{k} \cdots d s_{1}
$$

Then

$$
I_{1,2, \ldots, r}(x) \cdot I_{r+1, r+2, \ldots, r+s}(x)=\sum_{\sigma} I_{\sigma(1), \sigma(2), \ldots, \sigma(r+s)}(x)
$$

where $\sigma$ runs over the shuffles of type $(r, s)$.

Going back to the proof of the theorem we set $\widetilde{R}_{j}(x):=A_{n}^{*}\left(R_{j}\right)(x), j=1, \ldots, n$. Then $\widetilde{R}_{j}$ are $L\left(l_{U}\right)$-valued continuous functions on $S^{1}$.

Lemma 6.2 For any positive integers $k$ and $i_{1}, \ldots, i_{k}, 1 \leq i_{s} \leq n$, we have

$$
\int \cdots \int_{0 \leq s_{1} \leq \cdots \leq s_{k} \leq 2 \pi} \widetilde{R}_{i_{1}}\left(s_{k}\right) a_{i_{1}}\left(s_{k}\right) \cdots \widetilde{R}_{i_{k}}\left(s_{1}\right) a_{i_{k}}\left(s_{1}\right) d s_{k} \cdots d s_{1}=0 .
$$

Proof. According to Proposition 6.1 it suffices to prove the result for $\widetilde{R}_{j}$ holomorphic $L\left(l_{U}\right)$-valued polynomials. Moreover, in this case it suffices to prove the lemma for scalar monomials. Thus without loss of generality we may assume that

$$
\widetilde{R}_{i_{l}}\left(s_{l}\right)=\widetilde{a}_{1}\left(s_{l}\right)^{\alpha_{1, i_{l}}} \ldots \widetilde{a}_{n}\left(s_{l}\right)^{\alpha_{n, i_{l}}} .
$$

Here $\widetilde{a}_{i}$ is the antiderivative of $a_{i}$. Now the required result follows from the fact that by the Ree formula each integral on the left-hand-side of (6.4) can be written as a finite sum of integrals of the form

$$
\int \cdots \int_{0 \leq s_{1} \leq \cdots \leq s_{r} \leq 2 \pi} a_{j_{r}}\left(s_{r}\right) \cdots a_{j_{1}}\left(s_{1}\right) d s_{r} \cdots d s_{1}
$$

where $r=\sum_{k=1}^{n}\left(\alpha_{k, i_{l}}+1\right)$, and from equations (2.7). The calculation is straightforward and we leave it as an exercise for the readers.

6.4. Let us finish the proof of the theorem.

Let $A_{n}: S^{1} \rightarrow U$ represent an element $h \in \pi_{1}\left(U, p\left(z_{0}\right)\right)$. Let $\widetilde{A}_{n}: S^{1} \rightarrow \widetilde{U}$ be the lifting of $A_{n}$ with the origin at $z_{0}$. Then its endpoint is $h z_{0}$. Let $S(z)$ be the 
solution of (6.2) from Section 4.1. Then $S\left(h z_{0}\right)=\xi\left(h^{-1}\right)$. On the other hand, this value can be obtained by formula (6.3) where the integrals are taken over the path $\widetilde{A}_{n}: S^{1} \rightarrow \widetilde{U}$. To calculate these integrals we take the pullback of the form $p^{*} \omega$ by $\widetilde{A}_{n}$, and then calculate usual iterated integrals of $A_{n}^{*} \omega:=\widetilde{A}_{n}^{*}\left(p^{*} \omega\right)$ over $[0,2 \pi]$. But each of such iterated integrals is zero by Lemma 6.2. This means that $\xi\left(h^{-1}\right)=I$. Because $\xi$ is a faithful representation the latter implies that $h=1 \in \pi_{1}\left(U, p\left(z_{0}\right)\right)$.

The proof of the theorem is complete.

\section{Proofs of Theorem 2.12 and Corollaries 2.10, 2.15 and 2.17 .}

Proof of Corollary 2.10. Suppose $\Gamma_{n}$ is triangulable, $\widehat{\Gamma}_{n}=\Gamma_{n}$, and $\Gamma_{n}$ satisfies the hypothesis of Theorem 2.9. From the triangulability of $\Gamma_{n}$ it follows that $\Gamma_{n}$ is homeomorphic to a one-dimensional simplicial complex (i.e., a finite graph). Moreover, by Borsuk's theorem (for the references see, e.g. [Hu]) there is an open connected neighbourhood $U$ of $\Gamma_{n}$ and a retraction $r: U \rightarrow \Gamma_{n}$. Let $i: \Gamma_{n} \hookrightarrow U$ be the embedding. By $i_{*}: \pi_{1}\left(\Gamma_{n}\right) \rightarrow \pi_{1}(U)$ and $r_{*}: \pi_{1}(U) \rightarrow \pi_{1}\left(\Gamma_{n}\right)$ we denote the corresponding induced homomorphisms of fundamental groups. Then $r_{*} \circ i_{*}: \pi_{1}\left(\Gamma_{n}\right) \rightarrow \pi_{1}\left(\Gamma_{n}\right)$ is the identity homomorphism. Now, it follows from the condition $\widehat{\Gamma}_{n}=\Gamma_{n}$ and Theorem 2.9 that the path $i \circ A_{n}: S^{1} \rightarrow U$ represents $1 \in \pi_{1}(U)$. Thus from above we obtain that $A_{n}: S^{1} \rightarrow \Gamma_{n}$ represents $1 \in \pi_{1}\left(\Gamma_{n}\right)$. This means that $A_{n}$ is contractible to a point inside $\Gamma_{n}$.

Further, let $p: \Gamma_{n u} \rightarrow \Gamma_{n}$ be the universal covering of $\Gamma_{n}$. Then $\Gamma_{n u}$ can be thought of as an infinite tree. The group $\pi_{1}\left(\Gamma_{n}\right)$ (which is a free group with a finite number of generators) acts discretely on $\Gamma_{n u}$. According to the covering homotopy theorem (see, e.g. [Hu, Ch.III]), there is a map $A_{2 n}: S^{1} \rightarrow \Gamma_{n u}$ such that $A_{n}=$ $p \circ A_{2 n}$. The image $G_{n}:=A_{2 n}\left(S^{1}\right) \subset \Gamma_{n u}$ is a connected compact subset. Therefore $G_{n}$ is homeomorphic to a finite tree. Finally, we set $A_{1 n}:=\left.p\right|_{G_{n}}$.

Conversely, if $A_{n}$ admits the factorization of Corollary 2.10 then $A_{2 n}: S^{1} \rightarrow G_{n}$ is contractible to a point inside $G_{n}$. Therefore $A_{n}: S^{1} \rightarrow \Gamma_{n}$ is contractible to a point inside $\Gamma_{n}$.

Proof of Theorem 2.12. Suppose $\Gamma_{n}$ is Lipschitz triangulable, $\widehat{\Gamma}_{n}=\Gamma_{n}$, and $A_{n}: S^{1} \rightarrow \Gamma_{n}$ is contractible in $\Gamma_{n}$ to a point. By the covering homotopy theorem, there is a covering $s: \widetilde{U} \rightarrow U$ and a Lipschitz embedding $i: \Gamma_{n u} \hookrightarrow \widetilde{U}$ such that $p=s \circ i$. Without loss of generality we may consider $U$ as a submanifold of some $\mathbb{R}^{N_{n}}$ so that $i\left(\Gamma_{n u}\right) \subset \mathbb{R}^{N_{n}}$ has an exhaustion by Lipschitz triangulable compact subsets. It follows from the Whitney embedding theorem. We identify $\Gamma_{n u}$ with $i\left(\Gamma_{n u}\right)$.

Now, the contractibility of $A_{n}$ implies that there is a map $A_{2 n}: S^{1} \rightarrow \Gamma_{n u} \subset \mathbb{R}^{N_{n}}$ such that $A_{n}=s \circ A_{2 n}$. Since $A_{n}$ is Lipschitz, the map $A_{2 n}$ is also Lipschitz by the definition of $\widetilde{U}$. Let us consider the equation on $U$

$$
d F=\eta \cdot F, \quad \text { where } \quad \eta:=\sum_{i=1}^{n} t^{i} X_{1} X_{2}^{i-1} d z_{i}
$$


This equation is not locally solvable. However, its lifting $d F=s^{*} \eta \cdot F$ restricted to $\Gamma_{n u}$ is locally solvable. This follows from the fact that $\Gamma_{n u}$ outside a countable discrete set of points is the disjoint union of one-dimensional Lipschitz manifolds $M_{i}, i=1,2, \ldots$ (the consequence of Lipschitz triangulability). Moreover, each $M_{i}$ is the image of the interval $(0,1)$ under some Lipschitz map $g_{i}:(0,1) \rightarrow \mathbb{R}^{N_{n}}$ so that $g_{i}^{-1}: M_{i} \rightarrow(0,1)$ is locally Lipschitz. Now because $\Gamma_{n u}$ is a tree, we can solve the equation $d F=s^{*} \eta \cdot F$ on $\Gamma_{n u}$ by Picard iteration (as in (6.3)). Indeed, since $g_{i}$ is Lipschitz, we can solve the pullback of this equation by $g_{i}$ to $(0,1)$. Then we transfer this solution to $M_{i}$ by $g_{i}^{-1}$ to obtain a solution of $d F=s^{*} \eta \cdot F$ on $M_{i}$. Finally, we apply the Picard method successively on the edges of $\Gamma_{n u}$ to glue the local solutions to a global one. Let us denote this solution by $F$. Then $F(x), x \in \Gamma_{n u}$, is an element of the group $G\left(X_{1}, X_{2}\right)[[t]]$ (see Introduction). Moreover, since $A_{n}^{-1}(x), x \in \Gamma_{n}$, is countable, the coefficients of the series determining $A_{2 n}^{*} F$ are locally Lipschitz on an open subset $O=S^{1} \backslash T$ of $S^{1}$ where $T \subset S^{1}$ is countable. So $A_{2 n}^{*} F$ has the derivative almost everywhere on $S^{1}$. Then clearly

$$
\frac{d\left(A_{2 n}^{*} F\right)}{d x}=\left(A_{2 n}^{*}\left(s^{*} \eta\right)\right) \cdot A_{2 n}^{*} F=\left(\sum_{i=1}^{n} a_{i}(x) t^{i} X_{1} X_{2}^{i-1}\right) \cdot A_{2 n}^{*} F .
$$

Without loss of generality we may assume that $A_{2 n}^{*} F(0)=I$. On the other hand, there is a global solution $H$ of the above equation (which is equation $(2.8)$ ) on $[0,2 \pi$ ) such that $H(0)=I$, and such that coefficients of $H$ in the series expansion are Lipschitz functions. In particular, coefficients in the series expansion of $H^{-1} \cdot A_{2 n}^{*} F$ are locally Lipschitz on $O$. Thus we have

$$
\frac{d\left(H^{-1} \cdot A_{2 n}^{*} F\right)}{d x}=0 \quad \text { almost everywhere on } S^{1} .
$$

Since $H^{-1} \cdot A_{2 n}^{*} F$ is continuous and $\left(H^{-1} \cdot A_{2 n}^{*} F\right)(0)=I$, this and the results of Section 2.1 imply that $H(x)=A_{2 n}^{*} F(x)$ on $[0,2 \pi)$. But $A_{2 n}^{*} F(2 \pi)=A_{2 n}^{*} F(0)$ showing that the monodromy $\widetilde{\rho}_{n}$ of $(2.8)$ is trivial.

The proof of the theorem is complete.

Proof of Corollary 2.15. (A) Let $\nu: \widetilde{X} \rightarrow X$ be the normalization of $X$. Then $\widetilde{X}$ is a non-compact (possibly disconnected) complex Riemann surface. Since $\widetilde{A}_{n}^{-1}(x)$ is finite for any $x \in \widetilde{A}(R)$, the definition of the normalization implies that there is a continuous map $\widetilde{A}_{1 n}: R \rightarrow \widetilde{X}$ such that $\widetilde{A}_{n}=\nu \circ \widetilde{A}_{1 n}$. In particular, the image of $R$ belongs to a connected component of $\widetilde{X}$. Thus $\Gamma_{n}:=A_{n}\left(S^{1}\right)$ belongs to an irreducible component of $X$. In what follows without loss of generality we may assume that $X$ itself is irreducible.

Lemma 7.1 Suppose that $X$ satisfies condition (1) of Corollary 2.15. Then $\widehat{\Gamma}_{n} \subset X$.

Proof. By the definition of $U$ we obtain that $\widehat{\Gamma}_{n}$ belongs to a polynomially convex compact $K_{j}$. Since $X$ is a closed subspace of the Stein domain $U$, it follows (see e.g. [GR, Ch.5, Sect.4]) that $X$ is the set of common zeros of a family $\left\{f_{i}\right\}_{i \in I}$ of holomorphic on $U$ functions. Since $K_{j}$ is polynomially convex, by the OkaWeil approximation theorem each $f_{i}$ can be uniformly approximated in a small 
open neighbourhood $O \subset \subset U$ of $K_{j}$ by holomorphic polynomials. Assume, to the contrary, that there exists a $z \in \widehat{\Gamma}_{n}$ such that $z \notin X \cap K_{j}$. Then there is an index $i \in I$ and an $\epsilon>0$ such that $\left|f_{i}(z)\right|>\epsilon$. Moreover, the polynomial approximation of $f_{i}$ produces a holomorphic polynomial $p_{i}$ such that

$$
\max _{K_{j} \cap X}\left|p_{i}\right| \leq \frac{\epsilon}{2} \quad \text { but } \quad\left|p_{i}(z)\right|>\frac{\epsilon}{2}
$$

This contradicts to the fact that $z \in \widehat{\Gamma}_{n} \subset K_{j} \widehat{\cap} X$.

Next, there is an open connected neighbourhood $Y \subset \subset X$ of the compact connected set $\widehat{\Gamma}_{n} \subset X$. Since $Y$ is an analytic space, by the Lojasiewicz theorem [Lo] it is triangulated. In particular, there is an open connected neighbourhood $O_{Y} \subset \subset U$ of $Y$ and a retraction $r: O_{Y} \rightarrow Y$. Then from the triviality of the monodromy $\widetilde{\rho}_{n}$, as in the proof of Corollary 2.10, we obtain under hypothesis (1) of Corollary 2.15 that the path $A_{n}: S^{1} \rightarrow Y$ is contractible in $Y$ to a point.

Further, for one-dimensional Stein spaces $X$ and $\widetilde{X}$ the normalization map $\nu$ : $\widetilde{X} \rightarrow X$ induces an embedding homomorphism of the fundamental groups. In particular, $\widetilde{A}_{1 n}: S^{1} \rightarrow \widetilde{X}$ is contractible to a point in $\widetilde{X}$. Let $p: \widetilde{X}_{u} \rightarrow \widetilde{X}$ be the universal covering. Then by the covering homotopy theorem there is a covering map $A_{2 n}: S^{1} \rightarrow \widetilde{X}_{u}$ such that $\widetilde{A}_{1 n}=p \circ A_{2 n}$. Since $\widetilde{X}$ is hyperbolic, $\widetilde{X}_{u}$ is conformally equivalent to the unit disk $\mathbb{D}$. Moreover, $A_{2 n}\left(S^{1}\right) \subset \mathbb{D}$ is compact. Therefore we can choose a domain $D \subset \subset \mathbb{D}$ such that $A_{2 n}\left(S_{1}\right) \subset D$ and $D$ is conformally equivalent to $\mathbb{D}$. Now we set $A_{1 n}:=\left.(\nu \circ p)\right|_{D}$.

To finish the first part of the proof let us check that $A_{2 n}$ is locally Lipschitz outside a finite set in $S^{1}$.

Let $X_{s}$ be a finite set of singular points of $X$ containing in $\Gamma_{n}$. By our hypotheses, the preimage of each point of $A_{n}: S^{1} \rightarrow X$ is finite. Thus $Y:=A_{n}^{-1}\left(X_{s}\right) \subset S^{1}$ is finite. Now by the definition of the normalization map, there is an open connected neighbourhood $V \subset X$ of $\Gamma_{n}$ such that $V \backslash X_{s}$ is smooth, and there is the holomorphic inverse map $\nu^{-1}: V \backslash X_{s} \rightarrow \widetilde{X}$. Further, $p: \widetilde{X}_{u} \rightarrow \widetilde{X}$ is a locally biholomorphic map. Finally, the lifted map $A_{2 n}$ outside $Y$ locally is the composite $p^{-1} \circ \nu^{-1} \circ A_{n}$. Since $A_{n}$ is Lipschitz, this implies that $A_{2 n}$ is locally Lipschitz outside $Y$. Note that if $\widetilde{A}_{n}: R \rightarrow X$ is a holomorphic map of an open annulus containing $S^{1}$, then the lifted map $\widetilde{A}_{1 n}: R \rightarrow \widetilde{X}$ is holomorphic, as well. Moreover, since $\widetilde{A}_{1 n}: S^{1} \rightarrow \widetilde{X}$ is contractible to a point, the covering homotopy theorem implies that there is a holomorphic map $A_{2 n}^{\prime}: R \rightarrow \widetilde{X}_{u}$ with $\left.A_{2 n}^{\prime}\right|_{S^{1}}=A_{2 n}$ which covers $\widetilde{A}_{2 n}$. In particular, there is an open annulus $R_{1} \subset R$ containing $S^{1}$ such that $A_{2 n}^{\prime}\left(R_{1}\right) \subset D$. This proves the statement (2) of Remark 2.16.

Conversely, suppose $A_{n}=A_{1 n} \circ A_{2 n}$ where $A_{n}, A_{1 n}$ and $A_{2 n}$ satisfy hypotheses of Corollary 2.15. Let us check that the monodromy $\widetilde{\rho}_{n}$ of the corresponding equation (2.8) is trivial.

As in the proof of Theorem 2.12 we consider equation (7.1) defined on $U$. Its lifting to $\mathbb{D}$ by $A_{1 n}$ is locally solvable, because the lifted form $A_{1 n}^{*} \eta$ is a holomorphic 1 -form on $\mathbb{D}$. Since $\mathbb{D}$ is simply connected, the lifted equation $d F=A_{1 n}^{*} \eta \cdot F$ has a global holomorphic solution $F$ on $U$. (This solution can be obtained by Picard iteration.) Let us consider $A_{2 n}^{*} F$ on $S^{1}$. By the hypothesis, it is continuous, 
locally Lipschitz outside a finite set $Y$. Therefore $A_{2 n}^{*} F$ has the derivative almost everywhere on $S^{1} \backslash Y$ such that

$$
\frac{d\left(A_{2 n}^{*} F\right)}{d x}=\left(A_{2 n}^{*}\left(A_{1 n}^{*} \eta\right)\right) \cdot A_{2 n}^{*} F=\left(\sum_{i=1}^{n} a_{i}(x) t^{i} X_{1} X_{2}^{i-1}\right) \cdot A_{2 n}^{*} F
$$

From here as in the proof of Theorem 2.12 we obtain that $A_{2 n}^{*} F$ is Lipschitz and the monodromy $\widetilde{\rho}_{n}$ of $(2.8)$ is trivial.

The proof of part (1) of the corollary is complete.

(B) Suppose now that $X$ satisfies hypothesis (2). As above there is a lifting $\widetilde{A}_{1 n}: R \rightarrow \widetilde{X}$ of $A_{n}: R \rightarrow X$. We will show that $\widetilde{A}_{1 n}: S^{1} \rightarrow \widetilde{X}$ is contractible to a point. Then the further proof repeats literally the proof presented in $(\mathrm{A})$.

First, note that groups $\pi_{1}(X)$ and $\pi_{1}(\widetilde{X})$ are free (because any one-dimensional Stein space is homotopically equivalent to a one-dimensional CW-complex $[\mathrm{H}]$ ). Then hypothesis $(2)$ implies that $\pi_{1}(X)$ has a finite number of generators. Further, $\nu_{*}: H_{1}(\widetilde{X}, \mathbb{Z}) \rightarrow H_{1}(X, \mathbb{Z})$ is an embedding (because $\nu$ is one-to-one outside a discrete set). This implies that $\pi_{1}(\widetilde{X})$ is finitely generated, as well. Let $\Omega_{p}$ denote the set of holomorphic 1 -forms with polynomial coefficients on $\mathbb{C}^{n}$. By $\nu^{*} \Omega_{p}$ we denote its pullback by $\nu$ to $\widetilde{X}$, and by $\Omega(\widetilde{X})$ the set of all holomorphic 1 -forms on $\widetilde{X}$. Because $\widetilde{X}$ is a Stein manifold, the de Rham 1-cohomology group $H^{1}(\widetilde{X}, \mathbb{C})$ can be computed by $\Omega(\widetilde{X})$. Namely, for each $\delta \in H^{1}(\widetilde{X}, \mathbb{C})$ there is an $\omega \in \Omega(\widetilde{X})$ such that

$$
\delta(\gamma)=\int_{\gamma} \omega \text { for any } \gamma \in H_{1}(\widetilde{X}, \mathbb{C})
$$

Since $\nu_{*}: H_{1}(\widetilde{X}, \mathbb{C}) \rightarrow H_{1}(X, \mathbb{C})$ is an injection and $\operatorname{dim}_{\mathbb{C}} H_{1}(X, \mathbb{C})<\infty$, for any $\delta \in H^{1}(\widetilde{X}, \mathbb{C})$ there is a $\xi \in H^{1}(X, \mathbb{C})$ such that $\nu^{*} \xi=\delta$. In particular, hypothesis (2) implies that each $\delta \in H^{1}(\widetilde{X}, \mathbb{C})$ can be defined by (7.2) with $\omega \in \nu^{*} \Omega_{p}$. Thus for any $\omega \in \Omega(\widetilde{X})$ there is an $\omega^{\prime} \in \nu^{*} \Omega_{p}$ such that $\omega-\omega^{\prime}=d f$ for a holomorphic $f \in \mathcal{O}(\widetilde{X})$. (This means that the embedding $\nu^{*} \Omega_{p} \hookrightarrow \Omega(\widetilde{X})$ is a quasi-isomorphism.) The rest of the proof can be deduced from Sullivan's theory of minimal models of commutative differential graded algebras (see $[\mathrm{Su}]$ ). For the sake of completeness we present the sketch of the proof.

Let $N_{n} \subset G L_{n}(\mathbb{C})$ be the complex Lie subgroup of upper triangular unipotent matrices. By $n_{n} \subset g l_{n}(\mathbb{C})$ we denote the corresponding Lie algebras. For any homomorphism $\rho: \pi_{1}(\widetilde{X}) \rightarrow N_{n}$ by $V_{\rho}$ we denote the flat vector bundle on $\widetilde{X}$ constructed by $\rho$ (for definitions see e.g. [KN, Ch. II]). Since $N_{n}$ is contractible to a point, $V_{\rho}$ is topologically trivial. Further, since $\widetilde{X}$ is Stein, by the Grauert theorem [Gr] $V_{\rho}$ is also holomorphically trivial. Therefore it is determined by a holomorphic flat connection on the trivial bundle $\widetilde{X} \times \mathbb{C}^{n}$, that is, by an $n_{n}$-valued holomorphic 1 -form $\omega$ on $\widetilde{X}$. (Note that $\widetilde{X}$ is one-dimensional and so the Frobenius condition $d \omega-\omega \wedge \omega=0$ is automatically fulfilled.) The main point of the proof is

Lemma 7.2 There are a holomorphic function $F: \widetilde{X} \rightarrow N_{n}$ and a holomorphic $n_{n}$-valued 1-form $\eta$ with entries from $\nu^{*} \Omega_{p}$ such that

$$
F^{-1} \cdot \omega \cdot F-F^{-1} \cdot d F=\eta .
$$


Proof. We can write $\omega=\left(\omega_{k r}\right)$ as $\omega_{1}+\left(\omega-\omega_{1}\right)$ where $\omega_{1}=\left(\omega_{1, k r}\right), \omega_{1, k 1+k}=\omega_{k 1+k}$, $k=1, \ldots, n-1$, and $\omega_{1, k r}=0$ otherwise. According to hypothesis (2) and the above arguments, there is a holomorphic matrix 1-form $\eta_{1}=\left(\eta_{1, k r}\right)$ such that $\eta_{1, k r}=0$ if $r-k \neq 1$, all $\eta_{1, k r} \in \nu^{*} \Omega_{p}$ and $\omega_{1}-\eta_{1}=d f_{1}$, where $f_{1}=\left(f_{1, k r}\right)$ is a holomorphic matrix such that $f_{1, k r}=0$ if $r-k \neq 1$. We set $F_{1}=I_{n}+f_{1}$ where $I_{n} \in G L_{n}(\mathbb{C})$ is the unit matrix. Then

$$
\omega_{1}^{\prime}=F_{1}^{-1} \cdot \omega \cdot F_{1}-F_{1}^{-1} \cdot d F_{1}
$$

is such that $\omega_{1, k 1+k}^{\prime}=\eta_{1, k 1+k} \in \nu^{*} \Omega_{p}$ for any $k$. We will continue this process. Next, we find a holomorphic matrix $f_{2}=\left(f_{2, k r}\right)$ such that $f_{2, k r}=0$ if $r-k \neq 2$, and $\omega_{1}^{\prime}-d f_{2}$ has two diagonals after the main one which consist of elements from $\nu^{*} \Omega_{p}$. Thus for $F_{2}=I_{n}+f_{2}$ we have that

$$
\omega_{2}^{\prime}=F_{2}^{-1} \cdot \omega_{1}^{\prime} \cdot F_{2}-F_{2}^{-1} \cdot d F_{2}
$$

has two diagonals after the main one with entries from $\nu^{*} \Omega_{p}$ etc. After $n-1$ steps we obtain the required matrix $\eta:=\omega_{n-1}^{\prime}$ with entries from $\nu^{*} \Omega_{p}$. It remains to set $F:=F_{1} \cdot F_{2} \cdots F_{n-1}$.

Let $\widetilde{\rho}: \pi_{1}(\widetilde{X}) \rightarrow N_{n}$ be the representation constructed by the flat connection $\eta$. Since by Lemma 7.2 connections $\eta$ and $\omega$ are $d$-gauge equivalent, representations $\rho$ and $\widetilde{\rho}$ are conjugate (see also, e.g. [O, Sec. 4,5]). Namely, there is a matrix $C \in N_{n}$ such that $C^{-1} \cdot \rho \cdot C=\widetilde{\rho}$. In particular, $\operatorname{Ker}(\rho)=\operatorname{Ker}(\widetilde{\rho})$. Now as in the proof of Theorem 2.9 we obtain that if the monodromy of equation (2.8) is trivial the element $\gamma$ representing the path $\widetilde{A}_{1 n}: S^{1} \rightarrow \widetilde{X}$ belongs to $\operatorname{Ker}(\widetilde{\rho})$ (because $\widetilde{\rho}$ is defined by Picard iteration applied to $\eta$ ). Let $G$ be the intersection of kernels of all homomorphisms $\rho: \pi_{1}(\widetilde{X}) \rightarrow N_{n}$ for all $n$. Then the above argument shows that $\gamma \in G$. But $\pi_{1}(\widetilde{X})$ is a free group with a finite number of generators. In particular, it is residually torsion free nilpotent, meaning that $G=\{1\}$. This shows that $\widetilde{A}_{1 n}: S^{1} \rightarrow \widetilde{X}$ is contractible.

As we mentioned above the further proof repeats word-for-word the proof given in $(\mathrm{A})$.

Proof of Corollary 2.17. For the basic facts from the Algebraic Geometry see e.g. the book of Shafarevich $[\mathrm{Sh}]$.

In what follows $\mathbb{C}^{*}:=\mathbb{C} \backslash\{0\}$. If the coefficients $a_{1}, \ldots, a_{n}$ in (2.8) are trigonometric polynomials the map $A_{n}: S^{1} \rightarrow \mathbb{C}^{n}$ can be extended to a holomorphic map $\widetilde{A}_{n}: \mathbb{C}^{*} \rightarrow \mathbb{C}^{n}$ defined by Laurent polynomials. If all components of $A_{n}$ are holomorphic polynomials then the statement of the corollary is trivially fulfilled. Thus we may assume without loss of generality that $\widetilde{A}_{n}$ cannot be extended to $\mathbb{C}$.

Let $\mathbb{C P}^{n}$ denote the complex projective space. Then $\mathbb{C}^{n}=\mathbb{C P}^{n} \backslash H$, where $H$ is the hyperplane at infinity. Now, Zariski closure $\bar{X}$ of the image $X=\widetilde{A}_{n}\left(\mathbb{C}^{*}\right)$ is a (possibly singular) rational curve and $X=\bar{X} \backslash H \subset \mathbb{C}^{n}$ is a closed algebraic subvariety. Further, if the monodromy $\widetilde{\rho}_{n}$ of (2.8) is trivial, then from Corollary 2.15 it follows that $A_{n}: S^{1} \rightarrow X$ is contractible to a point. Let $\nu: \mathbb{C P}^{1} \rightarrow \bar{X}$ be the normalization of $\bar{X}$ and $\widetilde{X}=\nu^{-1}(X) \subset \mathbb{C P}^{1}$ be the normalization of $X$. Then there is an algebraic covering map $\widetilde{A}_{1 n}: \mathbb{C}^{*} \rightarrow \widetilde{X}$ such that $\widetilde{A}_{n}=\nu \circ \widetilde{A}_{1 n}$. In particular, $\widetilde{A}_{1 n}: S^{1} \rightarrow \widetilde{X}$ is contractible to a point. But since $\widetilde{A}_{1 n}: \mathbb{C}^{*} \rightarrow \widetilde{X}$ is a finite 
proper surjective map, the image of the homomorphism $\left(\widetilde{A}_{1 n}\right)_{*}: \pi_{1}\left(\mathbb{C}^{*}\right) \rightarrow \pi_{1}(\widetilde{X})$ is a subgroup of a finite index in $\pi_{1}(\widetilde{X})$. Now the contractibility of $\widetilde{A}_{1 n}: S^{1} \rightarrow \widetilde{X}$ implies that $\pi_{1}(\widetilde{X})=\{1\}$. Thus $X \cong \mathbb{C} \subset \mathbb{C P}^{1}$. Since both maps $\nu: \widetilde{X} \rightarrow \mathbb{C}^{n}$ and $\widetilde{A}_{1 n}: \mathbb{C}^{*} \rightarrow \mathbb{C}$ are algebraic, the latter implies that there are polynomials $p_{1}, \ldots, p_{n} \in \mathbb{C}[z]$ and a Laurent polynomial $q$ such that $\nu(z)=\left(p_{1}(z), \ldots, p_{n}(z)\right)$, $z \in \mathbb{C}$, and $\widetilde{A}_{1 n}(z)=q(z), z \in \mathbb{C}^{*}$. Thus we have $\widetilde{a}_{i}(x)=p_{i}(q(x)), x \in S^{1}, 1 \leq i \leq n$ as required.

Conversely, the above factorization of $A_{n}$ implies that the monodromy $\widetilde{\rho}_{n}$ of the corresponding equation (2.8) is trivial. This can be shown exactly as in the proof of part (A) of Corollary 2.15 and is based on the fact that $\widetilde{X}=\mathbb{C}$ is contratcible to a point.

\section{Proofs of Theorems 2.18, 2.20 and Corollaries 2.21 and 2.22.}

Proof of Theorem 2.18. According to the hypothesis any curve $\Gamma_{k} \subset \mathbb{C}^{k}, k \geq n$, is piecewise smooth. Let $S_{k}$ be the finite set consisting of singularities of $\Gamma_{k}$ and critical values of $A_{k}$. Without loss of generality we may assume that each $S_{k}$ is not empty. By $p_{k}: \Gamma_{k+1} \rightarrow \Gamma_{k}$ we denote the map induced by the projection $\mathbb{C}^{k+1} \rightarrow \mathbb{C}^{k}$ to the first $k$ coordinates. Then $p_{k}: \Gamma_{k+1} \rightarrow \Gamma_{k}, k \geq n$, is a finite surjective map. It is clear that $S_{k+1} \subset p_{k}^{-1}\left(S_{k}\right)$. Then from finiteness it follows that there is a number $K \geq n$ such that for all $k \geq K$, all finite sets $A_{k}^{-1}\left(S_{k}\right) \subset S^{1}$ coincide with a finite set, say, $Y \subset S^{1}$. Then $S^{1} \backslash Y$ is the disjoint union of a finite number of open intervals $I_{1}, \ldots, I_{l}$ such that each $I_{s}$ is diffeomorphic by $A_{k}$ to one of the smooth connected components of $\Gamma_{k} \backslash S_{k}$. Moreover, we have $p_{k}^{-1}\left(S_{k}\right)=S_{k+1}$ and $p_{k}: S_{k+1} \rightarrow S_{k}$ is a bijection. Based on this and using finiteness of each $p_{k}$ we can find a number $N \geq K$ such that for each $k \geq N$ we obtain that $p_{k}: \Gamma_{k+1} \rightarrow \Gamma_{k}$ is a homeomorphism (in fact, it is even diffeomorphism outside sets $S_{k+1}$ and $S_{k}$ ).

Suppose now that $\widehat{\Gamma}_{k}=\Gamma_{k}$ for any $k$, and that the monodromy $\widetilde{\rho}_{N}$ of equation (2.8) is trivial. Then according to Corollary 2.10 and Theorem 2.12 this is equivalent to the contractibility of $A_{N}: S^{1} \rightarrow \Gamma_{N}$ to a point. But it follows from above that $p_{N} \circ p_{N+1} \circ \cdots \circ p_{k}: \Gamma_{k} \rightarrow \Gamma_{N}$ is a homeomorphism for any $k \geq N$. Moreover, $A_{N}=p_{N} \circ p_{N+1} \circ \cdots \circ p_{k} \circ A_{k}$. This implies that $A_{k}: S^{1} \rightarrow \Gamma_{k}, k \geq N$, is contractible to a point. Then from Corollary 2.10 and Theorem 2.12 we obtain that the monodromy $\widetilde{\rho}_{k}$ is trivial for any $k \geq N$. In particular, according to Proposition 2.7 , the monodromy $\widetilde{\rho}$ of $(2.3)$ is trivial.

The proof of the theorem is complete.

Proof of Theorem 2.20. According to Proposition 2.6 the monodromy of the equation $F^{\prime}(x)=\left(\sum_{i=1}^{k} b_{i}^{\prime}(x) t^{i} X_{1} X_{2}^{i-1}\right) \cdot F(x)$ is trivial if and only if

$$
\int \cdots \int_{0 \leq s_{1} \leq \cdots \leq s_{k} \leq 2 \pi} b_{i_{k}}^{\prime}\left(s_{k}\right) \cdots b_{i_{1}}^{\prime}\left(s_{1}\right) d s_{k} \cdots d s_{1}
$$

is zero for any positive integers $i_{1}, \ldots, i_{k}$ and any $k$. Since each $a_{i}$ is the uniform limit of functions of the form $\sum_{j=1}^{k} p_{j i}\left(b_{1}, \ldots, b_{k}\right) \cdot b_{j}^{\prime}$ where $p_{j i} \in \mathbb{C}\left[z_{1}, \ldots, z_{k}\right]$ are 
holomorphic polynomials, from the Ree formula (see Section 4.3 in the proof of Theorem 2.9) it follows directly that each iterated integral

$$
\int \cdots \int_{0 \leq s_{1} \leq \cdots \leq s_{l} \leq 2 \pi} a_{i_{l}}\left(s_{l}\right) \cdots a_{i_{1}}\left(s_{1}\right) d s_{l} \cdots d s_{1}
$$

is the limit of integrals of the form (8.1). This implies that all integrals in (8.2) are zeros. Then Proposition 2.6 implies that the monodromy $\widetilde{\rho}$ of the corresponding equation (2.3) is trivial.

Proof of Corollary 2.21. Passing to polar coordinates we obtain the equation

$$
\frac{d r}{d \phi}=\frac{P}{1+Q} r \quad \text { where } \quad P(r, \phi)=\frac{\partial A(r, \phi)}{\partial \phi}, \quad Q(r, \phi)=B(r, \phi) .
$$

Let us write $H(r, \phi)=h(\phi) r^{k}$ where $h$ is a trigonometric polynomial of degree $k$. Then we have $P(r, \phi)=\sum_{i=1}^{\infty} a_{i} h^{i-1}(\phi) h^{\prime}(\phi) r^{k i}, Q(r, \phi)=\sum_{i=0}^{\infty} b_{i} h^{i}(\phi) r^{k i}$, where $a_{i}, b_{i} \in \mathbb{C}$. Now, if we expand the right-hand side of the above differential equation as a series in $r$, then each coefficient of this series will be of the form $(p \circ h) \cdot h^{\prime}$ where $p \in \mathbb{C}[z]$. In particular, the first integrals of these coefficients satisfy the hypotheses of Corollary 2.17. From this corollary it follows that the required vector field determines a center.

Proof of Corollary 2.22. Passing to polar coordinates we obtain an equation

$$
\frac{d r}{d \phi}=\frac{P}{1+Q} r \quad \text { where } \quad P(r,-\phi)=-P(r, \phi), \quad Q(r,-\phi)=Q(r, \phi) .
$$

In particular $P(r, \phi)=\sum_{j=1}^{\infty} p_{j}(\phi) r^{j}$ where $p_{j}$ are odd trigonometric polynomials, and $Q(r, \phi)=\sum_{j=0}^{\infty} q_{j}(\phi) r^{j}$ where $q_{j}$ are even trigonometric polynomials. Since each $p_{j}$ has the Fourier series expansion by functions $\sin (n \phi), n=1,2, \ldots$, the first integrals $\widetilde{p}_{j}(\phi)=\int_{0}^{\phi} p_{j}(s) d s$ of each $p_{j}$ are even trigonometric polynomials. Thus $\widetilde{p}_{j}$ and $q_{j}$ are polynomials in $\cos \phi$. Now the required result follows from Corollary 2.17 .

\section{Proofs of Results of Section 3.1.}

In this section we prove Propositions 3.1, 3.2, 3.3 and Theorem 3.4.

Proof of Proposition 3.1. Let us consider equation (2.3) related to $a \in X$ introduced in Section 2.2. By $\omega_{a}(x):=\sum_{i=1}^{\infty} a_{i}(x) t^{i} X_{1} X_{2}^{i-1}$ we denote the coefficient of $(2.3)$ and by $\widetilde{\rho}_{a}: \mathbb{Z} \rightarrow G\left(X_{1}, X_{2}\right)[[t]]$ the corresponding monodromy. Let $F_{a}$ : $\mathbb{R} \rightarrow G\left(X_{1}, X_{2}\right)[[t]]$ be the fundamental solution of (2.3) (see Section 2.3). Then $\tilde{\rho}_{a}(n):=F_{a}(2 \pi n)=F_{a}(2 \pi)^{n}, n \in \mathbb{Z}$. By the definition for $a * b, a, b \in X$, we have

$$
F_{a * b}^{\prime}(x):=\left\{\begin{array}{ccc}
2 \omega_{a}(2 x) \cdot F_{a * b}(x) & \text { if } & 0<x \leq \pi \\
2 \omega_{b}(2 x-2 \pi) \cdot F_{a * b}(x) & \text { if } & \pi<x \leq 2 \pi
\end{array}\right.
$$

From here we have

$$
F_{a * b}(x)=\left\{\begin{array}{cll}
F_{a}(2 x) & \text { if } & 0<x \leq \pi \\
F_{b}(2 x-2 \pi) \cdot F_{a}(2 \pi) & \text { if } & \pi<x \leq 2 \pi
\end{array}\right.
$$


In particular,

$$
\widetilde{\rho}_{a * b}(1):=F_{a * b}(2 \pi)=F_{b}(2 \pi) \cdot F_{a}(2 \pi)=\widetilde{\rho}_{b}(1) \cdot \widetilde{\rho}_{a}(1) .
$$

Similarly, for $b^{-1} \in X$ we obtain that

$$
\widetilde{\rho}_{b^{-1}}(1)=\left(\widetilde{\rho}_{b}(1)\right)^{-1}
$$

Suppose now that $a, b \in X$ and $a \sim b$. This means that $a * b^{-1} \in \mathcal{U}$ or, equivalently, that $\widetilde{\rho}_{a * b^{-1}}(1)=1$. Then the above formulae imply that $a \sim b$ if and only if

$$
\widetilde{\rho}_{a}(1)=\widetilde{\rho}_{b}(1)
$$

Now, the fact that $\sim$ is an equivalence relation on $X$ follows easily from (9.3).

The proof of the proposition is complete.

Proof of Proposition 3.2. The proof follows straightforwardly from equations (9.1), (9.2) and (9.3). We leave the details to the reader.

Proof of Proposition 3.3. Suppose $a, b \in X$ and $a \sim b$. We need to show that $I(a)=I(b)$ for any iterated integral $I$. Clearly, it suffices to prove the statement for the basic iterated integrals (see Section 2.1 for the definition).

As before, suppose $F_{a}$ and $F_{b}$ are the fundamental solutions of equations (2.3) corresponding to $a$ and $b$, respectively. It follows from the formula (2.5) that

$$
F_{a}(2 \pi)=\sum_{i=0}^{\infty} q_{i a}\left(2 \pi, X_{1}, X_{2}\right), \quad F_{b}(2 \pi)=\sum_{i=0}^{\infty} q_{i b}\left(2 \pi, X_{1}, X_{2}\right),
$$

where $q_{0 a}=q_{0 b}=I$ and others $q_{i a}, q_{i b}$ are polynomials in $R_{1}, R_{2}, \ldots$ of degree $i$. Moreover, iterated integrals $I_{i_{1}, \ldots, i_{k}}(a)$ and $I_{i_{1}, \ldots, i_{k}}(b)$ with $\sum_{s=1}^{k} i_{s}=i$ are coefficients before the monomial $\left(X_{1} X_{2}^{i_{k}-1}\right) \cdots\left(X_{1} X_{2}^{i_{1}-1}\right)$ in $q_{i a}$ and $q_{i b}$, respectively. Since, by the hypothesis, $F_{a}(2 \pi)=F_{b}(2 \pi)$, Lemma 5.1 then implies that $I_{i_{1}, \ldots, i_{k}}(a)=I_{i_{1}, \ldots, i_{k}}(b)$ for all possible integers $i_{1}, \ldots, i_{k}, k$.

The proposition is proved.

Proof of Theorem 3.4. (1) Suppose, to the contrary, that there are $g_{1}, g_{2} \in G(X)$ such that $g_{1} \neq g_{2}$ and $\widehat{I}\left(g_{1}\right)=\widehat{I}\left(g_{2}\right)$ for every $\widehat{I} \in \mathcal{I}(G(X))$. Let $\tilde{g}_{1}, \tilde{g}_{2} \in X$ be such that $\left[\tilde{g}_{1}\right]=g_{1},\left[\tilde{g}_{2}\right]=g_{2}$. Then by definition we have $I\left(\tilde{g}_{1}\right)=I\left(\tilde{g}_{2}\right)$ for any iterated integral $I$. Then the arguments used in the proof of Proposition 3.3 show that $\tilde{\rho}_{\tilde{g}_{1}}(1)=\tilde{\rho}_{\tilde{g}_{2}}(1)$. This implies that $\tilde{g}_{1} \sim \tilde{g}_{2}$ and so $g_{1}=g_{2}$. This contradiction proves (1).

(2) First we will show that $G(X)$ equipped with the topology $\tau$ is a topological group. (Note that $G(X)$ is a Hausdorff space by the definition of $\tau$.) This will be done by showing that operations of multiplication $\cdot: G(X) \times G(X) \rightarrow G(X)$ and of taking the inverse ${ }^{-1}: G(X) \rightarrow G(X)$ are continuous maps.

Let $(g, h) \in G(X) \times G(X)$ and $U$ be an open neighbourhood of $g h \in G(X)$ of the form

$$
U=\left\{s \in G(X): \max _{1 \leq i \leq k}\left|\widehat{I}_{i}(s)-\widehat{I}_{i}(g h)\right|<\epsilon\right\}
$$


where $I_{1}, \ldots, I_{k}$ are basic iterated integrals, and $0<\epsilon<1$. We recall the following property of iterated integrals (see e.g. [Ha, Proposition 2.9]). For any $g_{1}, g_{2} \in G(X)$,

$$
\widehat{I}_{i_{1}, \ldots, i_{k}}\left(g_{1} g_{2}\right)=\widehat{I}_{i_{1}, \ldots, i_{k}}\left(g_{1}\right)+\sum_{j=1}^{k-1} \widehat{I}_{i_{j+1}, \ldots, i_{k}}\left(g_{1}\right) \cdot \widehat{I}_{i_{1}, \ldots, i_{j}}\left(g_{2}\right)+\widehat{I}_{i_{1}, \ldots, i_{k}}\left(g_{2}\right) \text {. }
$$

Thus without loss of generality we may assume that $\widehat{I}_{l}\left(g_{1} g_{2}\right)=\sum_{j=1}^{t_{l}} R_{l j}\left(g_{1}\right) \cdot S_{l j}\left(g_{2}\right)$, $1 \leq l \leq k$, where $R_{l j}$ and $S_{l j}$ are basic iterated integrals on $G(X)$. We set

$$
M_{1}=\max _{j, l}\left|R_{j l}(g)\right|, \quad M_{2}=\max _{j, l}\left|S_{j l}(h)\right|, \quad M=M_{1}+M_{2}+1, \quad t=\max _{l} t_{l} .
$$

Let us consider open neighbourhoods $U_{1}$ and $U_{2}$ of $g$ and $h$ defined by

$$
\begin{aligned}
& U_{1}=\left\{s_{1} \in G(X): \max _{l, j}\left|R_{l j}(g)-R_{l j}\left(s_{1}\right)\right|<\frac{\epsilon}{M t}\right\}, \\
& U_{2}=\left\{s_{2} \in G(X): \max _{l, j}\left|S_{l j}(h)-S_{l j}\left(s_{2}\right)\right|<\frac{\epsilon}{M t}\right\} .
\end{aligned}
$$

Here $R_{l j}$ and $S_{l j}$ are from the previous formulae. Then for $s_{1} s_{2}$ belonging to the image of $U_{1} \times U_{2}$ under multiplication we have (for $1 \leq l \leq k$ ),

$$
\begin{aligned}
\mid \widehat{I}_{l}(g h)-\widehat{I}_{l}\left(s_{1} s_{2} \mid \leq\right. & \sum_{j=1}^{t_{l}}\left(\left|R_{l j}(g)-R_{l j}\left(s_{1}\right)\right| \cdot\left|S_{l j}(h)\right|+\left|R_{l j}\left(s_{1}\right)\right| \cdot\left|S_{l j}(h)-S_{l j}\left(s_{2}\right)\right|\right) \leq \\
& M_{2} t_{l}\left(\frac{\epsilon}{M t}\right)+\left(M_{1}+\frac{\epsilon}{M t}\right) t_{l}\left(\frac{\epsilon}{M t}\right)<\epsilon .
\end{aligned}
$$

This shows that the image of $U_{1} \times U_{2}$ is containing in $U$. Therefore we established that multiplication : $G(X) \times G(X) \rightarrow G(X)$ is a continuous map.

The continuity of ${ }^{-1}: G(X) \rightarrow G(X)$ follows directly from the definition of the topology $\tau$ and from the formula (see e.g. [Ha, Proposition 2.12])

$$
\widehat{I}_{i_{1}, \ldots, i_{k}}\left(g^{-1}\right)=(-1)^{k} \widehat{I}_{i_{1}, \ldots, i_{k}}(g), \quad g \in G(X) .
$$

Also, the fact that $G(X)$ is separable follows from (3), because every compact metric space is separable and therefore the union of at most countable number of compact metric spaces is separable, as well.

(3) Let us prove that $G(X)$ is metrizable.

By definition the set $\left\{\widehat{I}_{i_{1}, \ldots, i_{k}}\right\}$ of basic iterated integrals is countable. Let us arrange the elements of this set in a sequence $\left\{J_{n}\right\}$ of distinct elements. Now we define

$$
d(g, h)=\sum_{i=1}^{\infty} \frac{2^{-i}\left|J_{i}(g)-J_{i}(h)\right|}{1+\left|J_{i}(g)-J_{i}(h)\right|}, \quad g, h \in G(X) .
$$

It is easy to see that $d$ is a metric in $G(X)$. Let us prove that $d$ is compatible with the topology $\tau$ on $G(X)$. Let

$$
B_{r}(g):=\{h \in G(X): d(h, g)<r\}, \quad r>0,
$$


be a ball centered at $g$ of radius $r$. Since by definition for every fixed $g$ the function $d(g, \cdot)$ is continuous on $(G(X), \tau)$, every ball $B_{r}(g)$ is an open subset of $G(X)$. Suppose

$$
U=\left\{h \in G(X): \max _{1 \leq i \leq k}\left|J_{l_{i}}(g)-J_{l_{i}}(h)\right|<\epsilon\right\}, \quad 0<\epsilon<1,
$$

is an open neighbourhood of $g$. Let $N:=\max _{1 \leq i \leq k} l_{i}$. We set $r:=\frac{\epsilon}{2^{N+1}}$. It is easy to see that if $h \in B_{r}(g)$ then

$$
\frac{2^{-l_{i}}\left|J_{l_{i}}(g)-J_{l_{i}}(h)\right|}{1+\left|J_{l_{i}}(g)-J_{l_{i}}(h)\right|}<r \quad \text { for } \quad 1 \leq i \leq k .
$$

This is equivalent to the inequalities

$$
\left|J_{l_{i}}(g)-J_{l_{i}}(h)\right|<\frac{2^{l_{i}} r}{1-2^{l_{i}} r}<\frac{\epsilon / 2}{1-\epsilon / 2}<\epsilon, \quad 1 \leq i \leq k .
$$

Thus $B_{r}(g) \subset U$. This shows that $d$ is compatible with $\tau$.

Remark 9.1 One can show that the metric space $(G(X), d)$ is not complete.

Prove now that $G(X)$ is the union of an increasing sequence of compact sets.

Given a natural number $n$, let $V_{n} \subset X$ consist of elements $a=\left(a_{1}, a_{2}, \ldots\right)$ such that

$$
\sup _{x \in S^{1}}\left|a_{i}(x)\right| \leq n^{i}, \quad i=1,2, \ldots .
$$

By $K_{n}$ we denote the image of $V_{n}$ in $G(X)$. Clearly, $X=\cup_{n \geq 1} V_{n}$ and $G(X)=\cup_{n \geq 1} K_{n}$. Next, let us prove that $K_{n}$ is compact.

Recall that $X_{i}:=L^{\infty}\left(S^{1}\right)$ is the space of all coefficients $a_{i}$ from equation (1.1). We consider every $X_{i}$ in the weak-star topology. According to the Banach-Alaoglu theorem the set

$$
V_{n i}:=\left\{f \in X_{i}: \sup _{x \in S^{1}}|f(x)| \leq n^{i}\right\}
$$

is weak-star compact. Also, by Tychonoff's theorem, $V_{n}=\prod_{i \geq 1} V_{n i} \subset X$ is compact in the product topology. Since $K_{n}$ is a metric space, in order to prove that $K_{n}$ is compact it suffices to show that every infinite sequence $\left\{x_{k}\right\} \subset K_{n}$ has a limit point in $K_{n}$.

Given $\left\{x_{k}\right\} \subset K_{n}$, let $\left\{y_{k}\right\} \subset V_{n}$ be such that $\left[y_{k}\right]=x_{k}$ for every $k$. Since $V_{n}$ is compact, without loss of generality we may assume that $\lim _{k \rightarrow \infty} y_{k}=y \in V_{n}$ (here $V_{n}$ is equipped with the above product topology). Let us prove that $\lim _{k \rightarrow \infty} x_{k}=[y]$. It is easy to see that the latter is equivalent to the fact that

$$
\lim _{k \rightarrow \infty} I_{i_{1}, \ldots, i_{l}}\left(x_{k}\right)=I_{i_{1}, \ldots, i_{l}}(y)
$$

for every basic iterated integral $I_{i_{1}, \ldots, i_{l}}$. Let $x_{k}=\left(x_{1 k}, x_{2 k}, \ldots\right)$ and $y=\left(y_{1}, y_{2}, \ldots\right)$ where $x_{i k}, y_{i} \in V_{n i}$ for $i=1,2, \ldots$. Then

$$
I_{i_{1}, \ldots, i_{l}}\left(x_{k}\right)=\int \cdots \int_{0 \leq s_{1} \leq \cdots \leq s_{l} \leq 2 \pi} x_{i_{l} k}\left(s_{l}\right) \cdots x_{i_{1} k}\left(s_{1}\right) d s_{l} \cdots d s_{1} .
$$

Our proof is based on the following result. 
Lemma 9.2 Let $\left\{f_{k}\right\},\left\{g_{k}\right\} \subset L^{\infty}([0,2 \pi])$ be uniformly bounded sequences convergent in the weak-star topology to $f, g \in L^{\infty}([0,2 \pi])$, respectively. Let

$$
\tilde{g}_{k}(x)=\int_{0}^{x} g_{k}(s) d s \quad \text { and } \quad \tilde{g}(x)=\int_{0}^{x} g(s) d s, \quad 0 \leq x \leq 2 \pi .
$$

Then the sequence $\left\{f_{k} \tilde{g}_{k}\right\}$ converges in the weak-star topology of $L^{\infty}([0,2 \pi])$ to $f \tilde{g}$.

Proof. According to the hypothesis, there exists a constant $M<\infty$ such that for any $k$,

$$
\sup _{x \in[0,2 \pi]} \max \left\{\left|f_{k}(x)\right|,\left|g_{k}(x)\right|\right\}<M .
$$

From here we obtain that $\tilde{g}_{k}$ converges uniformly on $[0,2 \pi]$ to $\tilde{g}$, and that for any $k$,

$$
\sup _{x \in[0,2 \pi]} \max \left\{\left|\tilde{g}_{k}(x)\right|,|\tilde{g}(x)|\right\} \leq 2 \pi M .
$$

The latter inequality is obvious. Let us check the first statement. Given $\epsilon>0$ choose points $0=t_{1}<t_{2}<\ldots<t_{r}=2 \pi$ from $[0,2 \pi]$ such that $t_{i+1}-t_{i}<\frac{\epsilon}{3 M}$ for any $i$. Since $\left\{g_{k}\right\}$ converges in the weak-star topology to $g$, for any $x \in[0,2 \pi]$ we obtain that

$$
\tilde{g}_{k}(x)=\int_{0}^{2 \pi} g_{k}(s) \chi_{[0, x]}(s) d s \rightarrow \int_{0}^{2 \pi} g(s) \chi_{[0, x]}(s) d s=\tilde{g}(x) \quad \text { as } \quad k \rightarrow \infty .
$$

Here $\chi_{[0, x]}$ is the characteristic function of $[0, x]$. In particular, there exists a natural number $P$ such that for any $p \geq P$ we have

$$
\max _{1 \leq i \leq r}\left|\tilde{g}_{p}\left(t_{i}\right)-\tilde{g}\left(t_{i}\right)\right|<\epsilon / 3 .
$$

Next, for an $x \in[0,2 \pi]$ there is a $t_{i}$ such that $\left|x-t_{i}\right|<\frac{\epsilon}{3 M}$. Thus for $p \geq P$ we have

$$
\begin{gathered}
\left|\tilde{g}_{p}(x)-\tilde{g}(x)\right| \leq\left|\tilde{g}(x)-\tilde{g}\left(t_{i}\right)\right|+\left|\tilde{g}\left(t_{i}\right)-\tilde{g}_{p}\left(t_{i}\right)\right|+\left|\tilde{g}_{p}\left(t_{i}\right)-\tilde{g}_{p}(x)\right|< \\
M \cdot\left(\frac{\epsilon}{3 M}\right)+\frac{\epsilon}{3}+M \cdot\left(\frac{\epsilon}{3 M}\right)=\epsilon .
\end{gathered}
$$

This shows that $\left\{\tilde{g}_{k}\right\}$ converges uniformly on $[0,2 \pi]$ to $\tilde{g}$.

Now, given $h \in L^{1}([0,2 \pi])$ we have

$$
\begin{gathered}
\left|\int_{0}^{2 \pi}\left(f_{k}(s) \tilde{g}_{k}(s)-f(s) \tilde{g}(s)\right) \overline{h(s)} d s\right| \leq \int_{0}^{2 \pi}\left|f_{k}(s)\right| \cdot\left(\left|\tilde{g}_{k}(s)-\tilde{g}(s)\right|\right) \cdot|h(s)| d s+ \\
\left|\int_{0}^{2 \pi}\left(f_{k}(s)-f(s)\right) \tilde{g}(s) \overline{h(s)} d s\right| \leq M \cdot \sup _{s \in[0,2 \pi]}\left|\tilde{g}_{k}(s)-\tilde{g}(s)\right| \cdot \int_{0}^{2 \pi}|h(s)| d s+ \\
\left|\int_{0}^{2 \pi}\left(f_{k}(s)-f(s)\right) \tilde{g}(s) \overline{h(s)} d s\right| .
\end{gathered}
$$


The last two terms in this inequality tend to 0 as $k$ tends to $\infty$. For the first term it follows from the uniform convergence of $\left\{\tilde{g}_{k}\right\}$ to $\tilde{g}$, whereas for the second one because $\overline{\tilde{g}} h \in L^{1}([0,2 \pi])$ and $\left\{f_{k}\right\}$ converges in the weak-star topology to $f$. Thus

$$
\lim _{k \rightarrow \infty} \int_{0}^{2 \pi} f_{k}(s) \tilde{g}_{k}(s) \overline{h(s)} d s=\int_{0}^{2 \pi} f(s) \tilde{g}(s) \overline{h(s)} d s \quad \text { for any } \quad h \in L^{1}([0,2 \pi]) .
$$

This is equivalent to the fact that $f_{k} \tilde{g}_{k}$ converges in the weak-star topology to $f \tilde{g}$.

The proof of the lemma is complete.

Now the statement $\lim _{k \rightarrow \infty} I_{i_{1}, \ldots, i_{l}}\left(x_{k}\right)=I_{i_{1}, \ldots, i_{l}}(y)$ follows by induction on $l$ from Lemma 9.2 if we use the fact that every $\left\{x_{i_{r} k}\right\}_{k \geq 1}$ converges in the weak-star topology of $L^{\infty}([0,2 \pi])$ to $y_{i_{r}}, 1 \leq r \leq l$. We leave the details to the reader.

Therefore we have $\lim _{k \rightarrow \infty} x_{k}=[y]$. According to the preceding discussion this shows that $K_{n}$ is compact, and completes the proof of (3).

(4) Let us prove first that $G(X)$ is contractible to $1 \in G(X)$. To this end for any $t \in \mathbb{R}_{+}$and $a=\left(a_{1}, a_{2}, \ldots,\right) \in X$ we define

$$
t a:=\left(t a_{1}, t a_{2}, \ldots\right) .
$$

Clearly, ta satisfies inequalities (1.5) and so it belongs to $X$. Also, it is easy to see that if $a \sim b$ then $t a \sim t b$. Now we define a map $\Phi:[0,1] \times G(X) \rightarrow G(X)$ by the formula

$$
\Phi(t,[a]):=[t a] .
$$

(Recall that $[a]$ stands for the image of $a \in X$ in $G(X)$.) Let us prove that $\Phi$ is a continuous map. Since $[0,1] \times G(X)$ and $G$ are metrizable, it suffices to check that if a sequence $\left\{\left(t_{k}, g_{k}\right)\right\} \subset[0,1] \times G(X)$ converges to $(t, g) \in G(X)$, then $\left\{\Phi\left(t_{k}, g_{k}\right)\right\}$ converges to $\Phi(t, g)$. In turn, the latter is equivalent to the fact that

$$
\lim _{k \rightarrow \infty} I_{i_{1}, \ldots, i_{l}}\left(t_{k} a_{k}\right)=I_{i_{1}, \ldots, i_{l}}(t a)
$$

for every basic iterated integral $I_{i_{1}, \ldots, i_{l}}$. Here $a_{k}, a \in X$ are such that $\left[a_{k}\right]=g_{k}$ and $[a]=g$. Now, by the definition we have

$$
I_{i_{1}, \ldots, i_{l}}\left(t_{k} a_{k}\right)=t_{k}^{l} I_{i_{1}, \ldots, i_{l}}\left(a_{k}\right) \quad \text { and } \quad I_{i_{1}, \ldots, i_{l}}(t a)=t^{l} I_{i_{1}, \ldots, i_{l}}(a) .
$$

According to our hypothesis $t_{k}^{l} \rightarrow t^{l}$ and $I_{i_{1}, \ldots, i_{l}}\left(a_{k}\right) \rightarrow I_{i_{1}, \ldots, i_{l}}(a)$ as $k \rightarrow \infty$, which implies the required result.

Further, observe that $\Phi(1, \cdot)=i d$ and $\Phi(0, \cdot)$ maps $G(X)$ to $\{1\}$. Thus $\Phi$ determines the required contraction.

Since $G(X)$ is a topological group, to prove that $G(X)$ is arcwise connected it suffices to join every $g \in G(X)$ by a continuous path with $1 \in G(X)$. But then such a path is given by the formula

$$
\gamma(t):=\Phi(t, g), \quad t \in[0,1] .
$$

Let us prove now that $G(X)$ is locally simply and arcwise connected. As before, it suffices to prove that every open neighbourhood of $1 \in G(X)$ of the form

$$
U=\left\{g \in G(X): \max _{1 \leq i \leq l}\left|\widehat{I}_{i}(g)\right|<\epsilon\right\}, \quad \epsilon>0,
$$


where $I_{1}, \ldots, I_{l}$ are non-constant basic iterated integrals, is contractible inside $U$ to 1. Now for every $i, 1 \leq i \leq l$, there is a natural number $s_{i}$ such that $I_{i}(t a)=t^{s_{i}} I_{i}(a)$ for every $(t, a) \in[0,1] \times X$. In particular, for $g \in U$ we have

$$
\max _{1 \leq i \leq l}\left|\widehat{I}_{i}(\Phi(t, g))\right|=\max _{1 \leq i \leq l} t^{s_{i}}\left|\widehat{I}_{i}(g)\right|<\epsilon \cdot \max _{1 \leq i \leq l} t^{s_{i}} \leq \epsilon .
$$

This shows that $\Phi$ maps $[0,1] \times U$ into $U$, and so $U$ is contractible inside $U$ to 1 .

The proof of (4) is complete.

(5) Let us prove that $G(X)$ is residually torsion free nilpotent. Let us consider a subset $H \subset G\left(X_{1}, X_{2}\right)[[t]]$ (see Section 2.2 for the definition) consisting of series of the form

$$
s=\sum_{i=0}^{\infty} p_{i}\left(X_{1}, X_{2}\right) t^{i}
$$

where $p_{0}=I$ and $p_{i}$ are homogeneous polynomials of degree $i$ in free non-commutative variables $X_{1}$ and $X_{2}$ with complex coefficients. It is easy to see that $H$ is a group with respect to the multiplication in $G\left(X_{1}, X_{2}\right)[[t]]$. Further, according to equations (9.1), (9.2) and (9.3) there exists a natural injective homomorphism $\phi: G(X) \rightarrow H$ defined by the formula

$$
\phi([a]):=\widetilde{\rho}_{a}(1), \quad a \in X,
$$

where 1 is the unit in $\mathbb{Z}$ and $\widetilde{\rho}_{a}$ is the monodromy of equation (2.3) corresponding to $a \in X$. Thus it suffices to prove that $H$ is a residually torsion free nilpotent group.

First, observe that the number of monomials of degree $i$ in variables $X_{1}, X_{2}$ is finite. Thus the vector space (over $\mathbb{C}$ ) of homogeneous polynomials of degree $i$ in $X_{1}, X_{2}$ with complex coefficients is finite-dimensional. Let us consider a subset $H_{n} \subset H$ consisting of series of the form

$$
s=I+\sum_{i=n+1}^{\infty} p_{i}\left(X_{1}, X_{2}\right) t^{i}
$$

Lemma 9.3 $H_{n}$ is a normal subgroup of $H$.

Proof. It easy to see that $s_{1} s_{2} \in H_{n}$ for any $s_{1}, s_{2} \in H_{n}$. Thus $H_{n} \subset H$ is a subgroup.

Let $s=I+\sum_{i=1}^{\infty} p_{i}\left(X_{1}, X_{2}\right) t^{i} \in H$ and $s^{-1}=I+\sum_{i=1}^{\infty} \widetilde{p}_{i}\left(X_{1}, X_{2}\right) t^{i}$. From the identity $s s^{-1}=I$ it follows easily that in the product

$$
l_{n}=I+\sum_{j=1}^{\infty} q_{j}\left(X_{1}, X_{2}\right) t^{j}:=\left(I+\sum_{i=1}^{n} p_{i}\left(X_{1}, X_{2}\right) t^{i}\right) \cdot\left(I+\sum_{i=1}^{n} \widetilde{p}_{i}\left(X_{1}, X_{2}\right) t^{i}\right)
$$

$q_{1}=\cdots=q_{n}=0$. Further observe that for an $h=I+\sum_{i=n+1}^{\infty} h_{i}\left(X_{1}, X_{2}\right) t^{i} \in H_{n}$, there exist homogeneous polynomials $\widetilde{h}_{i}\left(X_{1}, X_{2}\right)$ of degree $i, n+1 \leq i<\infty$, in $X_{1}, X_{2}$ such that

$$
s h s^{-1}=l_{n}+\sum_{i=n+1}^{\infty} \widetilde{h}_{i}\left(X_{1}, X_{2}\right) t^{i} .
$$

This shows that $s h s^{-1} \in H_{n}$ and completes the proof of the lemma. 
Now, the quotient group $Q_{n}:=H / H_{n}$ can be identified with the set of the elements

$$
s=I+\sum_{i=1}^{n} p_{i}\left(X_{1}, X_{2}\right) \hat{t}^{i}
$$

where $p_{i}$ are homogeneous polynomials of degree $i$ in $X_{1}, X_{2}$ with complex coefficients, and

$$
\hat{t}^{i} \cdot \hat{t}^{j}=\left\{\begin{array}{cll}
\hat{t}^{i+j} & \text { if } & i+j \leq n \\
0 & \text { if } & i+j>n
\end{array}\right.
$$

Here the multiplication of the coefficients $p_{i}$ of such elements is the same as in the algebra of non-commutative polynomials.

A straightforward computation shows that $Q_{n}$ is a nilpotent group. Moreover, $Q_{n}$ is finite-dimensional and has a natural structure of a complex algebraic group. In particular, $Q_{n}$ admits a faithful finite-dimensional unipotent representation $t_{n}: Q_{n} \rightarrow G L_{N}(\mathbb{C})$. (For the basic results about algebraic groups see e.g. [OV, Chapter 3].)

Let $\pi_{n}: H \rightarrow Q_{n}$ be the quotient homomorphism. Observe that the family $\left\{t_{n} \circ \pi_{n}\right\}$ of finite-dimensional unipotent representations separates elements of $H$. This shows that $H$ is residually torsion free nilpotent and completes the proof of the theorem.

\section{Proofs of Results of Section 3.2.}

In this section we prove Propositions 3.5, 3.8, and Theorems 3.6, 3.7, 3.9 and 3.10. Proof of Proposition 3.5. Let $G_{c}[[r]]$ be the set of complex power series $f(r)=$ $r+\sum_{k=1}^{\infty} d_{k}(f) r^{k+1}$ each convergent in some open neighbourhood of $0 \in \mathbb{C}$. First, we will show that $G_{c}[[r]]$ is a group with respect to the operation of composition.

Suppose $f_{1}, f_{2} \in G_{c}[[r]]$. Then by definition, there exists an open disk $D_{i}$ centered at 0 such that $f_{i}$ is a holomorphic function on $D_{i}, i=1,2$. In particular, there exists an open disk $D_{1}^{\prime} \subset D_{1}$ centered at 0 such that $f_{1}\left(D_{1}^{\prime}\right) \subset D_{2}$. Therefore $f_{2} \circ f_{1}$ : $D_{1}^{\prime} \rightarrow \mathbb{C}$ is a holomorphic function such that $\left(f_{2} \circ f_{1}\right)(0)=0$ and $\left(f_{2} \circ f_{1}\right)^{\prime}(0)=1$. This implies that $f_{2} \circ f_{1}$ has a Taylor series expansion in some open disk centered at 0 . Thus $f_{2} \circ f_{1} \in G_{c}[[r]]$. Next, for $f \in G_{c}[[r]]$, by the inverse function theorem we obtain that $f^{-1}$ exists, is holomorphic on an open disk containing 0 , and $f^{-1}(0)=0$, $\left(f^{-1}\right)^{\prime}(0)=1$. Hence, $f^{-1} \in G_{c}[[r]]$.

Further, recall that the topology $\tau^{\prime}$ on $G_{c}[[r]]$ is the weakest topology in which all the functions $d_{k}: G_{c}[[r]] \rightarrow \mathbb{C}$ are continuous. It is obvious that the family $\left\{d_{k}\right\}_{k \geq 1}$ separates points on $G_{c}[[r]]$. Also, since the set of these functions is countable, $G_{c}[[r]]$ is metrizable (cf. the proof of Theorem 3.4 (3) for a similar argument). Thus in order to prove that $\left(G_{c}[[r]], \tau^{\prime}\right)$ is a topological group it suffices to check that

(1) if $\left\{f_{i}\right\},\left\{g_{i}\right\} \subset G_{c}[[r]]$ converge to $f, g \in G_{c}[[r]]$, respectively, then $\left\{f_{i} \circ g_{i}\right\}$ converges to $f \circ g$.

(2) if $\left\{f_{i}\right\} \subset G_{c}[[r]]$ converges to $f \in G_{c}[[r]]$, then $\left\{f_{i}^{-1}\right\}$ converges to $f^{-1}$. 
In turn, $\lim _{i \rightarrow \infty} h_{i}=h$ in $\tau^{\prime}$ is equivalent to the fact that $\lim _{i \rightarrow \infty} d_{k}\left(h_{i}\right)=d_{k}(h)$ for any $k$. Next, note that $d_{k}\left(f_{i} \circ g_{i}\right)=P_{k}\left(d_{1}\left(f_{i}\right), \ldots, d_{k}\left(f_{i}\right), d_{1}\left(g_{i}\right), \ldots, d_{k}\left(g_{i}\right)\right)$ and $d_{k}\left(f_{i}^{-1}\right)=Q_{k}\left(d_{1}\left(f_{i}\right), \ldots, d_{k}\left(f_{i}\right)\right)$ for any $i$, where $P_{k} \in \mathbb{C}\left[z_{1}, \ldots, z_{2 k}\right]$ and $Q_{k} \in$ $\mathbb{C}\left[z_{1}, \ldots, z_{k}\right]$ are complex holomorphic polynomials. This implies (1) and (2).

Further, clear that the subset of polynomials with rational coefficients is dense in $\left(G_{c}[[r]], \tau^{\prime}\right)$. Therefore $G_{c}[[r]]$ is separable.

Now, the contraction map can be defined by the formula

$$
F(t, f)(r):=\frac{f(t r)}{t}, \quad t \in[0,1], f \in G_{c}[[r]] .
$$

It is easy to check that $F:[0,1] \times G_{c}[[r]] \rightarrow G_{c}[[r]]$ is continuous, and $F(1, \cdot)=i d$, $F(0, \cdot)=1$, where 1 is the unit of $G_{c}[[r]]$, i.e. the function $f(r)=r$.

Now, the facts that $G_{c}[[r]]$ is arcwise connected and locally simply and arcwise connected can be obtained exactly in the same way as similar statements for $G(X)$ (cf. the proof of Theorem $3.4(4)$ ).

Finally, let us show that $G_{c}[[r]]$ is residually torsion free nilpotent.

To this end, let us introduce a subset $G_{n} \subset G_{c}[[r]]$ consisting of series of the form

$$
f(r)=r+\sum_{k=n+1}^{\infty} d_{k} r^{k}
$$

Then using the arguments similar to those of Lemma 9.3 one can show that $G_{n}$ is a normal subgroup of $G_{c}[[r]]$. Further, the quotient group $L_{n}:=G_{c}[[r]] / G_{n}$ can be identified with the set of elements

$$
f(\widehat{r})=\widehat{r}+\sum_{k=1}^{n} d_{k} \widehat{r}^{k}
$$

such that

$$
\widehat{r}^{i} \circ \widehat{r}^{j}=\left\{\begin{array}{ccc}
\widehat{r}^{i j} & \text { if } \quad i \cdot j \leq n \\
0 & \text { if } & i \cdot j>n
\end{array}\right.
$$

Then $L_{n}$ admits a faithful unipotent representation $q_{n}: L_{n} \rightarrow G L_{N}(\mathbb{C})$ because, as it is easy to see, $L_{n}$ has a structure of a finite-dimensional nilpotent algebraic group. If $\pi_{n}: G_{c}[[r]] \rightarrow L_{n}$ is the quotient homomorphism, then, by definition, the family $\left\{q_{n} \circ \pi_{n}\right\}$ separates elements of $G_{c}[[r]]$. Thus $G_{c}[[r]]$ is residually torsion free nilpotent.

The proof of the proposition is complete.

Proof of Theorem 3.6. We should check that $P(a * b)=P(b) \circ P(a)$, for $a, b \in X$. As before, let $v(x ; r ; c), x \in[0,2 \pi]$, be the Lipschitz solution of equation (1.1) corresponding to $c \in X$ with the initial value $v(0 ; r ; c)=r$. By the definition of $a * b$ we have

$$
v(x ; r ; a * b)=\left\{\begin{array}{ccc}
v(2 x ; r ; a) & \text { if } \quad 0<x \leq \pi \\
v(2 x-2 \pi ; v(2 \pi ; r ; a) ; b) & \text { if } \quad \pi<x \leq 2 \pi
\end{array}\right.
$$

Thus by the definition of the first return map $P$ (see Section 2.1) we obtain that

$$
P(a * b)(r):=v(2 \pi ; r ; a * b)=v(2 \pi ; v(2 \pi ; r ; a) ; b)=P(b)(P(a)(r)) .
$$


This completes the proof of the theorem.

Proof of Theorem 3.7. The map $\widehat{P}: G(X) \rightarrow G_{c}[[r]]$ is given by the formula

$$
\widehat{P}([a])=P(a), \quad a \in X .
$$

It is correctly defined because $P(a)(r) \equiv r$ for any $a \in \mathcal{U}$. Also, from Theorem 3.6 it follows that $\widehat{P}$ is a homomorphism of groups. Let us prove that $\widehat{P}$ is continuous.

Suppose $\left\{g_{n}\right\} \subset G(X)$ converges to $g \in G(X)$. We must show that $\left\{\widehat{P}\left(g_{n}\right)\right\}$ converges to $\widehat{P}(g)$. (This is equivalent to the continuity of $\widehat{P}$ because $G(X)$ and $G_{c}[[r]]$ are metric spaces.) Also, as it was mentioned before, the latter is equivalent to the following statement

$\lim _{n \rightarrow \infty} d_{k}\left(\widehat{P}\left(g_{n}\right)\right)=d_{k}(\widehat{P}(g))$ for any $k$, provided that $\lim _{n \rightarrow \infty} \widehat{I}\left(g_{n}\right)=\widehat{I}(g)$ for any iterated integral $\widehat{I}$ on $G(X)$.

Now, according to Theorem 2.2, $d_{k}\left(\widehat{P}\left(g_{n}\right)\right)=c_{k}\left(g_{n}^{\prime}\right)$, where $g_{n}^{\prime} \in X$ is such that $\left[g_{n}^{\prime}\right]=g_{n}$, and $c_{k}$ is an iterated integral on $X$. Thus the above statement is true. This shows that $\widehat{P}: G(X) \rightarrow G_{c}[[r]]$ is a homomorphism of topological groups.

Let us prove that $\widehat{P}$ is a surjection.

Given $f(r)=r+\sum_{k=1}^{\infty} d_{k} r^{k+1} \in G_{c}[[r]]$ we define $a(f)=\left(a_{1}, a_{2}, \ldots\right)$ by the identity of formal power series

$$
\sum_{i=1}^{\infty} a_{i}(x) t^{i+1}=\frac{\sum_{k=1}^{\infty}\left(d_{k} / 2 \pi\right) t^{k+1}}{1+\sum_{k=1}^{\infty}(k+1) d_{k}(1-x / 2 \pi) t^{k}} \quad \text { for } \quad x \in(0,2 \pi]
$$

and further extended by periodicity. By our hypothesis on $f$ it follows that the expression on the right-hand side of $(10.1)$ is a bounded, continuous in $x \in(0,2 \pi)$ function, holomorphic in $t$ for $t$ varying in a small open disk centered at 0 . This and the Cauchy inequalities for derivatives of a holomorphic function imply that $a(f) \in X$. Let $v(x ; r ; a(f))$ be the Lipschitz solution of equation (1.1) corresponding to $a(f) \in X$ with the initial value $v(0 ; r ; a(f))=r$. Then from equation (10.1) it follows that for any sufficiently small $r$,

$$
v(x ; r ; a(f))+\sum_{k=1}^{\infty} d_{k}(1-x / 2 \pi)[v(x ; r ; a(f))]^{k+1}=f(r), \quad 0 \leq x \leq 2 \pi .
$$

Since $P(a(f))(r):=v(2 \pi ; r ; a(f))$, the latter implies that

$$
P(a(f))(r)=f(r) .
$$

So $\widehat{P}$ is a surjection.

It is clear that $P(a)(r) \equiv r$ if and only if $a$ belongs to the center set $\mathcal{C} \subset X$. This shows that $\widehat{\mathcal{C}}:=\operatorname{Ker}(\widehat{P})$ coincides with the image of $\mathcal{C}$ in $G(X)$. Then $\widehat{\mathcal{C}}$ is a normal subgroup of $G(X)$. It is closed because $\widehat{P}$ is continuous. Since there are examples of non-universal centers (see e.g. Remark 2.24), $\widehat{\mathcal{C}}$ is non-trivial. Now, the contraction of $\widehat{\mathcal{C}}$ is defined as follows.

For $a=\left(a_{1}, a_{2}, \ldots\right) \in X$ we define $a_{t}=\left(a_{1 t}, a_{2 t}, \ldots\right) \in X$ by the formula

$$
a_{i t}=t^{i} a_{i}, \quad 1 \leq i<\infty .
$$


Then

$$
I_{i_{1}, \ldots, i_{k}}\left(a_{t}\right)=t^{i_{1}+\ldots+i_{k}} I_{i_{1}, \ldots, i_{k}}(a)
$$

for every basic iterated integral $I_{i_{1}, \ldots, i_{k}}$. This implies that if $a \sim b$ then $a_{t} \sim b_{t}$. In particular, we can define a map $F:[0,1] \times G(X) \rightarrow G(X)$ by the formula

$$
F(t,[a]):=\left[a_{t}\right], \quad a \in X .
$$

Now from Theorem 2.2 it follows that

$$
c_{n}\left(a_{t}\right)=t^{n} c_{n}(a), \quad a \in X, \quad n=1,2, \ldots .
$$

This shows that $F$ maps $\widehat{\mathcal{C}}$ to itself. Moreover, $F(1, \cdot)=i d, F(0, \cdot)=1$, and, according to (10.2), $F$ is continuous. Thus $F:[0,1] \times \widehat{\mathcal{C}} \rightarrow \widehat{\mathcal{C}}$ is the required contraction. Now the statements that $\widehat{\mathcal{C}}$ is arcwise connected, locally simply and arcwise connected can be obtained in a way similar to that of the proof of Theorem 3.4 (4). We leave the details to the reader.

The proof of Theorem 3.7 is complete.

Proof of Proposition 3.8. By definition, $\widehat{c}_{n}([a])=c_{n}(a), a \in X$, where $c_{n}$, $1 \leq n<\infty$, are the Taylor coefficients of the first return map $P$ (see Theorem 2.2). In particular, we have

$$
\widehat{P}(s)(r)=r+\sum_{n=1}^{\infty} \widehat{c}_{n}(s) r^{n+1}, \quad s \in G(X),
$$

and the series converges absolutely for sufficiently small $r$. Suppose $g \in \widehat{\mathcal{C}}$. Then, according to Theorem 3.6, for every $h \in G(X)$ we have $\widehat{P}(h g)=\widehat{P}(g h)=\widehat{P}(h)$. This is equivalent to the identities

$$
r+\sum_{n=1}^{\infty} \widehat{c}_{n}(h g) r^{n+1}=r+\sum_{n=1}^{\infty} \widehat{c}_{n}(g h) r^{n+1}=r+\sum_{n=1}^{\infty} \widehat{c}_{n}(h) r^{n+1} .
$$

Thus

$$
\widehat{c}_{n}(h g)=\widehat{c}_{n}(g h)=\widehat{c}_{n}(h), \quad 1 \leq n<\infty .
$$

Therefore every $\widehat{c}_{n}, 1 \leq n<\infty$, is constant on fibres of the quotient homomorphism $\pi: G(X) \rightarrow Q(X):=G(X) / \widehat{\mathcal{C}}$, and hence determines a function $\bar{c}_{n}: Q(X) \rightarrow \mathbb{C}$ such that $\bar{c}_{n} \circ \pi=\widehat{c}_{n}$.

The proof of the proposition is complete.

Proof of Theorem 3.9. Let $\tau^{\prime \prime}$ be the weakest topology on $Q(X)$ in which all functions $\bar{c}_{n}$ are continuous. According to formula (10.3) and Proposition 3.8, the homomorphism $\widehat{P}$ determines a homomorphism $\bar{P}: Q(X) \rightarrow G_{c}[[r]]$ defined by $\widehat{P}=\bar{P} \circ \pi$. In particular, we have

$$
\bar{P}(q)(r)=r+\sum_{n=1}^{\infty} \bar{c}_{n}(q) r^{n+1}, \quad q \in Q(X),
$$

and the series converges absolutely for sufficiently small $r$. By definition, $\bar{P}$ : $Q(X) \rightarrow G_{c}[[r]]$ is a group isomorphism. According to (10.4), $\bar{P}$ is continuous, 
because the functions $\bar{c}_{n}$ are continuous on $Q(X)$. Let us prove now that $\bar{P}^{-1}$ is also continuous. Since the set of functions $\left\{\bar{c}_{n}\right\}$ is countable, $Q(X)$ is metrizable (cf. the arguments used in the proof of Theorem $3.4(3))$. In particular, it suffices to prove the following statement

Suppose $\left\{f_{k}\right\} \subset G_{c}[[r]]$ is such that $\lim _{k \rightarrow \infty} f_{k}=f \in G_{c}[[r]]$ in the topology $\tau^{\prime}$ on $G_{c}[[r]]$. Then the sequence $\left\{h_{k}:=\bar{P}^{-1}\left(f_{k}\right)\right\} \subset Q(X)$ converges in $\tau^{\prime \prime}$ to $h:=\bar{P}^{-1}(f)$.

Let $f_{k}(r)=r+\sum_{n=1}^{\infty} d_{n}\left(f_{k}\right) r^{n+1}, 1 \leq k<\infty$, and $f(r)=r+\sum_{n=1}^{\infty} d_{n}(f) r^{n+1}$. Then identity (10.4) implies that $\bar{c}_{n}\left(h_{k}\right)=d_{n}\left(f_{k}\right)$, and $\bar{c}_{n}(h)=d_{n}(h)$ for any $k, n$. Now by the hypothesis we have $\lim _{k \rightarrow \infty} d_{n}\left(f_{k}\right)=d_{n}(f)$ for any $n$. This and the above identity imply that $\lim _{k \rightarrow \infty} \bar{c}_{n}\left(h_{k}\right)=\bar{c}_{n}(h)$ for any $n$. The latter is equivalent to $\lim _{k \rightarrow \infty} h_{k}=h$ in $\tau^{\prime \prime}$. Thus $\bar{P}$ is an isomorphism of topological groups.

The proof of the theorem is complete.

Proof of Theorem 3.10. Let $a(f) \in X, f \in G_{c}[[r]]$, be defined by equation (10.1). By $T: G_{c}[[r]] \rightarrow G(X)$ we denote the map $f \mapsto[a(f)]$. In the proof of Theorem 3.7 we established that $(\widehat{P} \circ T)(f)=f$ for any $f \in G_{c}[[r]]$. Let us prove now that $T$ is continuous. In fact, from (10.1) it follows that

$$
a_{i}(x)=p_{i}\left(x, d_{1}(f), \ldots, d_{i}(f)\right), \quad 1 \leq i<\infty, \quad x \in(0,2 \pi],
$$

where every $p_{i} \in \mathbb{C}\left[z_{1}, \ldots, z_{i+1}\right]$ is a holomorphic polynomial. In particular, by definition, for any basic iterated integral $I_{i_{1}, \ldots, i_{k}}$ we have

$$
I_{i_{1}, \ldots, i_{k}}(a(f))=\widetilde{p}_{i_{1}, \ldots, i_{k}}\left(d_{1}(f), \ldots, d_{l}(f)\right)
$$

where $l=\max \left\{i_{1}, \ldots, i_{k}\right\}$, and $\widetilde{p}_{i_{1}, \ldots, i_{k}} \in \mathbb{C}\left[z_{1}, \ldots, z_{l}\right]$ is a holomorphic polynomial. Now, this implies that

if $\lim _{s \rightarrow \infty} f_{s}=f$ on $G_{c}[[r]]$, then $\lim _{s \rightarrow \infty} \widehat{I}_{i_{1}, \ldots, i_{k}}\left(T\left(f_{s}\right)\right)=\widehat{I}_{i_{1}, \ldots, i_{k}}(T(f))$ for every basic iterated integral $\widehat{I}_{i_{1}, \ldots, i_{k}}$ on $G(X)$.

This is equivalent to the fact that $\lim _{s \rightarrow \infty} T\left(f_{s}\right)=T(f)$ on $(G(X), \tau)$. Thus $T$ is a continuous embedding.

Further, let us define $\widetilde{T}: G_{c}[[r]] \times \widehat{\mathcal{C}} \rightarrow G(X)$ by the formula

$$
\widetilde{T}(f, g)=T(f) \cdot g, \quad f \in G_{c}[[r]], g \in \widehat{\mathcal{C}} .
$$

Since $T$ and $\cdot$ are continuous maps, $\widetilde{T}$ is continuous. Let us show that $\widetilde{T}$ is a bijection. Indeed, if we have $T\left(f_{1}\right) \cdot g_{1}=T\left(f_{2}\right) \cdot g_{2}$ then

$$
f_{1}=\widehat{P}\left(T\left(f_{1}\right) \cdot g_{1}\right)=\widehat{P}\left(T\left(f_{2}\right) \cdot g_{2}\right)=f_{2} .
$$

This implies that $\left(f_{1}, g_{1}\right)=\left(f_{2}, g_{2}\right)$. Thus $\widetilde{T}$ is an injection. Now for any $h \in G(X)$ we obviously have

$$
h=\widetilde{T}(f, g), \quad \text { where } \quad f=\widehat{P}(h) \quad \text { and } \quad g=(T \circ \widehat{P})\left(h^{-1}\right) \cdot h .
$$

Thus $\widetilde{T}$ is a surjection. Also, the inverse to $\widetilde{T}$ is defined by the formula

$$
\widetilde{T}^{-1}(h):=\left(\widehat{P}(h),(T \circ \widehat{P})\left(h^{-1}\right) \cdot h\right), \quad h \in G(X) .
$$

It is clearly a continuous map because $\widehat{P}, T, \cdot$ and $^{-1}$ are continuous.

Thus $\tilde{T}$ is a homeomorphism. This completes the proof of the theorem. 


\section{Proofs of Results of Section 3.3.}

In this section we prove Propositions 3.11, 3.14, 3.15, and Theorems 3.12, 3.13, 3.16. Proof of Proposition 3.11. The statements

(1) the family of iterated integrals separates points on $G_{s}(X)$ and $G_{a}(X)$;

(2) $G_{s}(X)$ and $G_{a}(X)$ are separable topological groups in the relative topologies induced by $\tau$;

(3) $G_{s}(X)$ and $G_{a}(X)$ are residually torsion free nilpotent groups

follow directly from the fact that $G_{s}(X)$ and $G_{a}(X)$ are subgroups of $G(X)$ and $G(X)$ satisfies similar properties.

Let us prove that $G_{s}(X)$ and $G_{a}(X)$ are contractible to a point, arcwise connected, locally simply and arcwise connected.

We recall that the map $\Phi:[0,1] \times G(X) \rightarrow G(X)$ is defined by the formula

$$
\Phi(t,[a])=[t a], \quad a \in X
$$

where $t a=\left(t a_{1}, t a_{2}, \ldots\right)$ for $a=\left(a_{1}, a_{2}, \ldots\right)$ (see the proof of Theorem $\left.3.4(4)\right)$. Observe that if $a \in X_{a}$ then $t a \in X_{a}$ for any $t$. Since by the definition $X_{a} \subset X_{s}$, from the above we obtain that $\Phi$ maps $[0,1] \times G_{s}(X)$ into $G_{s}(X)$ and $[0,1] \times G_{a}(X)$ into $G_{a}(X)$. This and the properties of $\Phi$ described in the proof of Theorem $3.4(4)$ imply the required statements.

The proof of the proposition is complete.

Proof of Theorem 3.12. From formula (10.1) it follows that for any $f \in G_{c}[[r]]$ the element $a(f) \in X_{a} \subset X_{s}$. Thus the map $T, f \mapsto[a(f)]$, maps $G_{c}[[r]]$ into $G_{a}(X) \subset G_{s}(X)$. Since $(\widehat{P} \circ T)(f)=f$, the latter implies that

$$
\left.\widehat{P}\right|_{G_{s}(X)}: G_{s}(X) \rightarrow G_{c}[[r]] \text { and }\left.\widehat{P}\right|_{G_{a}(X)}: G_{a}(X) \rightarrow G_{c}[[r]]
$$

are surjective homomorphisms of topological groups. By definition their kernels coincide with $\widehat{\mathcal{C}}_{s}$ and $\widehat{\mathcal{C}}_{a}$, respectively. Also, the maps $\widetilde{T}_{s}: G_{c}[[r]] \times \widehat{\mathcal{C}}_{s} \rightarrow G_{s}(X)$, $\widetilde{T}_{s}(f, g)=T(f) \cdot g$, and $\widetilde{T}_{a}: G_{c}[[r]] \times \widehat{\mathcal{C}}_{a} \rightarrow G_{a}(X), \widetilde{T}_{a}(f, g)=T(f) \cdot g$ are homeomorphisms (this can be shown exactly in the same way as for $\widetilde{T}$ in the proof of Theorem 3.10). From here it follows that $G_{s}(X) / \widehat{\mathcal{C}}_{s}$ and $G_{a}(X) / \widehat{\mathcal{C}}_{a}$ are isomorphic to $G_{c}[[r]]$ which, in turn, is isomorphic to $Q(X)$.

The proof of the theorem is complete.

Proof of Theorem 3.13. The proof repeats word-for-word proofs of Proposition 3.11 and Theorem 3.12. We leave the details to the reader.

Proof of Proposition 3.14. From property (9.4) for iterated integrals on $G(X)$ it follows that it suffices to prove the following statement

The image in $G(X)$ of every $a=\left(a_{1}, a_{2}, \ldots\right) \in X_{\mathbb{F}^{\prime}}(b)$ is defined over $\mathbb{F}^{\prime}$.

Recall that every $a_{k}$ is a linear function in $\hat{b}_{i_{1}}, \ldots, \hat{b}_{i_{s}}$ with coefficients from $\mathbb{F}^{\prime}$. Here $\hat{b}=\left(\hat{b}_{1}, \hat{b}_{2}, \ldots\right) \in X$ is such that 
(a) its components run over the set of all possible monomials determined by elements of a sequence $b=\left\{b_{i}\right\} \subset L^{\infty}\left(S^{1}\right)$ satisfying $\sup _{i, S^{1}}\left|b_{i}\right| \leq 1$;

(b) $[\hat{b}] \in G(X)$ is defined over the field $\mathbb{F}\left(\subseteq \mathbb{F}^{\prime}\right)$.

Now, the definition of $a_{k}$ implies that every $I_{j_{1}, \ldots, j_{p}}(a)$ is a linear combination with coefficients from $\mathbb{F}^{\prime}$ of values of certain basic iterated integrals computed at $\hat{b}$. In particular, $\widehat{I}_{j_{1}, \ldots, j_{p}}(a) \in \mathbb{F}^{\prime}$.

This completes the proof of the proposition.

Proof of Proposition 3.15. In what follows by $\left[X_{\mathbb{F}^{\prime}}(b)\right]^{-1}$ we denote the set of elements $a \in X$ such that $a^{-1} \in X_{\mathbb{F}^{\prime}}(b)$.

Let $\sigma: \mathbb{F}^{\prime} \rightarrow \mathbb{F}^{\prime}$ be a field automorphism over $\mathbb{F}$. For any $a \in X_{\mathbb{F}^{\prime}}(b) \subset X$ we define $\sigma(a), \sigma\left(a^{-1}\right) \in X_{\mathbb{F}^{\prime}}(b)$ as follows.

Suppose $a_{i}=\sum_{j=1}^{s(i)} c_{i j} \hat{b}_{i j}$ where $c_{i j} \in \mathbb{F}^{\prime}$ and $\hat{b}_{i j}$ are components of $\hat{b} \in X$, $1 \leq j \leq s(i), i \in \mathbb{N}$. Then

$$
\begin{gathered}
\sigma(a):=\left(\sigma\left(a_{1}\right), \sigma\left(a_{2}\right), \ldots\right), \quad \sigma\left(a^{-1}\right):=\left(\sigma\left(a_{1}^{-1}\right), \sigma\left(a_{2}^{-1}\right), \ldots\right) \quad \text { where } \\
\sigma\left(a_{i}\right):=\sum_{j=1}^{s(i)} \sigma\left(c_{i j}\right) \hat{b}_{i j}, \quad \sigma\left(a_{i}^{-1}\right):=\sum_{j=1}^{s(i)} \sigma\left(c_{i j}\right) \hat{b}_{i j}^{-1}, \quad 1 \leq j \leq s(i), i \in \mathbb{N} .
\end{gathered}
$$

In particular, we have $\sigma\left(a^{-1}\right)=\sigma(a)^{-1}$.

Further, define the map $\widetilde{\sigma}: G_{\mathbb{F}^{\prime}}(b) \rightarrow G_{\mathbb{F}^{\prime}}(b)$ by the formula

$$
\tilde{\sigma}\left(\left[a_{1}\right] \cdots\left[a_{s}\right]\right)=\left[\sigma\left(a_{1}\right)\right] \cdots\left[\sigma\left(a_{s}\right)\right], \quad a_{1}, \ldots, a_{s} \in X_{\mathbb{F}^{\prime}}(b) \cup\left[X_{\mathbb{F}^{\prime}}(b)\right]^{-1} .
$$

Let us show that $\widetilde{\sigma}$ is correctly defined.

First, suppose $a, a^{\prime} \in X_{\mathbb{F}^{\prime}}(b) \cup\left[X_{\mathbb{F}^{\prime}}(b)\right]^{-1}$ are such that $a \sim a^{\prime}$. Then for any basic iterated integral we have $I(a)=I\left(a^{\prime}\right)$. Moreover, the definition for the components $a_{i}$ and $a_{i}^{\prime}$ of $a$ and $a^{\prime}$ and the fact that $[\hat{b}]$ is defined over the field $\mathbb{F}$ imply that there exist $c_{1}, \ldots, c_{l} \in \mathbb{F}^{\prime}$ (that coincide with coefficients $c_{i j}, c_{i j}^{\prime}$ in the decompositions of certain $a_{i}$ and $\left.a_{i}^{\prime}\right)$ such that $I(a)=p(a)$ and $I\left(a^{\prime}\right)=q\left(a^{\prime}\right)$ where $p, q \in \mathbb{F}\left[z_{1}, \ldots, z_{l}\right]$ are polynomials with coefficients from $\mathbb{F}$ and $p(a), q\left(a^{\prime}\right)$ are their values at the point $\left(c_{1}, \ldots, c_{l}\right) \in\left(\mathbb{F}^{\prime}\right)^{l}$. Note that for $\sigma(a)$ and $\sigma\left(a^{\prime}\right)$ we have $I(\sigma(a))=p(\sigma(a))$ and $I\left(\sigma\left(a^{\prime}\right)\right)=q\left(\sigma\left(a^{\prime}\right)\right)$ with the same $p$ and $q$, where $p(\sigma(a))$ and $q\left(\sigma\left(a^{\prime}\right)\right)$ are the values of $p$ and $q$ at $\left(\sigma\left(c_{1}\right), \ldots, \sigma\left(c_{l}\right)\right) \in\left(\mathbb{F}^{\prime}\right)^{l}$. Since $\sigma$ is a field automorphism over $\mathbb{F}$,

$$
I(\sigma(a))=p(\sigma(a))=\sigma(p(a))=\sigma\left(q\left(a^{\prime}\right)\right)=q\left(\sigma\left(a^{\prime}\right)\right)=I\left(\sigma\left(a^{\prime}\right)\right) .
$$

This shows that $\sigma(a) \sim \sigma\left(a^{\prime}\right)$. Thus $[\sigma(a)], a \in X_{\mathbb{F}^{\prime}}(b) \cup\left[X_{\mathbb{F}^{\prime}}(b)\right]^{-1}$, depends only on the equivalence class of $a$.

Suppose now $g_{1}, \ldots, g_{s}, h_{1}, \ldots, h_{l} \in G_{\mathbb{F}^{\prime}}(b)$ are such that $g_{1} \cdots g_{s}=h_{1} \cdots h_{l}$, and $g_{i}=\left[g_{i}^{\prime}\right], 1 \leq i \leq s, h_{j}=\left[h_{j}^{\prime}\right], 1 \leq j \leq l$, for some $g_{1}^{\prime}, \ldots, g_{s}^{\prime}, h_{1}^{\prime}, \ldots, h_{l}^{\prime} \in$ $X_{\mathbb{F}^{\prime}}(b) \cup\left[X_{\mathbb{F}^{\prime}}(b)\right]^{-1}$. Then for a basic iterated integral $\widehat{I}$ by formula (9.4) we obtain that

$$
\widehat{I}\left(g_{1} \cdots g_{s}\right)=\sum_{j=1}^{r} R_{j 1}\left(g_{1}\right) \cdots R_{j s}\left(g_{s}\right), \quad \widehat{I}\left(h_{1} \cdots h_{l}\right)=\sum_{n=1}^{t} S_{n 1}\left(h_{1}\right) \cdots S_{n l}\left(h_{l}\right)
$$


where all $R_{j i}$ and $S_{n k}$ are basic iterated integrals on $G(X)$. Also, according to the above argument, $R_{j i}\left(g_{i}\right)=p_{j i}\left(g_{i}\right)$ and $S_{n k}=q_{n k}\left(h_{k}\right)$ where there exists $m \in \mathbb{N}$ such that $p_{j i}, q_{n k} \in \mathbb{F}\left[z_{1}, \ldots, z_{m}\right]$ and $p_{j i}\left(g_{i}\right), q_{n k}\left(h_{k}\right)$ are the values of these polynomials at some $\left(c_{1}, \ldots, c_{m}\right) \in\left(\mathbb{F}^{\prime}\right)^{m}(1 \leq j \leq r, 1 \leq i \leq s, 1 \leq n \leq t, 1 \leq k \leq l)$. In particular, we have (below we retain the notation of the first part of the proof)

$$
\begin{gathered}
\widehat{I}\left(\widetilde{\sigma}\left(g_{1}\right) \cdots \widetilde{\sigma}\left(g_{s}\right)\right)=\sum_{j=1}^{r} R_{j 1}\left(\widetilde{\sigma}\left(g_{1}\right)\right) \cdots R_{j s}\left(\widetilde{\sigma}\left(g_{s}\right)\right)=\sum_{j=1}^{r} \sigma\left(R_{j 1}\left(g_{1}\right)\right) \cdots \sigma\left(R_{j s}\left(g_{s}\right)\right)= \\
\sigma\left(\widehat{I}\left(g_{1} \cdots g_{s}\right)\right)=\sigma\left(\widehat{I}\left(h_{1} \cdots h_{l}\right)\right)=\sum_{n=1}^{l} \sigma\left(R_{n 1}\left(h_{1}\right)\right) \cdots \sigma\left(R_{n l}\left(h_{l}\right)\right)= \\
\sum_{n=1}^{l} R_{n 1}\left(\tilde{\sigma}\left(h_{1}\right)\right) \cdots R_{n l}\left(\widetilde{\sigma}\left(h_{l}\right)\right)=\widehat{I}\left(\tilde{\sigma}\left(h_{1}\right) \cdots \widetilde{\sigma}\left(h_{l}\right)\right) .
\end{gathered}
$$

This shows that $\tilde{\sigma}\left(g_{1}\right) \cdots \tilde{\sigma}\left(g_{s}\right)=\tilde{\sigma}\left(h_{1}\right) \cdots \tilde{\sigma}\left(h_{l}\right)$. Thus $\tilde{\sigma}$ is correctly defined. Clearly, $\tilde{\sigma}(1)=1$. Therefore the definition of $\widetilde{\sigma}$ shows that it is an automorphism of $G_{\mathbb{F}^{\prime}}(X)$. (Here we do not assert that $\widetilde{\sigma}$ is continuous in the topology on $G_{\mathbb{F}^{\prime}}(X)$.)

Let us proof now that $\tilde{\sigma}\left(\widehat{\mathcal{C}}_{\mathbb{F}^{\prime}}(b)\right)=\widehat{\mathcal{C}}_{\mathbb{F}^{\prime}}(b)$.

Suppose $g \in \widehat{\mathcal{C}}_{\mathbb{F}^{\prime}}(b)$. Then $\widehat{c}_{n}(g)=0,1 \leq n<\infty$, where $\widehat{c}_{n}$ are defined in Section 3.2. According to Theorem 2.2 every $\widehat{c}_{n}$ is the sum of basic iterated integrals with integer coefficients. Also, according to the above computation, $\sigma(\widehat{I}(h))=\widehat{I}(\widetilde{\sigma}(h))$, $h \in G_{\mathbb{F}^{\prime}}(b)$, for any basic iterated integral $\widehat{I}$. Therefore for any $n$ we have

$$
\widehat{c}_{n}(\widetilde{\sigma}(g))=\sigma\left(\widehat{c}_{n}(g)\right)=0 .
$$

This equivalent to the fact that $\tilde{\sigma}(g) \in \widehat{\mathcal{C}}_{\mathbb{F}^{\prime}}(b)$.

The proof of the proposition is complete.

Proof of Theorem 3.16. First, let us prove that $[P]:[0,1] \rightarrow G(X)$ is a continuous map. To this end, we must check that $\widehat{I}([P](t))$ is a continuous function on $[0,1]$ for every basic iterated integral $\widehat{I}$. Now, according to formulae $(9.4),(9.5)$ it suffices to prove the statement for $P(t)=c(t)$ where $c(t)=\left(c_{1}(t), c_{2}(t), \ldots\right)$ with $c_{k}(t)$ polynomials in $t$ whose coefficients are elements from $\mathcal{A}_{\mathbb{F}^{\prime}}(b)$. But then a simple computation shows that $\widehat{I}(c(t)) \in \mathbb{C}[t]$. This implies the continuity of $[P]$.

Now let us prove the theorem. According to Proposition 3.14 and formulae (9.4), (9.5), for every function $\widehat{c}_{n}$ (defined in Section 3.2) the function $\widehat{c}_{n}([P](t)) \in \mathbb{F}^{\prime}[t]$. Now, the hypothesis of the theorem implies that $\widehat{c}_{n}([P](\xi))=0$ for every $n$, and there exists $k \in \mathbb{N}$ such that $\widehat{c}_{k}([P](0)) \neq 0$. In particular, this shows that $\widehat{c}_{k}([P])(t)$ is a non-trivial polynomial. Since the coefficients of this polynomial belong to $\mathbb{F}^{\prime}$ and $\widehat{c}_{k}([P](\xi))=0$, the number $\xi$ is algebraic over $\mathbb{F}^{\prime}$.

This completes the proof of the theorem.

\section{References}

[A] H. Alexander, Polynomial approximation and hulls in sets of finite linear measure in $\mathbb{C}^{n}$, Amer. J. Math. 93 (1971), 65-74. 
[AL] M. A. M. Alwash and N. G. Lloyd, Non-autonomous equations related to polynomial two-dimensional systems, Proc. Roy. Soc. Edinburgh Sect. A 105 (1987), 129-152.

[AW] H. Alexander and J. Wermer, Several Complex Variables and Banach Algebras, Springer-Verlag, New York, 1998.

[B] I. Bendixson, Sur les courbes définies par des équations différentielles, Acta Math., 24 (1901), 1-88.

[BFY1] M. Briskin, J.-P. Francoise and Y. Yomdin, Center condition II: Parametric and model center problems, Isr. J. Math. 118, (2000), 61-82.

[BFY2] M. Briskin, J.-P. Francoise and Y. Yomdin, Center condition III: Parametric and model center problems, Isr. J. Math. 118, (2000), 83-108.

[B] L. Bungart, On analytic fibre bundles I. Holomorphic fibre bundles with infinite dimensional fibres, Topology 7 no.1 (1968), 55-68.

[Bl] M. Blinov, Center and composition conditions for Abel equation, Thesis, Weizmann Institute of Science, 2002.

[Che] L. Cherkas, Number of limit cycles of an autonomous second-order system, Differentsial'nye uravneniya 12 (1976), no.5, 944-946.

[F] M. Frommer, Die Integralkurven einer gewöhnlichen Differential-gleichung erster Ordnung in der Umgebung rationaler Unbestimmtheitsstellen, Math. Ann., 99 (1928), 222-272.

[GR] H. Grauert and R. Remmert, Theorie der Steinschen Räume, Springer-Verlag, New York, 1977.

[Gr] H. Grauert, Analytische Faserungen über Holomorph Vollständigen Räumen, Math. Ann. 135 (1958), 263-278.

[H] H. A. Hamm, Zum Homotopietyp Steinscher Räume, J. Reine Angew. Math., 338 (1983), 121-135.

[HL] G. Henkin and J. Leiterer, Theory of functions on complex manifolds, Birkhäuser-Verlag, Basel, 1984.

[Ha] R. M. Hain, The geometry of the mixed Hodge structure on the fundamental group, Proc. of Symposia in Pure Math. 46 (1987), 247-282.

[Hi] F. Hirzebruch, Topological methods in Algebraic Geometry, Springer-Verlag, New York, 1966.

[Hu] S.-T. Hu, Homotopy Theory, Academic Press, New York, 1959.

[I] Yu. Ilyashenko, Centennial history of Hilbert's 16th problem, Bull. Amer. Math. Soc. 39 (2002), no.3, 301-354. 
[K] N. H. Kuiper, The homotopy type of the unitary group of Hilbert space, Topology 3 no.1 (1965), 19-30.

[KN] S. Kobayashi and K. Nomizu, Foundations of differential geometry, Interscience, New York, 1963.

[L] A. M. Lyapunov, The general problem of the stability of motion, Translated by A. T. Fuller from Édouard Daraux's French translation (1907) of the 1892 Russian original, Internat. J. Control 55 (1992), no. 3., 521-790.

[Lo] S. Lojasiewicz, Triangulation of semi-analytic sets, Ann. Scuola Norm. Sup. Pisa, (3) 18 (1964), 449-474.

[Na] R. Narasimhan, Analysis on real and complex manifolds, North Holland, Amsterdam, 1973.

[O] A. L. Onishchik, Certain concepts and applications of non-Abelian cohomology theory, Trudy Moskov. Mat. Obshch. 17 (1967), 45-88.

[OV] A. L. Onishchik and E. B. Vinberg, A seminar on Lie groups and algebraic groups, (Russian), URSS, Moscow, 1995.

[P] H. Poincaré, Sur les courbes définies par une équation différentielle, Oeuvres, t.1, Paris, 1892.

[R] R. Ree, Lie elements and an algebra associated with shuffles, Ann. of Math. (2) 68 (1958), 210-220.

[Ru] W. Rudin, Real and complex analysis, McGraw-Hill, 1987.

[Su] D. Sullivan, Infinitesimal computations in topology, Publ. Math. IHES, 47 (1977), 269-332.

[Sh] I. Shafarevich, Basic algebraic geometry, Springer-Verlag, 1974.

[Si] K. S. Sibirsky, Introduction to the algebraic theory of invariants of differential equations, Tranlsated from the Russian, Nonlinear Science: theory and applications, Manchester University Press, Manchester, 1988.

[Y] Y. Yomdin, Center problem for Abel equation, compositions of functions and moment conditions, preprint (2002), 40 pp. 